\title{
Efficient Access to Orthoquinols and Their [4+2] Cyclodimers via SIBX-Mediated Hydroxylative Phenol Dearomatization
}

Nathalie Lebrasseur, ${ }^{\dagger \dagger}$ Julien Gagnepain ${ }^{\dagger, \dagger}$ Aurélie Ozanne-Beaudenon, ${ }^{\dagger, \ddagger}$ Jean-Michel Léger,${ }^{\S}$ and Stéphane Quideau*,t,

Université de Bordeaux, 'Institut Européen de Chimie et Biologie, 2 rue Robert Escarpit, 33607 Pessac Cedex, France, ${ }^{t}$ Institut des Sciences Moléculaires (CNRS-UMR 5255), 351 cours de la Libération, 33405 Talence Cedex, France, and ${ }^{\S}$ Laboratoire de Pharmacochimie, 146 rue Léo Saignat, 33076 Bordeaux Cedex, France

s.quideau@iecb.u-bordeaux.fr

\section{Supporting Information}

\section{CONTENTS}

Experimental details and characterization data

Influence of an acidic medium on the IBX-mediated HPD of 2,6-dimethylphenol (7a) 
General Experimental Methods. All reactions were run under $\mathrm{N}_{2}$ using stabilized oiodoxybenzoic acid (SIBX), the IBX content of which being $49 \%$ by weight of SIBX. Tetrahydrofuran (THF) was purified by distillation from sodium/benzophenone under $\mathrm{N}_{2}$ prior to use. Dichloromethane $\left(\mathrm{CH}_{2} \mathrm{Cl}_{2}\right)$, ethyl acetate (EtOAc), toluene, acetone and dimethyl sulfoxide (DMSO) were used as received. Evaporations were conducted under reduced pressure at temperatures less than $45^{\circ} \mathrm{C}$ unless otherwise noted. Column chromatography was carried out under positive pressure using 40-63 $\mu \mathrm{m}$ silica gel and the indicated solvents. Further drying of the residues was accomplished under high vacuum, except for volatile products. Melting points are uncorrected. NMR spectra of samples in the indicated solvent were run at 250, 300 or $400 \mathrm{MHz}$. Carbon multiplicities were determined by DEPT-135 or $J$-MOD experiments. Electron impact mass spectra were obtained at $70 \mathrm{eV}$. Low and high resolution electron impact, chemical ionisation and liquid secondary ion mass spectrometry data (EIMS, CIMS, LSIMS, HRMS) were obtained either from the mass spectrometry laboratory at the Institut Européen de Chimie et Biologie, Pessac, France, from the Centre d'Etude Structurale et d'Analyse des Molécules Organiques (CESAMO) de l'Université de Bordeaux, Talence, France, or from the Centre Régional de Mesures Physiques de l'Ouest (CRMPO), Rennes, France.

3,10-Dihydroxy-3,5,8,10-tetramethyltricyclo $\left[6.2 .2 .0^{2,7}\right]$ dodeca-5,11-diene-4,9-dione (9a). The SIBX-mediated HPD of 2,6-dimethylphenol 7a (1.22 g, $10 \mathrm{mmol})$ was run according to the general procedure A. Purification by column chromatography, eluting with hexanes/acetone (3:1), gave dimer 9a $(1.32 \mathrm{~g}, 96 \%)$ as a white solid. Crystallization from a mixture of $\mathrm{CH}_{2} \mathrm{Cl}_{2}$ and hexanes afforded colourless prisms, which were used for X-ray analysis. The resulting crystal structure was identical to that previously reported for 9a. ${ }^{10 \mathrm{~b}} \mathrm{mp} 172{ }^{\circ} \mathrm{C}$ (lit. $\left.{ }^{10 \mathrm{a}} \mathrm{mp} 194-196{ }^{\circ} \mathrm{C}\right)$; IR (NaCl, neat) 3416, 1713, $1670 \mathrm{~cm}^{-1} ;{ }^{1} \mathrm{H}$ NMR $\left(\mathrm{CDCl}_{3}, 300 \mathrm{MHz}\right) \delta 1.24(\mathrm{~s}, 3 \mathrm{H}), 1.32(\mathrm{~s}, 3 \mathrm{H}), 1.43(\mathrm{~s}, 3 \mathrm{H}), 1.85$ (s, 3H), $2.33(\mathrm{~s}, 1 \mathrm{H}), 2.80-2.95(\mathrm{~m}, 1 \mathrm{H}), 3.25(\mathrm{dd}, J=1.7,8.3 \mathrm{~Hz}, 1 \mathrm{H}), 3.38(\mathrm{~d}, J=6.8 \mathrm{~Hz}, 1 \mathrm{H}), 4.00$ $(\mathrm{s}, 1 \mathrm{H}), 5.51(\mathrm{~d}, J=8.1 \mathrm{~Hz}, 1 \mathrm{H}), 6.20-6.35(\mathrm{~m}, 2 \mathrm{H}) ;{ }^{13} \mathrm{C} \mathrm{NMR}\left(\mathrm{CDCl}_{3}, 100 \mathrm{MHz}\right) \delta 214.8,203.0$, 139.3, 135.7, 135.3, 133.2, 73.6, 72.9, 53.7, 44.2, 43.6, 42.7, 31.7, 26.2, 16.3, 15.6; EIMS m/z (rel. intensity) $276\left(\mathrm{M}^{+}, 5\right), 242$ (3), $216(3), 138$ (42), 122 (15), 121 (33), 43 (100).

\section{3,10-Dihydroxy-3,5,7,8,10,11-hexamethyl-tricyclo[6.2.2.0 $\left.{ }^{2,7}\right]$ dodeca-5,11-diene-4,9-dione}

(9b). The SIBX-mediated HPD of 2,4,6-trimethylphenol 7b (1.36 g, $10 \mathrm{mmol})$ was run according to the general procedure A. Purification by column chromatography, eluting with hexanes/acetone (3:1), gave dimer $9 \mathbf{b}(1.42 \mathrm{~g}, 94 \%)$ as a white solid. Crystallization from a mixture of $\mathrm{CH}_{2} \mathrm{Cl}_{2}$ and hexanes afforded colourless prisms, which were used for X-ray analysis. The resulting crystal data were deposited at the Cambridge Crystallographic Data Centre under the number CCDC 640072. 9b: mp 159-162 ${ }^{\circ} \mathrm{C}$ (lit. ${ }^{10 a} \mathrm{mp} 182-183{ }^{\circ} \mathrm{C}$ ); IR ( $\mathrm{NaCl}$, neat) 3482, 2979, 2876, $1686 \mathrm{~cm}^{-1} ;{ }^{1} \mathrm{H}$ NMR 
$\left(\mathrm{CDCl}_{3}, 400 \mathrm{MHz}\right) \delta 1.16(\mathrm{~s}, 3 \mathrm{H}), 1.24(\mathrm{~s}, 6 \mathrm{H}), 1.36(\mathrm{~s}, 3 \mathrm{H}), 1.70(\mathrm{~d}, J=1.7 \mathrm{~Hz}, 3 \mathrm{H}), 1.83(\mathrm{~d}, J=$ $1.5 \mathrm{~Hz}, 3 \mathrm{H}), 2.23$ (bs, 1H), 2.80 (d, $J=2.1 \mathrm{~Hz}, 1 \mathrm{H}), 3.14$ (t, $J=2.1 \mathrm{~Hz}, 1 \mathrm{H}), 3.92(\mathrm{~s}, 1 \mathrm{H}), 5.04$ (s, $1 \mathrm{H}), 6.03(\mathrm{~s}, 1 \mathrm{H}) ;{ }^{13} \mathrm{C} \mathrm{NMR}\left(\mathrm{CDCl}_{3}, 75.5 \mathrm{MHz}\right) \delta 214.0,202.3,145.1,144.9,132.8,127.3,73.6$, 71.9, 57.6, 48.6, 48.5, 45.1, 32.3, 25.0, 23.0, 21.0, 16.1, 12.3; EIMS $m / z$ (rel. intensity) $304\left(\mathrm{M}^{+}, 1\right)$, 152 (18), $136(32), 110(55), 28(71)$.

6-Hydroxy-2,5,6-trimethylcyclohexa-2,4-dienone (8c) and 3,10-Dihydroxy-3,5,6,8,10,12hexamethyl-tricyclo[6.2.2.0 $\left.{ }^{2,7}\right]$ dodeca-5,11-diene-4,9,-dione (9c). The SIBX-mediated HPD of 2,3,6-trimethylphenol 7c (1.36 g, $10 \mathrm{mmol})$ was run according to the general procedure A apart from the fact that a saturated aqueous solution of $\mathrm{Na}_{2} \mathrm{CO}_{3}$ was used instead of an aqueous $1 \mathrm{M}$ solution of $\mathrm{NaOH}$ during the work-up. Purification by column chromatography, eluting with hexanes/acetone from (10:1) to (3:1), afforded orthoquinol $\mathbf{8 \mathbf { c } ^ { 1 3 \mathrm { a } }}(480 \mathrm{mg}, 32 \%)$ as a bright yellow oil and the dimer 9c (471 mg, 31\%) as a colourless solid. 8c: $\mathrm{Rf}=0.6$ [hexanes/acetone (3:1)]; IR ( $\mathrm{NaCl}) 3415,2922$, 1716, $1115 \mathrm{~cm}^{-1} ;{ }^{1} \mathrm{H}$ NMR $\left(\mathrm{CDCl}_{3}, 300 \mathrm{MHz}\right) \delta 1.34(\mathrm{~s}, 3 \mathrm{H}), 1.89(\mathrm{~s}, 3 \mathrm{H}), 1.98(\mathrm{~s}, 3 \mathrm{H}), 5.79(\mathrm{~d}, J=$ $6.0 \mathrm{~Hz}, 1 \mathrm{H}), 6.71(\mathrm{~d}, J=6.0 \mathrm{~Hz}, 1 \mathrm{H}) ;{ }^{13} \mathrm{C} \mathrm{NMR}\left(\mathrm{CDCl}_{3}, 75 \mathrm{MHz}\right) \delta 207.7,152.9,139.5,129.1$, 117.0, 78.8, 29.3, 17.6, 14.8; CIMS $\left(\mathrm{NH}_{3}\right) \mathrm{m} / z$ (rel. intensity) $170\left(\mathrm{MNH}_{4}{ }^{+}, 100\right)$; HRMS (EI) calcd for $\mathrm{C}_{9} \mathrm{H}_{12} \mathrm{O}_{2}$ 152.0837, found 152.0842. Crystallization of 9c from a mixture of $\mathrm{CH}_{2} \mathrm{Cl}_{2}$ and hexanes afforded colourless prisms, which were used for X-ray analysis. The resulting crystal data were deposited at the Cambridge Crystallographic Data Centre under the number CCDC 640073. 9c: mp $180{ }^{\circ} \mathrm{C}$ (lit. $\left.{ }^{13 \mathrm{a}} \mathrm{mp} 191-192{ }^{\circ} \mathrm{C}\right) ; \mathrm{Rf}=0.1$ [hexanes/acetone (3:1)]; IR (NaCl, neat) 3404, 2913, 1640, 1623, 1664, 1377, $1121 \mathrm{~cm}^{-1} ;{ }^{1} \mathrm{H}$ NMR $\left(\mathrm{CDCl}_{3}, 300 \mathrm{MHz}\right) \delta 1.17(\mathrm{~s}, 3 \mathrm{H}), 1.22(\mathrm{~s}, 6 \mathrm{H}), 1.43(\mathrm{~s}, 3 \mathrm{H})$, $1.81(\mathrm{~s}, 3 \mathrm{H}), 1.98(\mathrm{~s}, 3 \mathrm{H}), 2.88(\mathrm{~d}, J=7.8 \mathrm{~Hz}, 1 \mathrm{H}), 2.98(\mathrm{~d}, J=7.8 \mathrm{~Hz}, 1 \mathrm{H}), 3.32(\mathrm{~d}, J=6.1 \mathrm{~Hz}, 1 \mathrm{H})$, $5.96(\mathrm{~d}, J=6.1 \mathrm{~Hz}, 1 \mathrm{H}) ;{ }^{13} \mathrm{C} \mathrm{NMR}\left(\mathrm{CDCl}_{3}, 75 \mathrm{MHz}\right) \delta 213.7,202.8,148.3,136.9,132.3,131.0$, 73.7, 71.9, 56.4, 48.9, 44.1, 41.2, 32.0, 26.2, 23.6, 18.7, 15.1, 13.5; EIMS $\mathrm{m} / z$ (rel. intensity) 304 $\left(\mathrm{M}^{+}, 9\right), 240$ (16), 152 (42), 135 (85), 123 (73), 109 (71), 95 (100). HRMS (EI) calcd for $\mathrm{C}_{18} \mathrm{H}_{24} \mathrm{O}_{4}$ 304.1675, found 304.1671 .

6-Hydroxy-5-methoxy-2,6-dimethylcyclohexa-2,4-dienone (8d) and 3,10-Dihydroxy-6methoxy-3,5,8,10-tetramethyl-tricyclo[6.2.2.0 $\left.{ }^{2,7}\right]$ dodeca-5-ene-4,9,12-trione (9d). The SIBXmediated HPD of 3-methoxy-2,6-dimethylphenol 7d (152 mg, $1 \mathrm{mmol})$ was run according to the general procedure $A$ apart from the fact that a saturated aqueous solution of $\mathrm{Na}_{2} \mathrm{CO}_{3}$ was used instead of an aqueous $1 \mathrm{M}$ solution of $\mathrm{NaOH}$ during the work-up. Purification by column chromatography, eluting with $\mathrm{CH}_{2} \mathrm{Cl}_{2} / \mathrm{MeOH}$ from (100:1) to (50:1), afforded orthoquinol $8 \mathbf{d}$ (70 $\mathrm{mg}, 42 \%)$ as a bright yellow oil and dimer 9d (72 $\mathrm{mg}, 43 \%)$ as a colourless solid. $8 \mathbf{d}$ : $\mathrm{Rf}=0.3$ $\left[\mathrm{CH}_{2} \mathrm{Cl}_{2} / \mathrm{MeOH}(100: 1)\right]$; IR $(\mathrm{NaCl}) 3370,2930,1730,1140 \mathrm{~cm}^{-1} ;{ }^{1} \mathrm{H}$ NMR $\left(\mathrm{CDCl}_{3}, 300 \mathrm{MHz}\right) \delta$ 
$1.46(\mathrm{~s}, 3 \mathrm{H}), 1.88(\mathrm{~d}, J=1.3 \mathrm{~Hz}, 6 \mathrm{H}), 3.43(\mathrm{~s}, 1 \mathrm{H}), 3.76(\mathrm{~s}, 3 \mathrm{H}), 5.13$ (d, $J=7.2 \mathrm{~Hz}, 1 \mathrm{H}), 6.89$ (qd, $J$ $=1.3,7.2 \mathrm{~Hz}, 1 \mathrm{H}) ;{ }^{13} \mathrm{C} \mathrm{NMR}\left(\mathrm{CDCl}_{3}, 75 \mathrm{MHz}\right) \delta 203.2,167.6,141.4,123.9,91.3,76.8,56.1,29.4$, 14.6; CIMS $\left(\mathrm{NH}_{3}\right) \mathrm{m} / z$ (rel. intensity) $186\left(\mathrm{MNH}_{4}^{+}, 100\right)$; HRMS (EI) calcd for $\mathrm{C}_{9} \mathrm{H}_{12} \mathrm{O}_{3} 168.0786$, found 168.0790. 9d: $\mathrm{mp} 153-154{ }^{\circ} \mathrm{C} ; \mathrm{Rf}=0.1$ [hexanes/acetone (3:1)]; IR ( $\mathrm{NaCl}$, neat) 3469, 2952, 1740, 1718, 1664, 1322, 1215, $1042 \mathrm{~cm}^{-1} ;{ }^{1} \mathrm{H}$ NMR $\left(\mathrm{CDCl}_{3}, 300 \mathrm{MHz}\right) \delta 1.19$ (s, 3H), 1.37 (s, 6H), $1.86(\mathrm{~s}, 3 \mathrm{H}), 2.12(\mathrm{~d}, J=21.7 \mathrm{~Hz}, 1 \mathrm{H}), 2.47$ (d, $J=21.7 \mathrm{~Hz}, 1 \mathrm{H}), 2.87$ (s, 1H), 3.10 (s, 1H), 3.20 (s, 1H), 3.77 (s, 3H), 4.11 (br s, $1 \mathrm{H}) ;{ }^{13} \mathrm{C} \mathrm{NMR}\left(\mathrm{CDCl}_{3}, 75 \mathrm{MHz}\right) \delta 212.3,204.8,202.0,168.6,117.4$, 76.5, 72.3, 66.4, 60.0, 45.5, 38.0, 37.7, 31.2, 22.9, 12.5, 10.3; EIMS $m / z$ (rel. intensity) $322\left(\mathrm{M}^{+}, 1\right)$, 277(92), 233 (60), 155 (83), 140 (100); HRMS (EI) calcd for $\mathrm{C}_{15} \mathrm{H}_{19} \mathrm{O}_{5}$ [M $\left.\mathrm{M}^{+}-\mathrm{CHO}\right] 279.1232$, found 279.1235 .

3,10-Dihydroxy-3,6,10,12-tetramethyltricyclo $\left[6.2 .2 .0^{2,7}\right]$ dodeca-5,11-diene-4,9-dione

(9e). The SIBX-mediated HPD of 2,5-dimethylphenol (7e) (1.22 g, $10 \mathrm{mmol})$ was run according to the general procedure B. Purification by column chromatography, eluting with hexanes/acetone (30:1), gave dimer 9e as a brown solid $(730 \mathrm{mg})$, which was further purified by crystallization from a mixture of $\mathrm{CH}_{2} \mathrm{Cl}_{2}$ and hexanes to furnish pure $9 \mathrm{e}(676 \mathrm{mg}, 49 \%)$ as colourless prisms. These prisms were used for X-ray analysis, and the resulting crystal data were deposited at the Cambridge Crystallographic Data Centre under the number CCDC 640074. 9e: mp $189{ }^{\circ} \mathrm{C}\left(\right.$ lit. $^{10 \mathrm{c}} \mathrm{mp} 200-201$ $\left.{ }^{\circ} \mathrm{C}\right) ; \mathrm{R}_{\mathrm{f}}=0.10$ [hexanes/acetone, (3:1)]; IR ( NaCl, neat) 3435, 1729, 1671, 1379, $1159\left(\mathrm{~cm}^{-1}\right) ;{ }^{1} \mathrm{H}$ NMR $\left(\mathrm{CDCl}_{3}, 300 \mathrm{MHz}\right) \delta 1.25(\mathrm{~s}, 3 \mathrm{H}), 1.30(\mathrm{~s}, 3 \mathrm{H}), 1.61(\mathrm{~d}, J=1.3 \mathrm{~Hz}, 3 \mathrm{H}), 2.00(\mathrm{~d}, J=1.0 \mathrm{~Hz}$, $3 \mathrm{H}), 2.28$ (bs, 1H), 3.10-3.20 (m, 3H), 3.32 (dd, $J=1.3,6.8 \mathrm{~Hz}, 1 \mathrm{H}), 4.00$ (bs, 1H), 5.87 (d, $J=6.8$ $\mathrm{Hz}, 1 \mathrm{H}), 6.02(\mathrm{~s}, 1 \mathrm{H}) ;{ }^{13} \mathrm{C} \mathrm{NMR}\left(\mathrm{CDCl}_{3}, 100 \mathrm{MHz}\right) \delta 212.7,201.4,156.3,136.5,128.3,124.9,73.1$, 72.9, 56.9, 44.7, 44.2, 41.1, 32.0, 25.9, 22.4, 21.5; EIMS m/z (rel intensity) $276\left(\mathrm{M}^{+}, 69\right), 216$ (13), 215 (52), 188 (35), 187 (100), 159 (65), 122 (43).

\section{6,12-Di-tert-butyl-3,10-dihydroxy-3,10-dimethyltricyclo $\left[6.2 .2 .0^{2,7}\right]$ dodeca-5,11-diene-4,9-}

dione (9f). The SIBX-mediated HPD of 5-tert-butyl-2-methylphenol $7 \mathbf{f}$ (70 mg, $0.43 \mathrm{mmol}$ ) was run according to the general procedure B. Purification by column chromatography, eluting with hexanes/EtOAc from (4:1) to (1:1), furnished dimer 9 f (30 mg, 39\%) as a colourless solid. ${ }^{9 b}$

3,10-Dihydroxy-6,12-di-isopropyl-3,10-dimethyl-tricyclo $\left[6.2 .2 .0^{2,7}\right]$ dodeca-5,11-diene-4,9dione (9g) and 2,3-dihydroxy-4-iso-propyl-1-methylbenzene (10g). Carcavrol (7g) (1.5 g, 10 mmol) was submitted to the SIBX-mediated HPD reaction according to the general procedure B. Purification by column chromatography, eluting with hexanes/Et $\mathrm{t}_{2} \mathrm{O}(1: 1)$, afforded 2,3-dihydroxy-4iso-propyl-1-methylbenzene $(\mathbf{1 0 g})^{25}$ as a brown oil $(398 \mathrm{mg}, 24 \%)$ and dimer $9 \mathrm{~g}$ as a yellow solid $(930 \mathrm{mg})$. This solid was crystallized from a mixture of $\mathrm{CH}_{2} \mathrm{Cl}_{2}$ and hexanes to give pure $\mathbf{9 g}$ as white 
needles (795 mg, 48\%). 10g: $\mathrm{R}_{\mathrm{f}}=0.4$ [hexanes/Et $\left.{ }_{2} \mathrm{O},(1: 1)\right]$; IR $(\mathrm{NaCl}) 3453,2963,1467 \mathrm{~cm}^{-1} ;{ }^{1} \mathrm{H}$ $\operatorname{NMR}\left(\mathrm{CDCl}_{3}, 300 \mathrm{MHz}\right) \delta 1.26(\mathrm{~d}, J=6.8 \mathrm{~Hz}, 6 \mathrm{H}), 2.24$ (s, 3H), 3.17 (sept., $\left.J=6.8 \mathrm{~Hz}, 1 \mathrm{H}\right), 5.31$ (bs, 2H), $6.69(\mathrm{~d}, J=7.9 \mathrm{~Hz}, 1 \mathrm{H}), 6.73(\mathrm{~d}, J=8.1 \mathrm{~Hz}, 1 \mathrm{H}) ;{ }^{13} \mathrm{C} \mathrm{NMR}\left(\mathrm{CDCl}_{3}, 75 \mathrm{MHz}\right) \delta 141.5$, 141.0, 132.5, 121.9, 121.3, 117.4, 27.1, 22.6, 15.3; EIMS m/z (rel intensity) $166\left(\mathrm{M}^{+}, 92\right), 151$ (100), 133 (56), 105 (60). Colourless prisms were also obtained from slow evaporation of a solution of $\mathbf{9 g}$ in $\mathrm{CHCl}_{3}$. These prisms were used for X-ray analysis, and the resulting crystal structure was identical to that previously reported for this natural product (CCDC/CSD VATBOH). ${ }^{16 \mathrm{a}} \mathbf{9 g}: \mathrm{mp} 136{ }^{\circ} \mathrm{C}$ (lit. ${ }^{16 \mathrm{c}}$ $\left.138-139{ }^{\circ} \mathrm{C}\right) ; \mathrm{R}_{\mathrm{f}}=0.15$ [hexanes $/ \mathrm{Et}_{2} \mathrm{O},(1: 1)$ ]; IR (NaCl, neat) 3451, 2967, 1724, 1676, $1157 \mathrm{~cm}^{-1}$; ${ }^{1} \mathrm{H} \mathrm{NMR}\left(\mathrm{CDCl}_{3}, 300 \mathrm{MHz}\right) \delta 0.84(\mathrm{~d}, J=6.8 \mathrm{~Hz}, 3 \mathrm{H}), 0.88(\mathrm{~d}, J=6.6 \mathrm{~Hz}, 3 \mathrm{H}), 1.11(\mathrm{~d}, J=7.0 \mathrm{~Hz}$, $3 \mathrm{H}), 1.12(\mathrm{~d}, J=6.6 \mathrm{~Hz}, 3 \mathrm{H}), 1.22$ (s, 3H), 1.24 (s, 3H), 1.83 (sept., $J=6.8 \mathrm{~Hz}, 1 \mathrm{H}), 2.48$ (sept., $J=$ $6.8 \mathrm{~Hz}, 1 \mathrm{H}), 3.12(\mathrm{dd}, J=2.5,8.7 \mathrm{~Hz}, 1 \mathrm{H}), 3.13-3.17(\mathrm{~m}, 1 \mathrm{H}), 3.23(\mathrm{dd}, J=1.9,8.7 \mathrm{~Hz}, 1 \mathrm{H}), 3.35$ $(\mathrm{dq}, J=1.0,2.4,6.8 \mathrm{~Hz}, 1 \mathrm{H}), 5.84(\mathrm{~d}, J=6.8 \mathrm{~Hz}, 1 \mathrm{H}), 5.96(\mathrm{~s}, 1 \mathrm{H}) ;{ }^{13} \mathrm{C} \mathrm{NMR}\left(\mathrm{CDCl}_{3}, 100 \mathrm{MHz}\right) \delta$ $212.3,201.9,166.5,145.6,126.1,119.9,73.4,72.8,55.8,44.6,41.9,40.8,33.2,32.8,32.2,25.8$, 22.9, 20.7, 20.0, 19.2; EIMS m/z (rel intensity) $332\left(\mathrm{M}^{+}, 31\right), 315$ (16), 289 (18), 271 (40), 243 (48), 166 (42), 149 (61), 125 (100).

\section{3,10-Dihydroxy-3,10-di-isopropyl-6,12-dimethyl-tricyclo $\left[6.2 .2 .0^{2,7}\right]$ dodeca-5,11-diene-4,9-}

dione (9h). Thymol (7h) $(1.5 \mathrm{~g}, 10 \mathrm{mmol})$ was submitted to the SIBX-mediated HPD reaction according to the general procedure B. Purification by column chromatography, eluting with hexanes/Et ${ }_{2} \mathrm{O}(1: 1)$, furnished $\mathbf{1 0 \mathbf { g } ^ { 2 5 }}$ as a brown oil (229 $\left.\mathrm{mg}, 14 \%\right)$ and dimer $\mathbf{9 h}$ as a yellow solid (970 mg). This solid was crystallized from a mixture of $\mathrm{CH}_{2} \mathrm{Cl}_{2}$ and hexanes to give pure $\mathbf{9 h}$ as white needles $(830 \mathrm{mg}, 50 \%)$. Colourless prisms were also obtained from slow evaporation of a solution of $\mathbf{9 h}$ in $\mathrm{CHCl}_{3}$. These prisms were used for X-ray analysis. The resulting crystal data were deposited at the Cambridge Crystallographic Data Centre under the number CCDC 640075. 9h: mp $159.5^{\circ} \mathrm{C}$ (lit. $\left.{ }^{17} \mathrm{mp} 168-170{ }^{\circ} \mathrm{C}\right) ; \mathrm{R}_{\mathrm{f}}=0.27$ [hexanes/Et $\left.2 \mathrm{O},(1: 1)\right]$; IR ( $\mathrm{NaCl}$, neat) 3481, 2972, 2927, 1719 , 1681, 1448, $1361 \mathrm{~cm}^{-1} ;{ }^{1} \mathrm{H} \mathrm{NMR}\left(\mathrm{CDCl}_{3}, 400 \mathrm{MHz}\right) \delta 0.56(\mathrm{~d}, J=6.6 \mathrm{~Hz}, 3 \mathrm{H}), 0.81(\mathrm{~d}, J=6.8 \mathrm{~Hz}$, $3 \mathrm{H}), 0.84(\mathrm{~d}, J=7.1 \mathrm{~Hz}, 3 \mathrm{H}), 0.95(\mathrm{~d}, J=6.6 \mathrm{~Hz}, 3 \mathrm{H}), 1.50-1.65(\mathrm{~m}, 1 \mathrm{H}), 1.60$ (s, 3H), 1.76 (sept., $J$ $=6.8 \mathrm{~Hz}, 1 \mathrm{H}), 1.95(\mathrm{~s}, 3 \mathrm{H}), 2.36(\mathrm{bs}, 1 \mathrm{H}), 3.08(\mathrm{~d}, J=7.3 \mathrm{~Hz}, 1 \mathrm{H}), 3.16(\mathrm{~s}, 1 \mathrm{H}), 3.26(\mathrm{~d}, J=8.3 \mathrm{~Hz}$, $1 \mathrm{H}), 3.29(\mathrm{~d}, J=6.6 \mathrm{~Hz}, 1 \mathrm{H}), 3.79(\mathrm{~s}, 1 \mathrm{H}), 5.82(\mathrm{~d}, J=6.1 \mathrm{~Hz}, 1 \mathrm{H}), 5.97(\mathrm{~s}, 1 \mathrm{H}) ;{ }^{13} \mathrm{C} \mathrm{NMR}\left(\mathrm{CDCl}_{3}\right.$, $100 \mathrm{MHz}) \delta 214.8,201.8,155.9,135.7,126.6,125.3,78.1,77.8,57.2,47.1,41.8,37.2,37.2$, 32.5, 22.1, 21.3, 16.7, 16.6, 16.3, 16.0; ESIMS (MeOH) $m / z$ (rel intensity) $333\left(\mathrm{MH}^{+}, 100\right)$.

2-Hydroxy-2-methylnaphthalen-1(2H)-one (12). Method $A$. To a solution of 2-methylnaphthol (11a) $(100 \mathrm{mg}, 0.63 \mathrm{mmol})$ in THF $(1.5 \mathrm{~mL})$ was added SIBX $(0.394 \mathrm{~g}, 0.69 \mathrm{mmol})$ as a solid in one portion. The resulting suspension was stirred at room temperature for $14 \mathrm{~h}$, after which time the 
reaction mixture was poured into ice-cold water $(5 \mathrm{~mL})$, neutralized with aqueous $1 \mathrm{M} \mathrm{NaOH}$, and extracted with $\mathrm{CH}_{2} \mathrm{Cl}_{2}(2 \times 5 \mathrm{~mL})$. The combined organic phases were then washed with water (10 $\mathrm{mL}$ ), dried over $\mathrm{Na}_{2} \mathrm{SO}_{4}$, filtered and evaporated to furnish orthoquinol $\mathbf{1 2}^{26}(110 \mathrm{mg}, 100 \%)$ as a red oil. Method B. Treatment of the trimethylsilylated 2-methylnaphthol 11b (50 mg, $0.2 \mathrm{mmol})$ in DMSO (1 mL) with SIBX (255 mg, $0.5 \mathrm{mmol}$, i.e., 2.5 equiv of IBX) was run at room temperature for $2 \mathrm{~h}$, after which time the reaction mixture was diluted with EtOAc $(20 \mathrm{~mL})$, washed with saturated aqueous $\mathrm{NaHCO}_{3}(3 \times 5 \mathrm{~mL})$, and brine $(5 \mathrm{~mL})$, dried over $\mathrm{Na}_{2} \mathrm{SO}_{4}$, filtered and evaporated to furnish orthoquinol $12(35 \mathrm{mg}, 100 \%)$ as a red oil. IR ( NaCl, neat) 3460, 2881, 1686, 1599, 1291 $\mathrm{cm}^{-1} ;{ }^{1} \mathrm{H} \mathrm{NMR}\left(\mathrm{CDCl}_{3}, 250 \mathrm{MHz}\right) \delta 1.43(\mathrm{~s}, 3 \mathrm{H}), 6.30(\mathrm{~d}, J=9.8 \mathrm{~Hz}, 1 \mathrm{H}), 6.44(\mathrm{~d}, J=9.8 \mathrm{~Hz}, 1 \mathrm{H})$, $7.17(\mathrm{~d}, J=7.6 \mathrm{~Hz}, 1 \mathrm{H}), 7.31(\mathrm{dt}, J=1.2,7.6 \mathrm{~Hz}, 1 \mathrm{H}), 7.55(\mathrm{dt}, J=1.2,7.6 \mathrm{~Hz}, 1 \mathrm{H}), 7.95(\mathrm{~d}, J=7.9$ $\mathrm{Hz}, 1 \mathrm{H}) ;{ }^{13} \mathrm{C} \mathrm{NMR}\left(\mathrm{CDCl}_{3}, 62.9 \mathrm{MHz}\right) \delta 204.1,137.6,136.9,135.0,128.0,127.9,127.3,127.0$, 124.6, 75.2, 28.2; EIMS m/z (rel. intensity) $174\left(\mathrm{M}^{+}, 1\right) 131$ (7), 43 (29), 28 (100).

1,2-Naphthoquinone (see Scheme 1). To a solution of 1-naphthol (100 mg, $0.70 \mathrm{mmol})$ in THF $(15 \mathrm{~mL})$ was added SIBX (435 $\mathrm{mg}, 0.76 \mathrm{mmol}$ ) as a solid in one portion. The resulting suspension was stirred was stirred at room temperature in the dark for $14 \mathrm{~h}$, after which time water $(30 \mathrm{~mL})$ was added. This mixture was then extracted with $\mathrm{CH}_{2} \mathrm{Cl}_{2}(2 \times 15 \mathrm{~mL})$, and the combined organic phases were washed with saturated aqueous $\mathrm{NaHCO}_{3}(6 \times 15 \mathrm{~mL})$ and water $(20 \mathrm{~mL})$, dried over $\mathrm{Na}_{2} \mathrm{SO}_{4}$, filtrated and evaporated to give 1,2-naphthoquinone (108 $\mathrm{mg}, 99 \%)$ as a red solid. ${ }^{8 \mathrm{a}, 27}$

Scheme 1. Plausible Mechanism of the SIBX-Mediated Oxidation of 1-Naphthol into 1,2Naphthoquinone
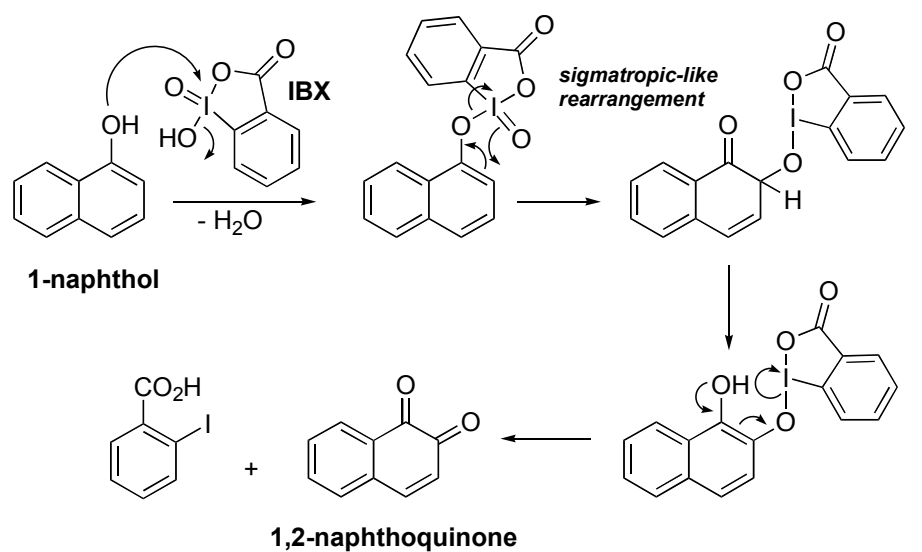
Scheme 2. Preparation of Functionalized Naphthols 11d-e

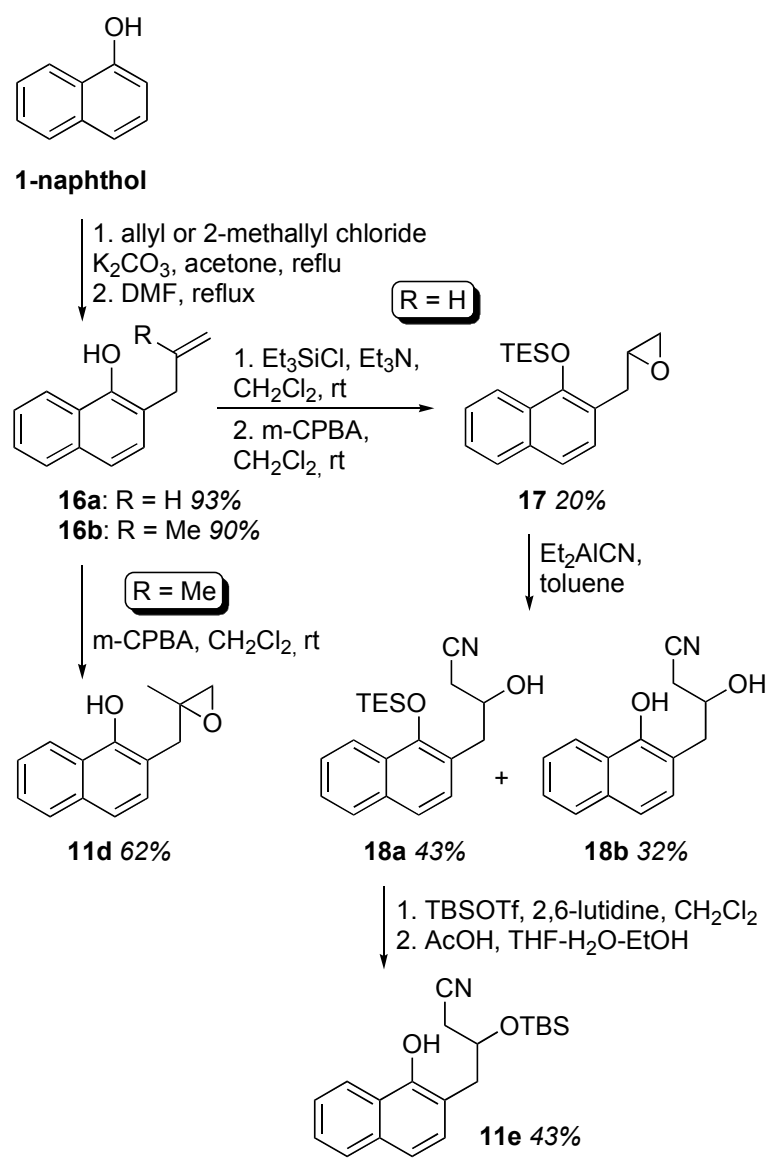

The epoxide 11d was generated in three steps from 1-naphthol (Scheme 2). After $O$-allylation of 1-naphthol with 2-methylallyl chloride and Claisen rearrangement in refluxing $\mathrm{DMF},{ }^{28}$ the resulting 2-(2-methylallyl)-naphthalen-1-ol (16a) was treated with $m$-CPBA in $\mathrm{CH}_{2} \mathrm{Cl}_{2}$ to furnish 11d in $62 \%$ yield. Similarly, the $O$-allylation of 1-naphthol with allyl chloride, followed by a Claisen rearrangement, gave 2-allylnaphthalen-1-ol (16b) in 90\% yield (Scheme 2). ${ }^{28}$ This allylated naphthol was first protected as a triethylsilyloxy derivative and then converted to the epoxide $\mathbf{1 7}$ by treatment with $m$-CPBA in $\mathrm{CH}_{2} \mathrm{Cl}_{2}$. Opening of this epoxide with the Nagata's reagent in toluene gave a mixture of triethylsilylated naphtholic and free naphtholic $\beta$-hydroxynitriles $\mathbf{1 8 a}$ and $\mathbf{1 8 b}$ in $43 \%$ and $32 \%$, respectively. Hence, the tertiary alcohol of the $\beta$-hydoxynitrile 18a was protected as a tertbutyldimethylsilyl ether, and the triethylsilyloxy group was selectively cleaved using acetic acid to furnish the naphthol 11e in $43 \%$ over the two steps (Scheme 2).

2-[(2-methyloxiran-2-yl)methyl]naphthalen-1-ol (11d). To a solution of 2-(2-methylallyl)naphthol $(\mathbf{1 6 b})^{28}(200 \mathrm{mg}, 1.0 \mathrm{mmol})$ in $\mathrm{CH}_{2} \mathrm{Cl}_{2}(10 \mathrm{~mL})$ at $0{ }^{\circ} \mathrm{C}$ was slowly added $m$-CPBA (373 $\mathrm{mg}, 1.5 \mathrm{mmol}$ ). This reaction mixture was stirred at $0{ }^{\circ} \mathrm{C}$ for $6 \mathrm{~h}$, after which time it was carefully 
quenched with saturated aqueous $\mathrm{Na}_{2} \mathrm{SO}_{3}(10 \mathrm{~mL})$ and extracted with $\mathrm{CH}_{2} \mathrm{Cl}_{2}(3 \times 20 \mathrm{~mL})$. The combined organic phases were washed with saturated aqueous $\mathrm{NaHCO}_{3}(4 \times 30 \mathrm{~mL})$, brine $(20 \mathrm{~mL})$, dried over $\mathrm{Na}_{2} \mathrm{SO}_{4}$, and evaporated to give a residue, which was purified by column chromatography, eluting with cyclohexane/ $\mathrm{Et}_{2} \mathrm{O}(3: 1)$, to afford 11d (133 mg, 62\%) as a pale yellow oil: IR ( $\mathrm{NaCl}$, neat) 3308, $1297 \mathrm{~cm}^{-1} ;{ }^{1} \mathrm{H} \mathrm{NMR}\left(\mathrm{CDCl}_{3}, 400 \mathrm{MHz}\right) \delta 1.38(\mathrm{~s}, 3 \mathrm{H}), 2.87(\mathrm{~d}, J=3.6 \mathrm{~Hz}, 1 \mathrm{H}), 2.88(\mathrm{~d}, J$ $=15.6 \mathrm{~Hz}, 1 \mathrm{H}), 2.96(\mathrm{~d}, J=3.9 \mathrm{~Hz}, 1 \mathrm{H}), 3.28(\mathrm{~d}, J=14.9 \mathrm{~Hz}, 1 \mathrm{H}), 7.18(\mathrm{~d}, J=8.3 \mathrm{~Hz}, 1 \mathrm{H}), 7.40(\mathrm{~d}, J$ $=8.1 \mathrm{~Hz}, 1 \mathrm{H}), 7.49-7.52(\mathrm{~m}, 2 \mathrm{H}), 7.80(\mathrm{~d}, J=7.2 \mathrm{~Hz}, 1 \mathrm{H}), 8.28(\mathrm{~s}, 1 \mathrm{H}), 8.39(\mathrm{~d}, J=9.0 \mathrm{~Hz}, 1 \mathrm{H}) ;{ }^{13} \mathrm{C}$ $\mathrm{NMR}\left(\mathrm{CDCl}_{3}, 100 \mathrm{MHz}\right) \delta 151.4,134.1,129.4,127.1,125.9,125.6,125.0,122.4,119.5,116.0,60.0$, 55.6, 39.5, 20.0; EIMS $m / z$ (rel. intensity) 214 (88), 156 (100); Anal. calcd for $\mathrm{C}_{14} \mathrm{H}_{14} \mathrm{O}_{2}$ : C, 78.48; $\mathrm{H}$, 6.59. Found: C, 78.20; H, 6.50.

2-(Oxiran-2-yl)methyl-1-triethylsilyloxynaphthalene (17). Triethylamine $(6.3 \mathrm{~mL}$ g, 45.3 mmol) was added dropwise to a stirred ice-cold solution of 2-allylnaphthol (16a) ${ }^{28}(6.4 \mathrm{~g}, 34.8$ mmol) in $\mathrm{CH}_{2} \mathrm{Cl}_{2}(200 \mathrm{~mL})$. This mixture was then stirred for $5 \mathrm{~min}$, after which time $\mathrm{Et}_{3} \mathrm{SiCl}(6.4$ $\mathrm{mL}, 38.3 \mathrm{mmol}$ ) was slowly added at $0{ }^{\circ} \mathrm{C}$. After stirring for $12 \mathrm{~h}$ at this temperature, the reaction mixture was diluted with $\mathrm{CH}_{2} \mathrm{Cl}_{2}(80 \mathrm{~mL})$, washed with aqueous $1 \mathrm{M} \mathrm{H}_{3} \mathrm{PO}_{4}(2 \times 20 \mathrm{~mL})$, brine $(2 \times$ $20 \mathrm{~mL}$ ), dried over $\mathrm{Na}_{2} \mathrm{SO}_{4}$, and evaporated to give 2-allyl-1-triethylsilyloxynaphthalene (10.2 g, 98\%) as a reddish oil: IR ( $\mathrm{NaCl}$, neat) 2956, 2917, $2877 \mathrm{~cm}^{-1} ;{ }^{1} \mathrm{H} \mathrm{NMR}\left(\mathrm{CDCl}_{3}, 400 \mathrm{MHz}\right) \delta 0.92-$ 0.97 (q, $J=7.8 \mathrm{~Hz}, 6 \mathrm{H}), 1.05-1.11(\mathrm{q}, J=8.1 \mathrm{~Hz}, 9 \mathrm{H}), 3.67$ (d, $J=6.4 \mathrm{~Hz}, 2 \mathrm{H}), 5.21-5.25$ (m, 2H), 6.07-6.21 (m, 1H), $7.38(\mathrm{~d}, J=8.6 \mathrm{~Hz}, 1 \mathrm{H}), 7.48-7.57(\mathrm{~m}, 3 \mathrm{H}), 7.85(\mathrm{~d}, J=8.1 \mathrm{~Hz}, 1 \mathrm{H}), 8.18(\mathrm{~d}, J=$ $8.1 \mathrm{~Hz}, 1 \mathrm{H}) ;{ }^{13} \mathrm{C} \mathrm{NMR}\left(\mathrm{CDCl}_{3}, 100 \mathrm{MHz}\right) \delta 149.3,137.4,134.2,128.7,128.6,128.1,125.7,125.5$, 124.9, 122.9, 121.9, 116.3, 34.9, 7.3, 6.2; EIMS m/z (rel. intensity) 298 ( $\left.\mathrm{M}^{+}, 100\right), 217$ (93); HMRS (EI) calcd for $\mathrm{C}_{19} \mathrm{H}_{26} \mathrm{OSi} 298.1753$, found 298.1741.

This silylated allylnaphthol $(5.0 \mathrm{~g}, 16.8 \mathrm{mmol})$ was dissolved into $\mathrm{CH}_{2} \mathrm{Cl}_{2}(150 \mathrm{~mL})$. To the resulting ice-cooled solution was added slowly $m$-CPBA $(6.2 \mathrm{~g}, 25.1 \mathrm{mmol})$. This reaction mixture was stirred at $0{ }^{\circ} \mathrm{C}$ for 3 days, after which time it was carefully quenched with a saturated aqueous $\mathrm{Na}_{2} \mathrm{SO}_{3}(20 \mathrm{~mL})$ and separated. The aqueous phase was extracted with $\mathrm{CH}_{2} \mathrm{Cl}_{2}(3 \times 50 \mathrm{~mL})$, and the combined organic phases were washed with saturated aqueous $\mathrm{NaHCO}_{3}(4 \times 40 \mathrm{~mL})$ and brine $(30$ $\mathrm{mL}$ ), dried over $\mathrm{Na}_{2} \mathrm{SO}_{4}$, and evaporated to give a residue, which was purified by column chromatography, eluting with cyclohexane/ $\mathrm{Et}_{2} \mathrm{O}(10: 1)$, to furnish $17(1.1 \mathrm{~g}, 20 \%)$ as a yellow oil: IR (NaCl, neat) 2957, 2913, $2877 \mathrm{~cm}^{-1} ;{ }^{1} \mathrm{H} \mathrm{NMR}\left(\mathrm{CDCl}_{3}, 400 \mathrm{MHz}\right) \delta 0.82-0.86(\mathrm{~m}, 6 \mathrm{H}), 0.96-1.00$ (m, 9H), $2.61(\mathrm{dd}, J=4.9,2.5 \mathrm{~Hz}, 1 \mathrm{H}), 2.80(\mathrm{dd}, J=4.6,4.2 \mathrm{~Hz}, 1 \mathrm{H}), 2.95(\mathrm{dd}, J=14.4,5.4 \mathrm{~Hz}$, $1 \mathrm{H}), 3.16(\mathrm{dd}, J=14.4,5.4 \mathrm{~Hz}, 1 \mathrm{H}), 3.24(\mathrm{~m}, 1 \mathrm{H}), 7.37-7.49(\mathrm{~m}, 4 \mathrm{H}), 7.79(\mathrm{~d}, J=7.4 \mathrm{~Hz}, 1 \mathrm{H}), 8.07$ $(\mathrm{d}, J=7.6 \mathrm{~Hz}, 1 \mathrm{H}) ;{ }^{13} \mathrm{C} \mathrm{NMR}\left(\mathrm{CDCl}_{3}, 100 \mathrm{MHz}\right) \delta 149.4,133.9,128.2,128.1,127.7,125.5,125.2$, 
122.5, 122.1, 121.6, 51.9, 47.0, 33.4, 6.8, 5.7; EIMS $m / z$ (rel. intensity) $314\left(\mathrm{M}^{+}, 85\right), 285(36), 257$ (89), 227 (92); HMRS (EI) calcd for $\mathrm{C}_{19} \mathrm{H}_{26} \mathrm{O}_{2} \mathrm{Si} 314.1702$, found 314.1711.

\section{2-[(3-cyano-2-hydroxy)propyl]-1-triethylsilyloxynaphthalene (18a) and 2-[(3-cyano-2-} hydroxy)propyl]naphthalen-1-ol (18b). $\mathrm{Et}_{2} \mathrm{AlCN}(1.0 \mathrm{M}$ in toluene, $8.6 \mathrm{ml}, 8.6 \mathrm{mmol})$ was added dropwise to a solution of $17(675 \mathrm{mg}, 2.1 \mathrm{mmol})$ in toluene $(20 \mathrm{~mL})$ at $-40{ }^{\circ} \mathrm{C}$. This reaction mixture was stirred at this temperature for $40 \mathrm{~min}$, after which time it was quenched with aqueous $1 \mathrm{M} \mathrm{HCl}$ solution $(1 \mathrm{~mL})$. The resulting mixture was stirred at $\mathrm{rt}$ for $40 \mathrm{~min}$ and separated. The aqueous phase was extracted with EtOAc $(2 \times 15 \mathrm{~mL})$, and the combined organic phases were washed with aqueous $1 \mathrm{M} \mathrm{HCl}(2 \times 10 \mathrm{~mL})$ and brine $(20 \mathrm{~mL})$, dried over $\mathrm{Na}_{2} \mathrm{SO}_{4}$, and evaporated to give a residue, which was purified by column chromatography, eluting with cyclohexane/ $\mathrm{Et}_{2} \mathrm{O}(2: 1)$, to furnish 18a (311 $\mathrm{mg}, 43 \%$ ) and $\mathbf{1 8 b}(156 \mathrm{mg}, 32 \%)$ as pale yellow oils. 18a: IR ( NaCl, neat) 2926, $2252 \mathrm{~m}^{-1} ;{ }^{1} \mathrm{H}$ NMR $\left(\mathrm{CDCl}_{3}, 400 \mathrm{MHz}\right) \delta 0.89-0.96(\mathrm{~m}, 6 \mathrm{H}), 1.03-1.07(\mathrm{~m}, 9 \mathrm{H}), 2.52-2.63(\mathrm{~m}, 2 \mathrm{H}), 2.83(\mathrm{~s}, 1 \mathrm{H})$, 3.13-3.25 (m, 2H), 4.30-4.36 (m, 1H), 7.35 (d, 1H), 7.52-7.58 (m, 3H), 7.86-7.89 (m, 1H), 8.10-8.13 $(\mathrm{m}, 1 \mathrm{H}) ;{ }^{13} \mathrm{C} \mathrm{NMR}\left(\mathrm{CDCl}_{3}, 100 \mathrm{MHz}\right) \delta 150.0,134.2,128.6,128.0,127.8,126.0,125.5,122.4$, 122.3, 121.5, 117.6, 68.3, 38.2, 25.2, 6.7, 5.7; EIMS m/z (rel. intensity) $341\left(\mathrm{M}^{+}, 77\right), 312$ (66), 271 (96); HMRS (EI) calcd for $\mathrm{C}_{20} \mathrm{H}_{27} \mathrm{NO}_{2} \mathrm{Si} 341.1811$, found 341.1800. 18b: IR (NaCl, neat) 3372, 2952, $2257 \mathrm{~cm}^{-1}$; ${ }^{1} \mathrm{H}$ NMR $\left(\mathrm{CDCl}_{3}, 400 \mathrm{MHz}\right) \delta 2.49(\mathrm{~d}, \mathrm{~J}=6.1 \mathrm{~Hz}, 2 \mathrm{H}), 2.96-3.08(\mathrm{~m}, 2 \mathrm{H}), 4.13$ (s, $1 \mathrm{H}), 4.32-4.37(\mathrm{~m}, 1 \mathrm{H}), 7.10(\mathrm{~d}, \mathrm{~J}=8.3 \mathrm{~Hz}, 1 \mathrm{H}), 7.37(\mathrm{~d}, \mathrm{~J}=8.1 \mathrm{~Hz}, 1 \mathrm{H}), 7.45-7.47(\mathrm{~m}, 2 \mathrm{H}), 7.75-$ $7.78(\mathrm{~m}, 1 \mathrm{H}), 8.23-8.25(\mathrm{~m}, 1 \mathrm{H}) ;{ }^{13} \mathrm{C} \mathrm{NMR}\left(\mathrm{CDCl}_{3}, 100 \mathrm{MHz}\right) \delta 151.0,134.1,129.2,127.4,126.2$, 125.6, 125.4, 122.1, 120.1, 117.6, 116.2, 69.5, 38.2, 25.2; EIMS m/z (rel. intensity) $227\left(\mathrm{M}^{+}, 100\right)$, 210 (87) HMRS (EI) calcd for $\mathrm{C}_{13} \mathrm{H}_{13} \mathrm{NO}_{2} 227.0946$, found 227.0955.

2-[(2-tert-butyldimethylsilyloxy-3-cyano)propyl]naphthalen-1-ol (11e). TBDMSOTf (143 $\mu \mathrm{L}$, $0.62 \mathrm{mmol}$ ) was added to a mixture of $\mathbf{1 8 a}(74 \mathrm{mg}, 0.2 \mathrm{mmol})$ and 2,6-lutidine ( $73 \mu \mathrm{L}, 0.62 \mathrm{mmol})$ in $\mathrm{CH}_{2} \mathrm{Cl}_{2}(4 \mathrm{~mL})$ at $0{ }^{\circ} \mathrm{C}$. After stirring for $3 \mathrm{~h}$ at this temperature, saturated aqueous $\mathrm{NaHCO}_{3}(5 \mathrm{~mL})$ was added to the reaction mixture, which was then separated. The aqueous phase was extracted with $\mathrm{Et}_{2} \mathrm{O}(20 \mathrm{~mL})$, and the combined organic phases were washed with brine $(20 \mathrm{~mL})$, dried over $\mathrm{Na}_{2} \mathrm{SO}_{4}$, and evaporated to furnish the desired silylated derivative (98 mg), which was used in the next step without purification: IR ( $\mathrm{NaCl}$, neat) 2956, 2930, 2880, 2957, $2349 \mathrm{~cm}^{-1}$; ${ }^{1} \mathrm{H} \mathrm{NMR}\left(\mathrm{CDCl}_{3}\right.$, $400 \mathrm{MHz}) \delta-0.14(\mathrm{~s}, 3 \mathrm{H}), 0.08(\mathrm{~s}, 3 \mathrm{H}), 0.82-0.89(\mathrm{~m}, 18 \mathrm{H}), 0.93-0.99(\mathrm{~m}, 6 \mathrm{H}), 2.35-2.48(\mathrm{~m}, 2 \mathrm{H})$, 3.00-3.11 (m, 2H), 4.21-4.26 (m, 1H), 7.26 (d, J= 8.3 Hz, 1H), 7.44-7.47 (m, 3H), $7.78(\mathrm{~d}, J=7.1 \mathrm{~Hz}$, $1 \mathrm{H}), 8.04(\mathrm{~d}, J=8.6 \mathrm{~Hz}, 1 \mathrm{H}) ;{ }^{13} \mathrm{C} \mathrm{NMR}\left(\mathrm{CDCl}_{3}, 100 \mathrm{MHz}\right) \delta 149.8,134.0,129.0,128.1,127.7$, 125.7, 125.2, 122.5, 122.0, 121.6, 118.0, 68.9, 38.7, 25.7, 25.6, 17.9, 6.7, 5.7, -3.0, -4.9; HMRS (EI) calcd for $\mathrm{C}_{26} \mathrm{H}_{41} \mathrm{NO}_{2} \mathrm{Si}_{2}$ 455.2676, found 455.2654. 
This disilylated compound (200 $\mathrm{mg}$ from two runs, $0.44 \mathrm{mmol}$ ) was then dissolved in $\mathrm{THF} / \mathrm{AcOH} / \mathrm{H}_{2} \mathrm{O} / \mathrm{EtOH}(1: 6: 3: 2,22 \mathrm{~mL})$. The resulting mixture was stirred at $\mathrm{rt}$ for 4 days, after which time it was extracted with $\mathrm{Et}_{2} \mathrm{O}(20 \mathrm{~mL})$, washed with saturated aqueous $\mathrm{NaHCO}_{3}(20 \mathrm{~mL})$, dried over $\mathrm{Na}_{2} \mathrm{SO}_{4}$ and evaporated to give a residue, which was purified by column chromatography, eluting with cyclohexane/Et ${ }_{2} \mathrm{O}(3: 1)$, to furnish $11 \mathrm{e}(64 \mathrm{mg}, 43 \%)$ as a pale yellow oil: IR $(\mathrm{NaCl}$, neat) 3289, 2955, 2929, 2857, $2252 \mathrm{~cm}^{-1} ;{ }^{1} \mathrm{H} \mathrm{NMR}\left(\mathrm{CDCl}_{3}, 400 \mathrm{MHz}\right) \delta 0.07$ (s, 3H), $0.18(\mathrm{~s}, 3 \mathrm{H})$, 0.98 (s, 9H), 1.44 (s, 1H), 2.46 (s, 2H), 3.13-3.24 (m, 2H), 4.45 (s, 1H), 7.18 (d, J= 8.0 Hz, 1H), 7.37$7.40(\mathrm{~m}, 1 \mathrm{H}), 7.47(\mathrm{~d}, 4.8 \mathrm{~Hz}, 1 \mathrm{H}), 7.77-7.78(\mathrm{~m}, 1 \mathrm{H}), 7.94(\mathrm{~d}, J=2.7 \mathrm{~Hz}, 1 \mathrm{H}), 8.27(\mathrm{~d}, J=4.8 \mathrm{~Hz}$, $1 \mathrm{H}) ;{ }^{13} \mathrm{C} \mathrm{NMR}\left(\mathrm{CDCl}_{3}, 100 \mathrm{MHz}\right) \delta 151.1,134.2,129.4,127.3,126.1,125.4,125.2,122.2,120.0$, 116.9, 115.2, 70.4, 39.1, 25.7, 24.7, 18.0, -4.9; HMRS (EI) calcd for $\mathrm{C}_{20} \mathrm{H}_{27} \mathrm{NO}_{2} \mathrm{Si} 341.1811$, found 341.1812 .

\section{Additional references}

(25) Schwarz, K.; Ernst, H.; Ternes, W. J. Sci. Food Agric. 1996, 70, 217-223.

(26) Krohn, K.; Brüggmann, K.; Doering, D.; Jones, P. G. Chem. Ber. 1992, 125, 2439-2442.

(27) Krohn, K.; Rieger, H.; Brüggmann, K. Synthesis 1990, 1141-1143.

(28) Kongkathip, N.; Kongkathip, B.; Siripong, P.; Sangma, C.; Luangkamin, S.; Niyomdecha, M.; Pattanapa, S.; Piyaviriyagul, S.; Kongsaeree, P. Bioorg. Med. Chem. 2003, 11, 3179-3191. 


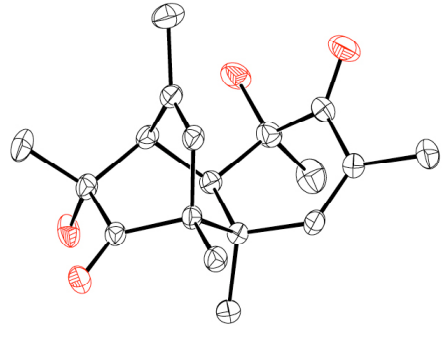

CCDC 640072

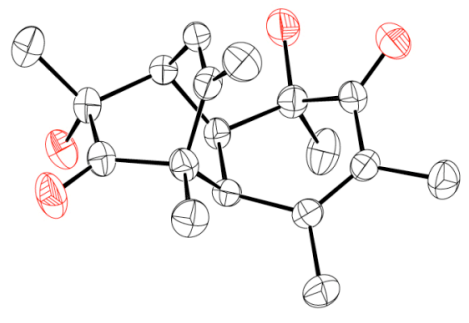

CCDC 640073

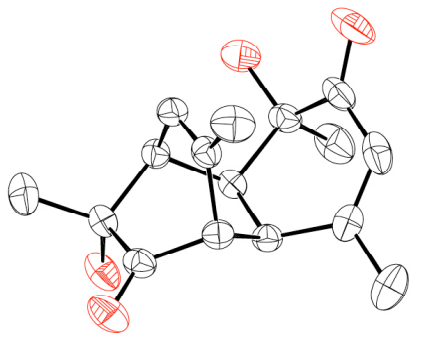

CCDC 640074

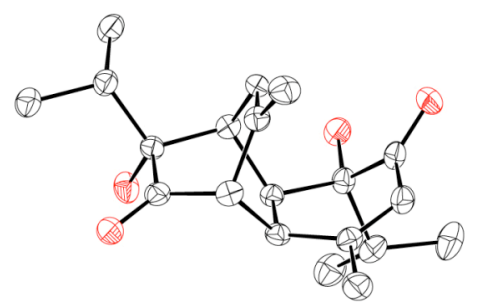

CCDC 640075
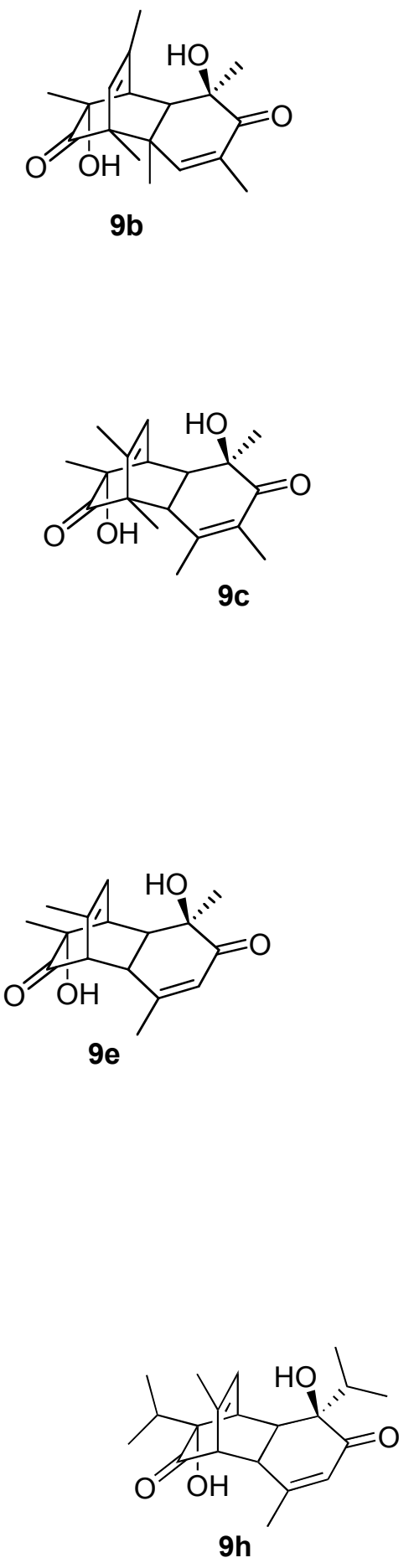
Table 3. Influence of an acidic medium on the IBX-mediated HPD of 2,6-dimethylphenol (7a)

\begin{tabular}{|c|c|c|c|c|}
\hline entry $^{a}$ & acid & reaction time & $\begin{array}{l}\text { IBA-bearing } \\
\text { dimer }\end{array}$ & free dimer $(\mathbf{9 a})$ \\
\hline 1 & SIBX & $12 \mathrm{~h}$ & & $\checkmark$ \\
\hline 2 & IBX & 2 days & $\checkmark$ & \\
\hline 3 & $\begin{array}{c}\text { IBX }+ \text { benzoic acid ( } 3 \\
\text { equiv) }\end{array}$ & 2 days & $\checkmark$ & \\
\hline 4 & $\begin{array}{c}\text { IBX }+ \text { isophthalic acid ( } 3 \\
\text { equiv) }\end{array}$ & 2 days & $\checkmark$ & \\
\hline 5 & $\begin{array}{l}\text { IBX + benzoic }(22 \%) \text { and } \\
\text { isophthalic }(29 \%) \text { acids } \\
\text { (i.e., SIBX formulation) }\end{array}$ & 2 days & $\checkmark$ & \\
\hline 6 & IBX + TFA ( 3 equiv) & $1 \mathrm{~h}$ & & $\checkmark$ \\
\hline 7 & IBX, then TFA ( 1 equiv) ${ }^{b}$ & 2 days & & $\checkmark$ \\
\hline 8 & IBX + TFA (0.1 equiv) & $12 \mathrm{~h}$ & & $\checkmark$ \\
\hline 9 & $\mathrm{IBX}+\mathrm{CSA}(0.1$ equiv $)$ & $12 \mathrm{~h}$ & & $\checkmark$ \\
\hline 10 & $\begin{array}{c}\mathrm{IBX}+\mathrm{HCl} \text { conc. }(0.1 \\
\text { equiv })\end{array}$ & $12 \mathrm{~h}$ & & $\checkmark$ \\
\hline
\end{tabular}

${ }^{a}$ All reactions were run on 2,6-dimethylphenol (7a) $(100 \mathrm{mg}, 0.82 \mathrm{mmol})$ in THF $(2 \mathrm{~mL})$ at $\mathrm{rt}$ using IBX (1.0 equiv), except the reference reaction (entry 1) for which SIBX (1.1 equiv) was used. ${ }^{b}$ TFA was added after running the reaction for 2 days; the reaction mixture was then stirred at $\mathrm{rt} 1 \mathrm{~h}$. Below are examples of ${ }^{1} \mathrm{H}$ NMR spectra of crude reaction mixtures before (top, IBA-bearing dimer) and after (bottom, free dimer) treatment with TFA:
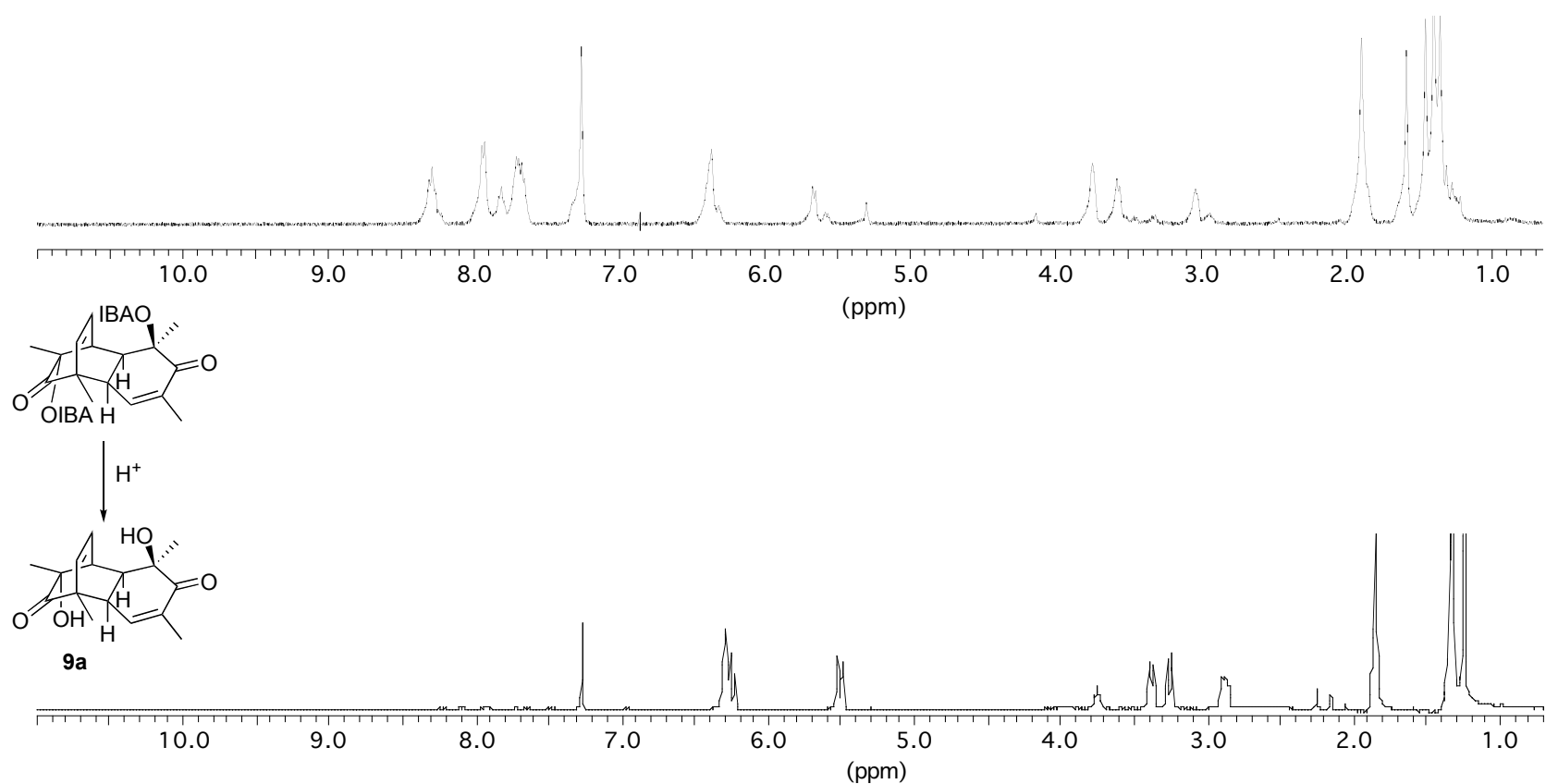
JGD48-1 - DPX400 - 091005 -



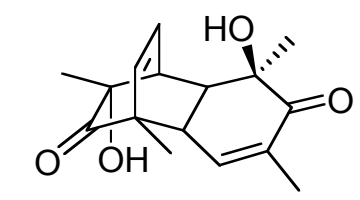

$9 a$

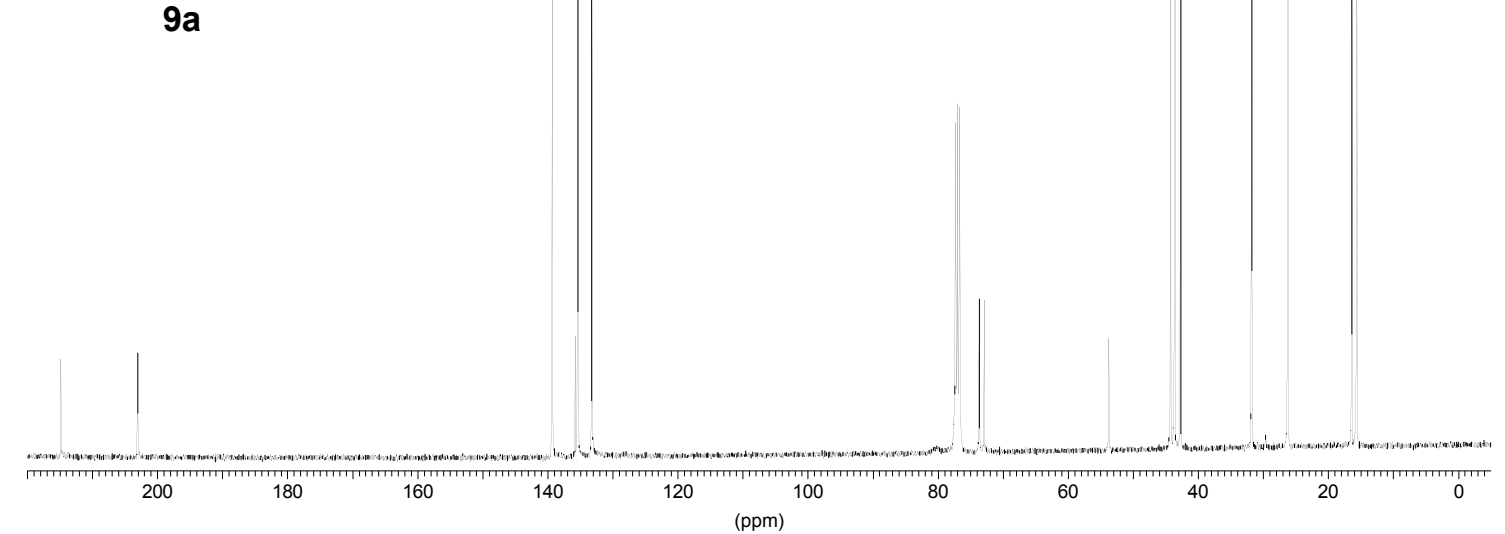

JGD48-1 - 300MHz - 260406 -

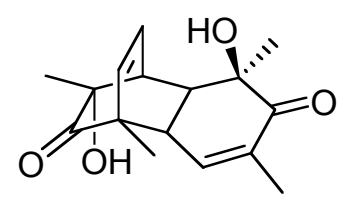

9a
ॠై

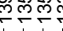

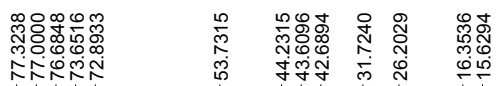

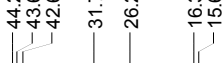

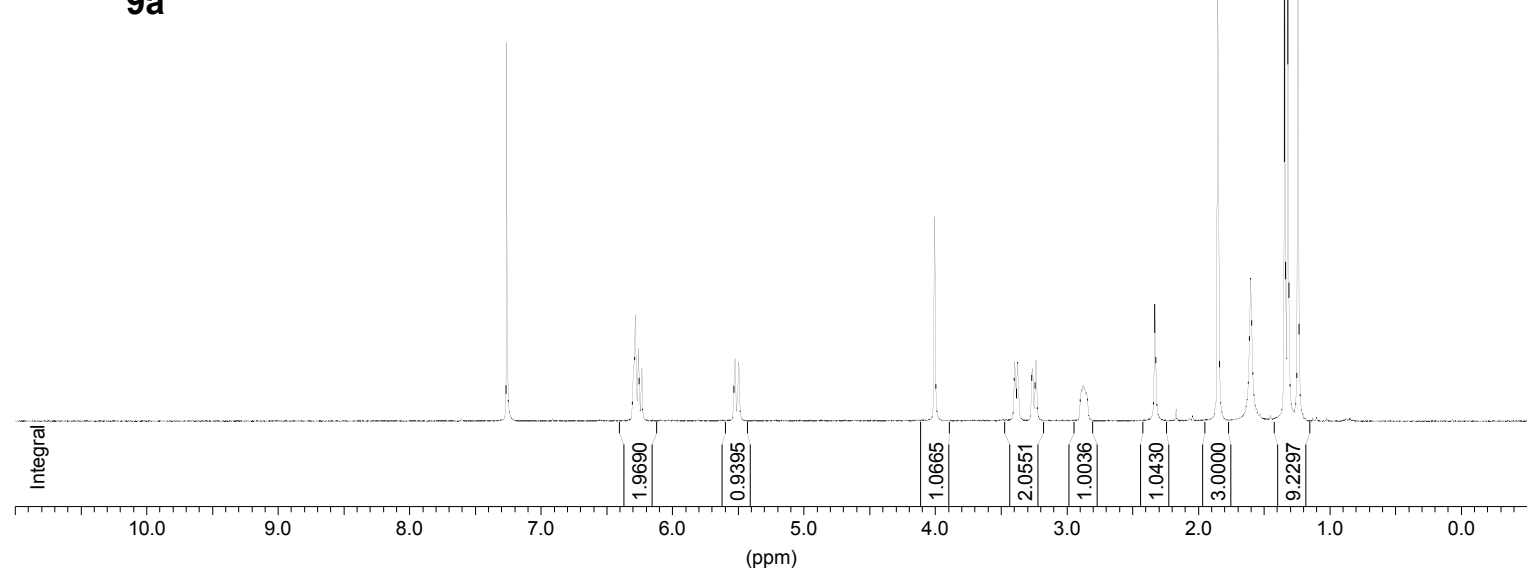




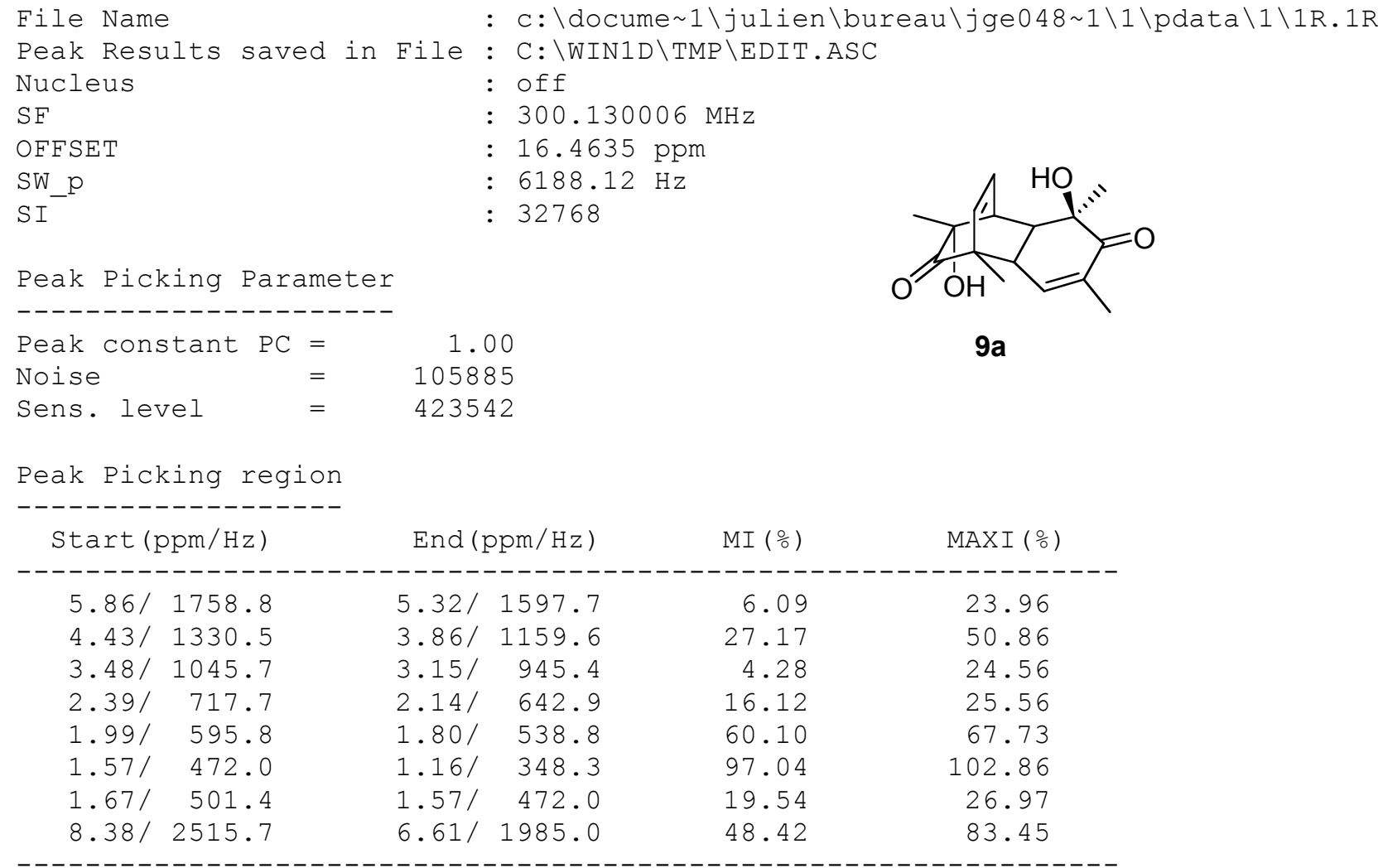

Peak Picking results

\begin{tabular}{|c|c|c|c|c|c|}
\hline Peak & Nr. Data Point & Frequency & $\mathrm{PPM}$ & Intensity & ㅇt. \\
\hline 1 & 14627 & 2178.94 & 7.2600 & 167867168 & 54.3 \\
\hline 2 & 17387 & 1657.73 & 5.5234 & 27754644 & 9.0 \\
\hline 3 & 17430 & 1649.61 & 5.4963 & 26035728 & 8.4 \\
\hline 4 & 19800 & 1202.04 & 4.0051 & 90750840 & 29.3 \\
\hline 5 & 20770 & 1018.86 & 3.3947 & 26100242 & 8.4 \\
\hline 6 & 20806 & 1012.06 & 3.3721 & 26257764 & 8.5 \\
\hline 7 & 20978 & 979.58 & 3.2639 & 23403428 & 7.6 \\
\hline 8 & 20987 & 977.88 & 3.2582 & 23146184 & 7.5 \\
\hline 9 & 21022 & 971.27 & 3.2362 & 27259416 & 8.8 \\
\hline 10 & 21031 & 969.57 & 3.2305 & 26779684 & 8.7 \\
\hline 11 & 22463 & 699.14 & 2.3295 & 51884976 & 16.8 \\
\hline 12 & 23224 & 555.43 & 1.8506 & 187980608 & 60.8 \\
\hline 13 & 23622 & 480.27 & 1.6002 & 63490100 & 20.5 \\
\hline 14 & 24031 & 403.03 & 1.3429 & 305954624 & 98.9 \\
\hline 15 & 24072 & 395.29 & 1.3171 & 309223520 & 100.0 \\
\hline 16 & 24196 & 371.87 & 1.2390 & 300153600 & 97.1 \\
\hline
\end{tabular}


JGD47-1 - DPX400 - 251005 -

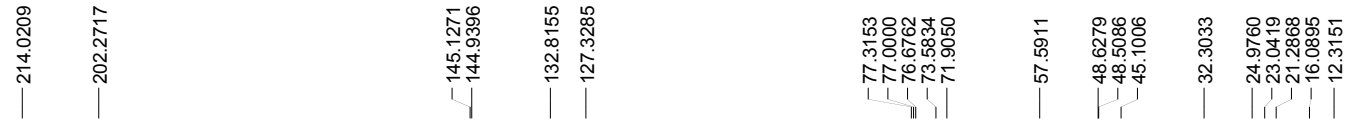

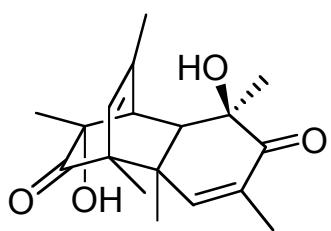

$9 b$

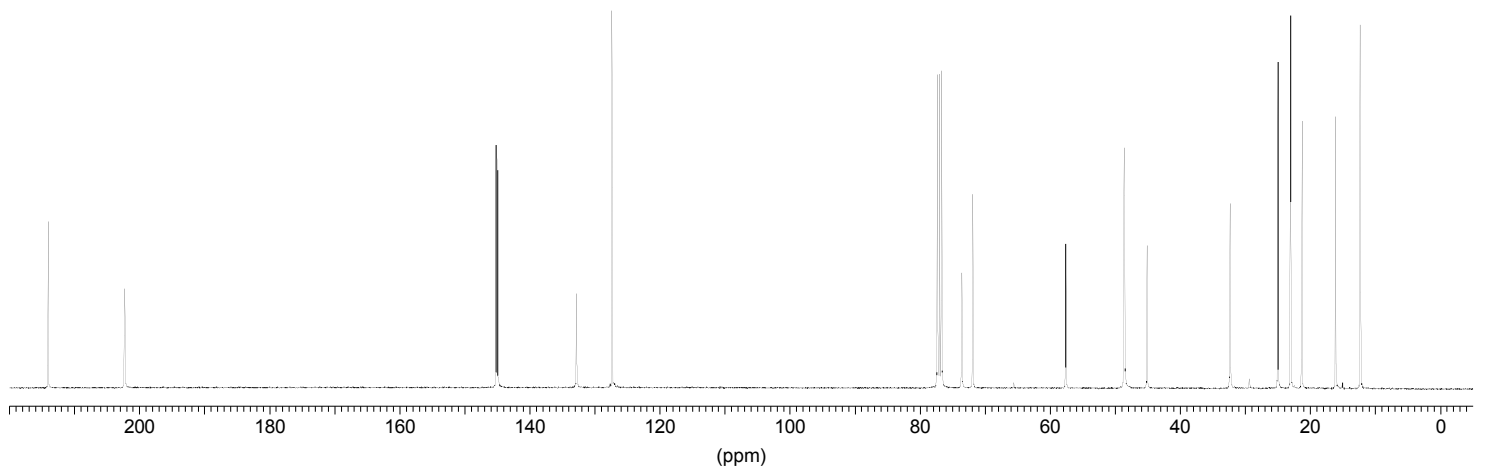

JGD47-1 - 300MHz - 260406 -

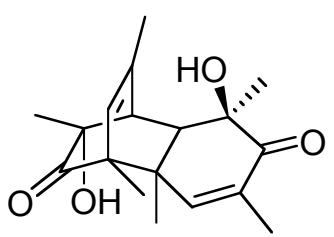

$9 b$

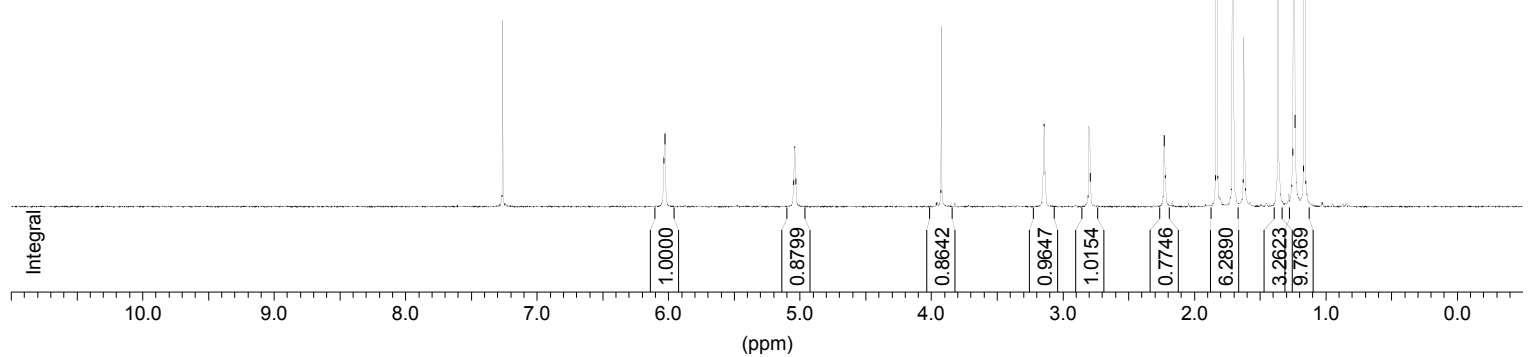




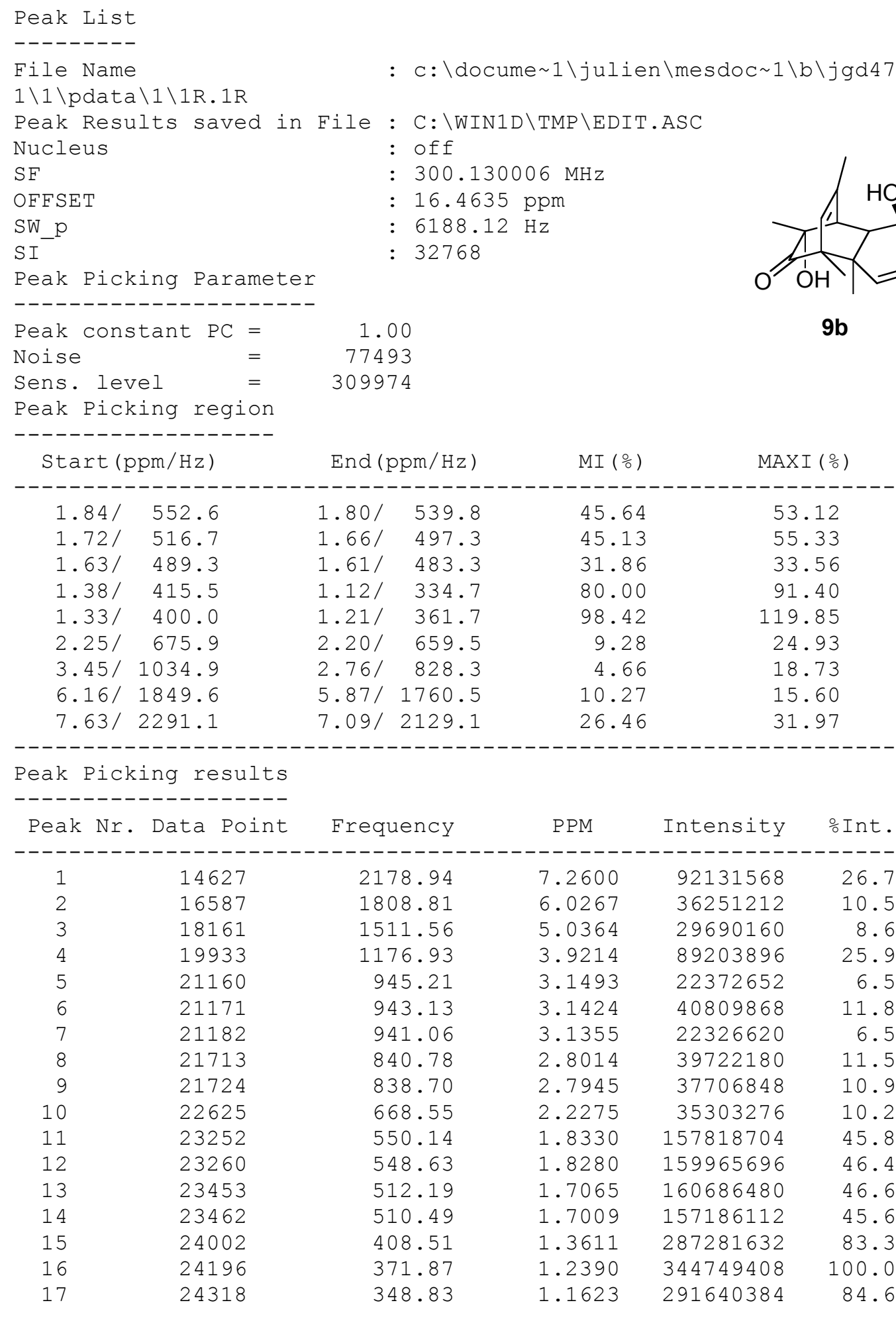


JGE33-1 - 071106 - DPX300 -

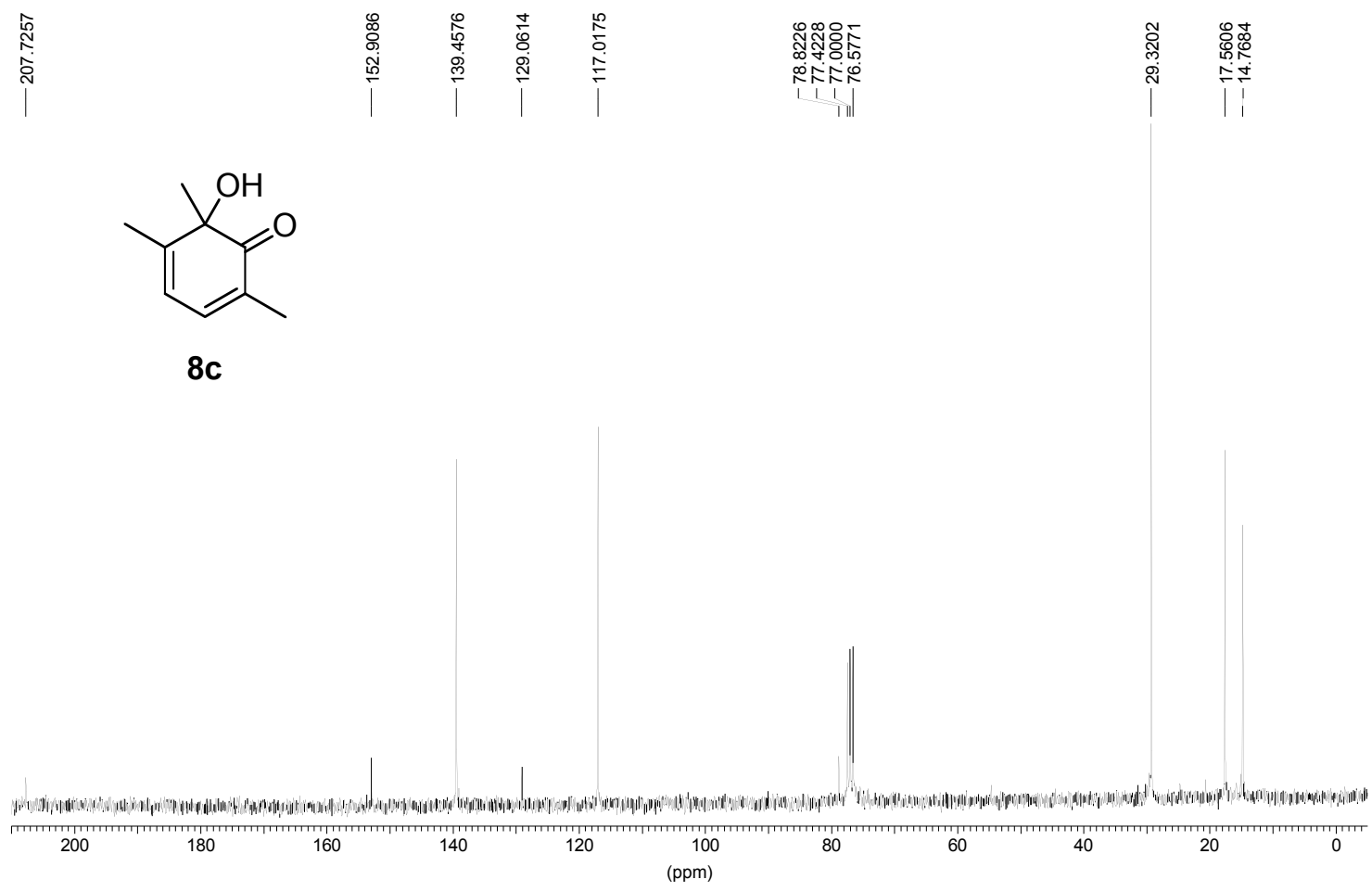

JGE33-1 - DPX300 - 071106 -

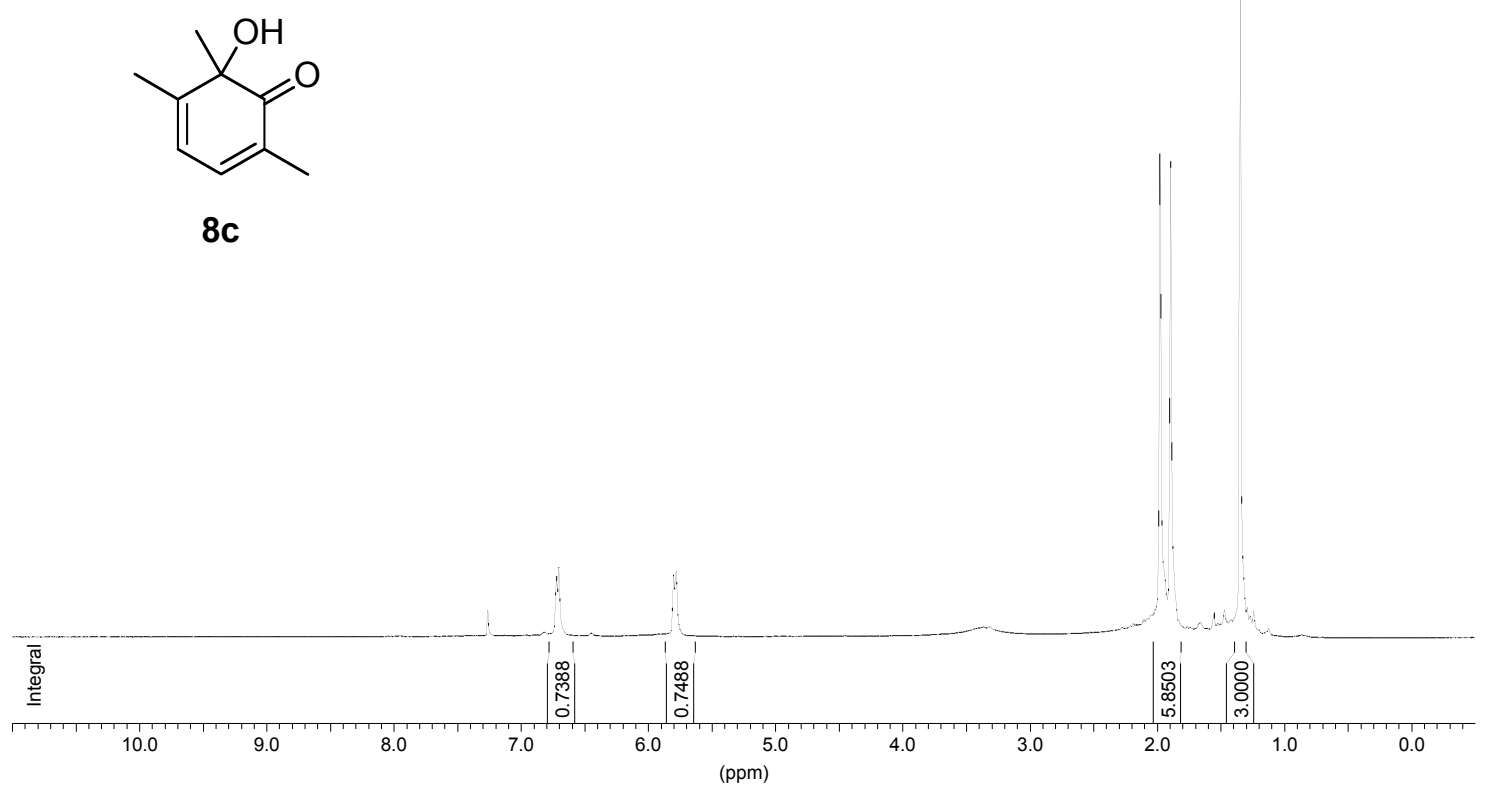




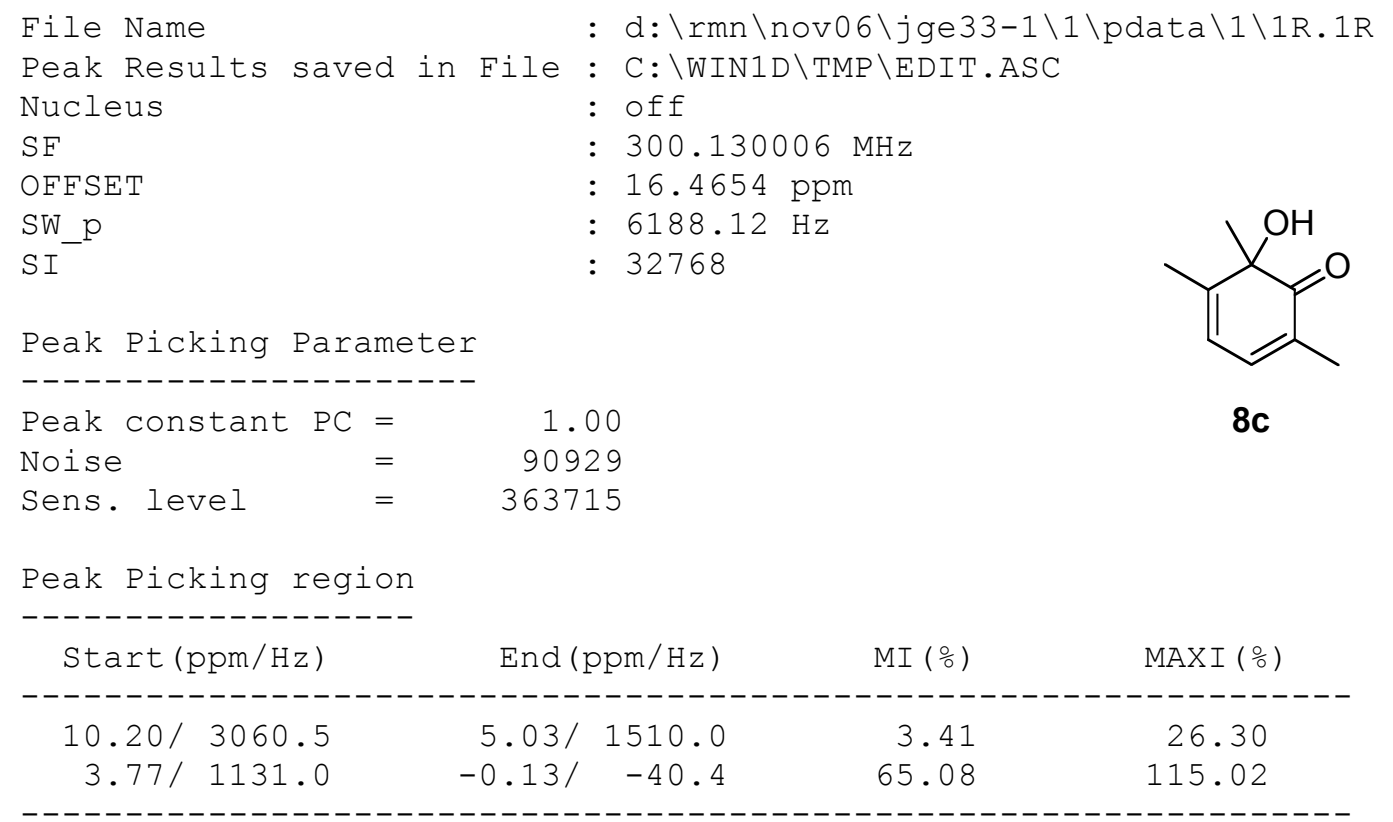

Peak Picking results

\begin{tabular}{|c|c|c|c|c|c|}
\hline Peak & Nr. Data Point & Frequency & PPM & Intensity & $\therefore$ Int. \\
\hline 1 & 14630 & 2178.94 & 7.2600 & 10420506 & 3.8 \\
\hline 2 & 15485 & 2017.48 & 6.7220 & 23411674 & 8.5 \\
\hline 3 & 15517 & 2011.44 & 6.7019 & 27573094 & 10.0 \\
\hline 4 & 16947 & 1741.39 & 5.8021 & 23651942 & 8.6 \\
\hline 5 & 16979 & 1735.34 & 5.7820 & 26332572 & 9.6 \\
\hline 6 & 23027 & 593.20 & 1.9765 & 187695456 & 68.1 \\
\hline 7 & 23161 & 567.90 & 1.8922 & 188485152 & 68.4 \\
\hline 8 & 24029 & 403.98 & 1.3460 & 275485024 & 100.0 \\
\hline
\end{tabular}


JGE33-3 - 300MHz -

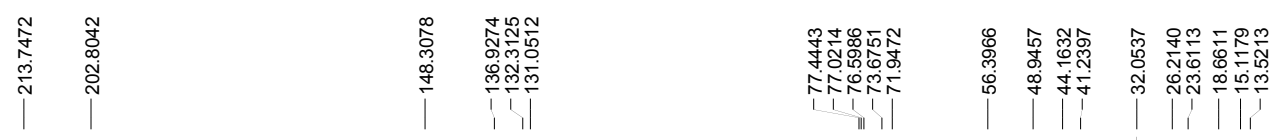

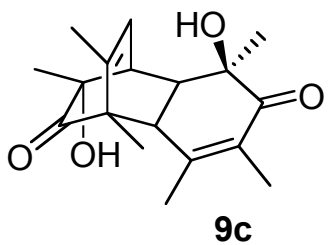

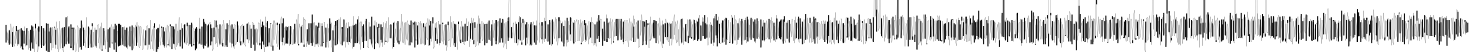

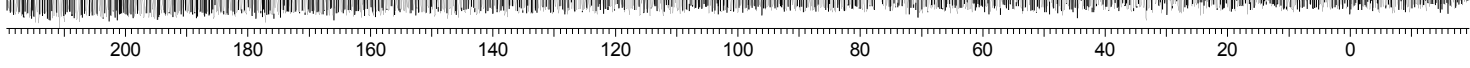
(ppm) JGE33-3 - 300MHz - 081206

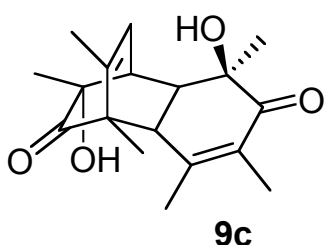

9c

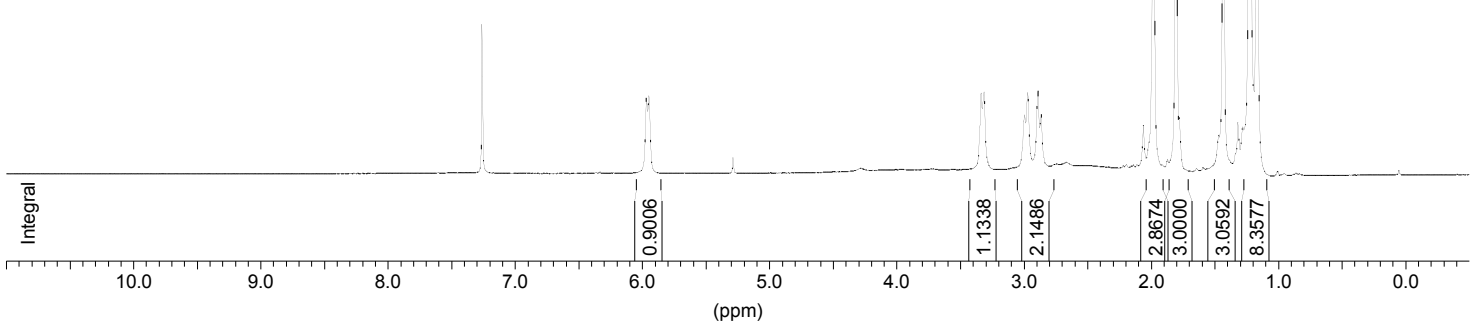




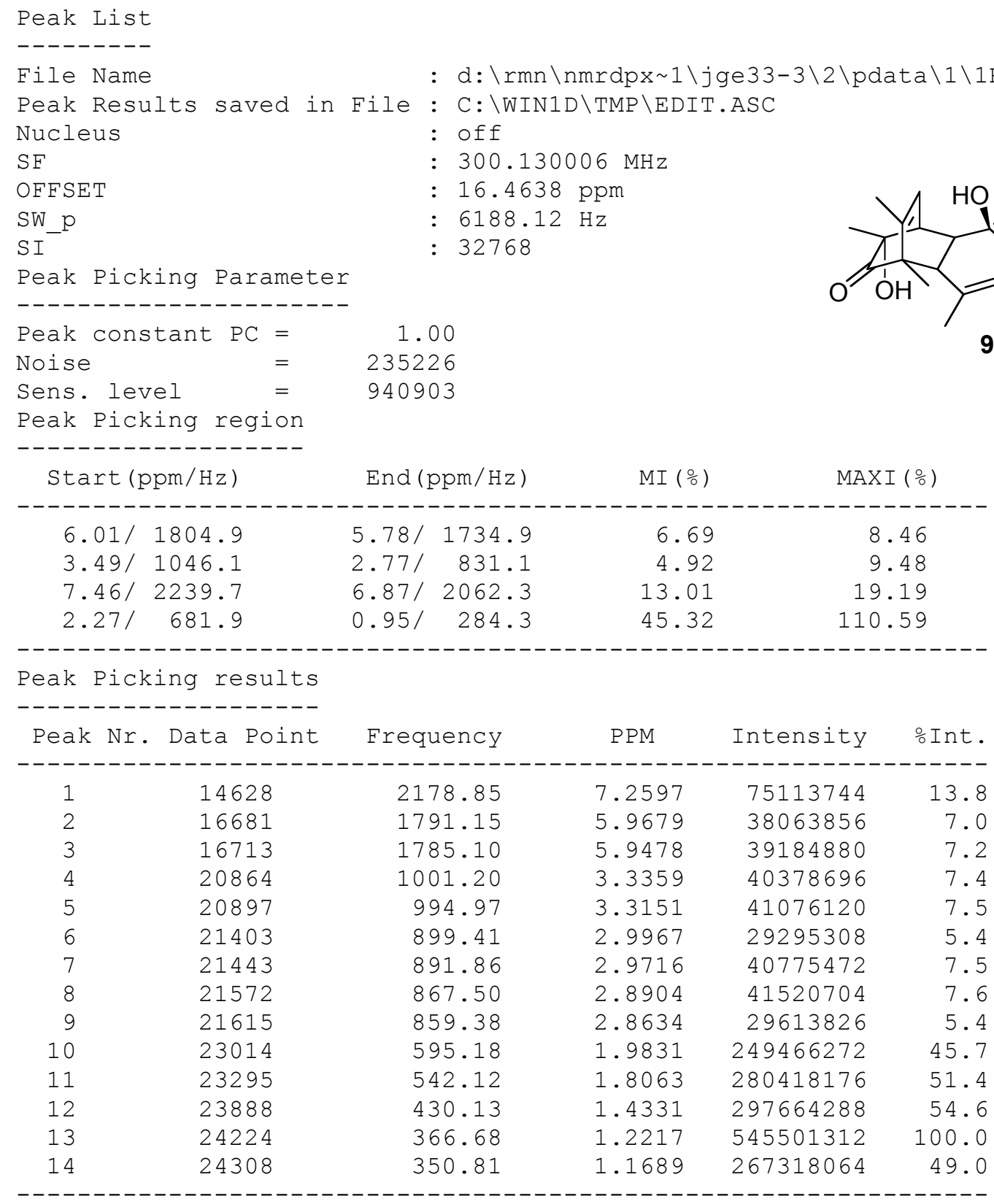




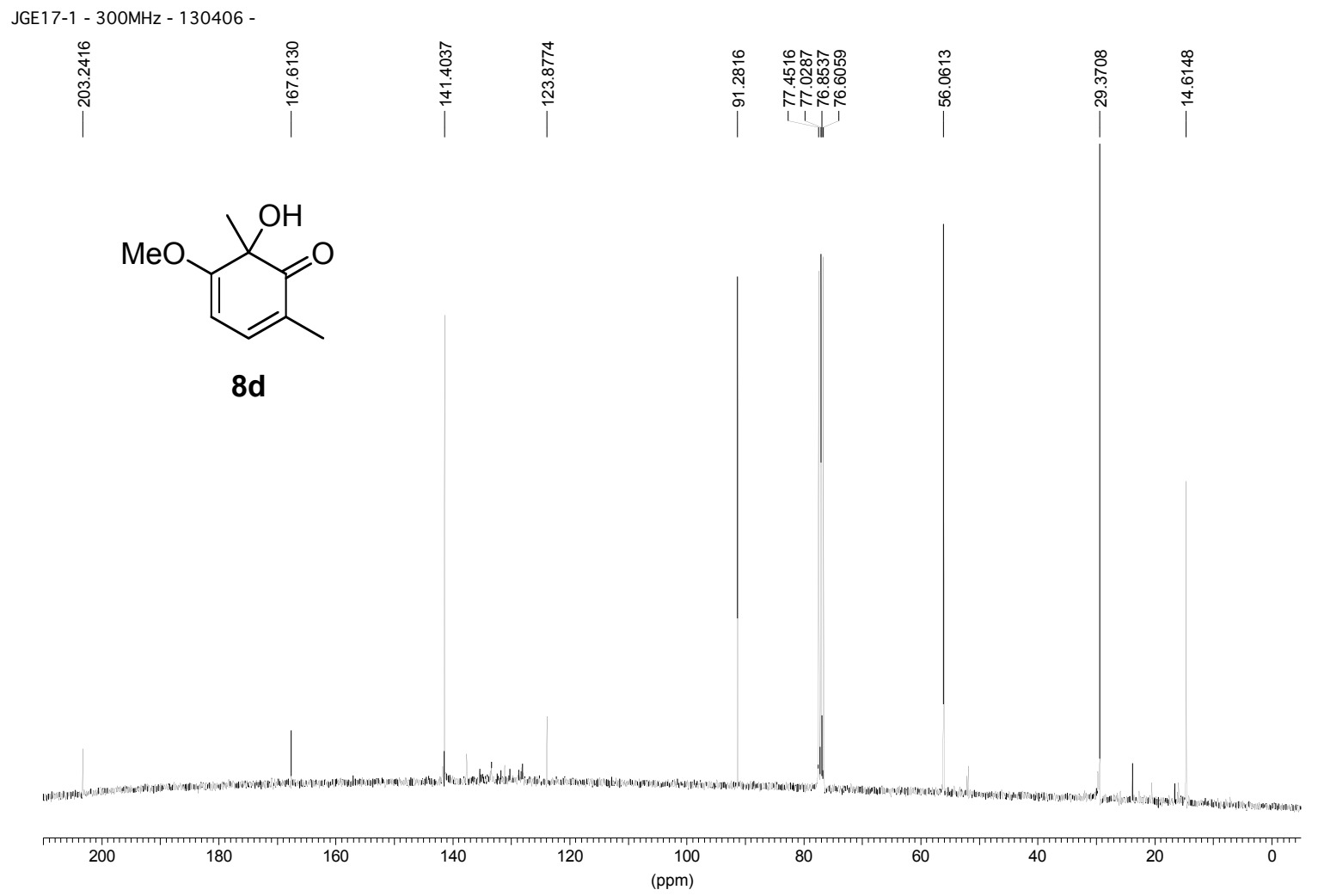

JGE17-1 - 130406 - 300MHz -

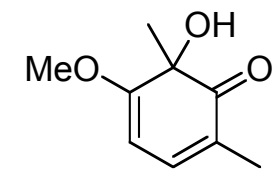

8d

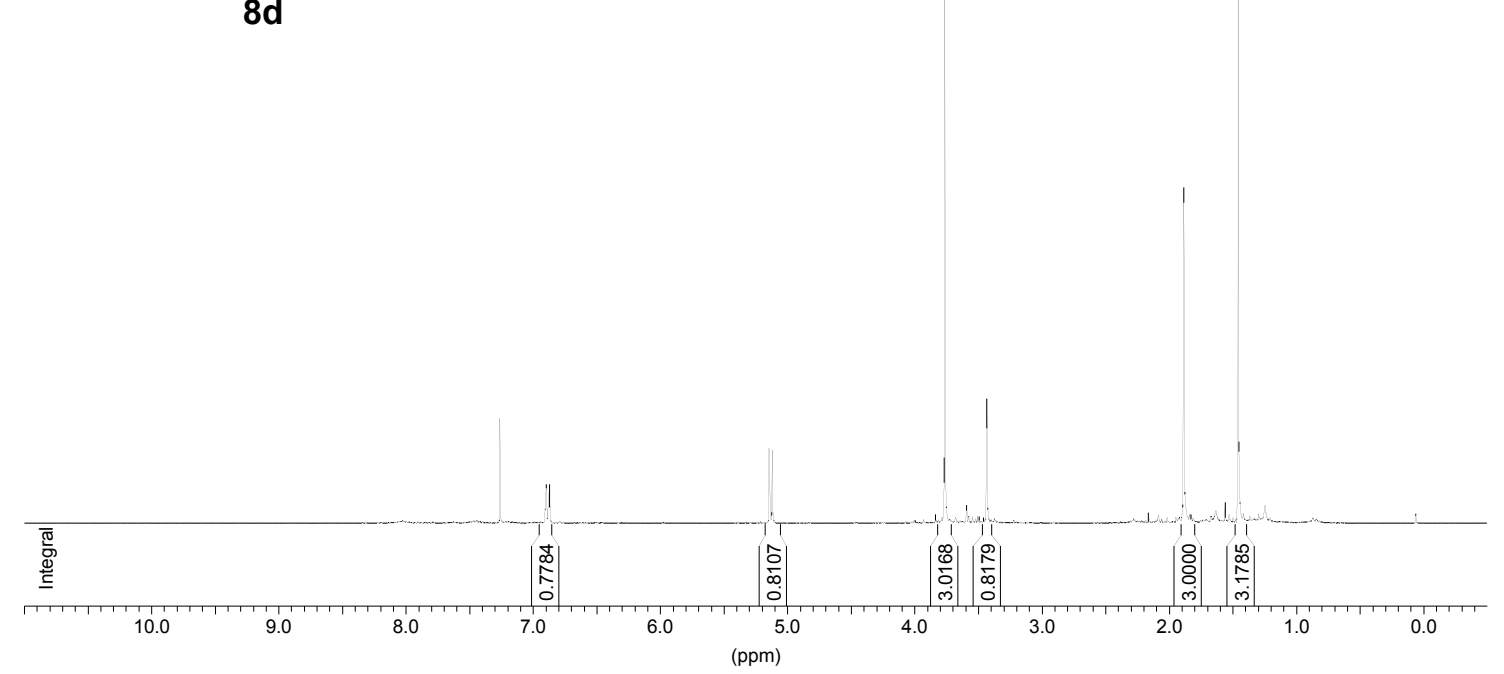




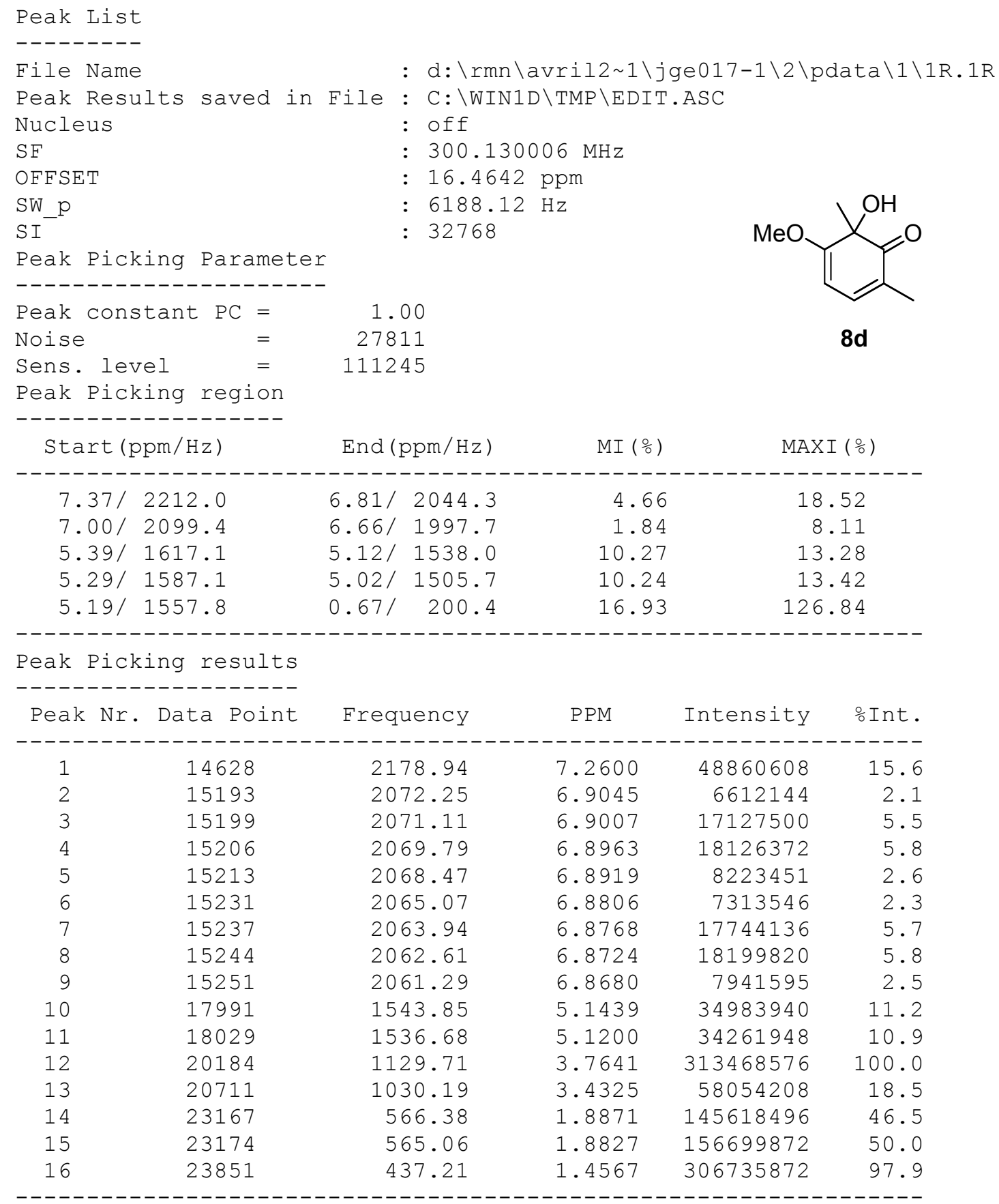


JGE17B-2 - DPX300 - 241106 -

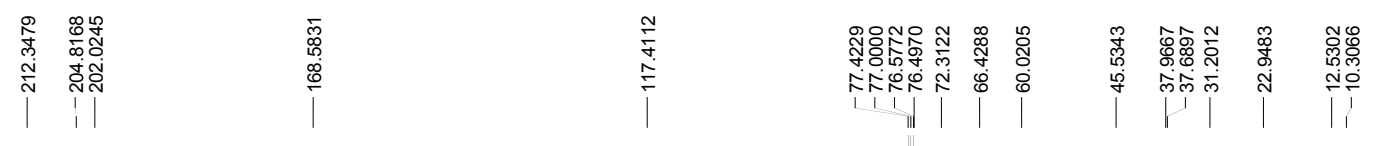

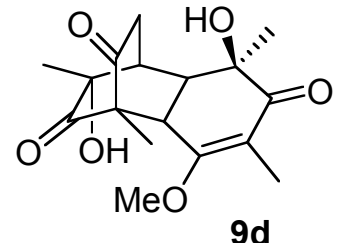

9d

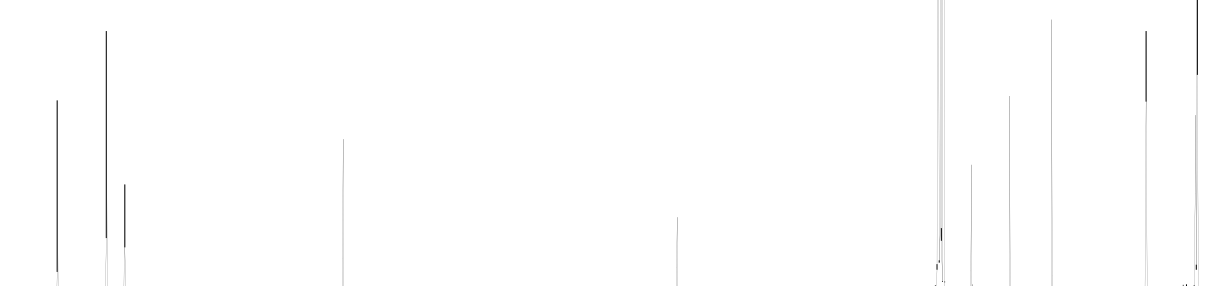

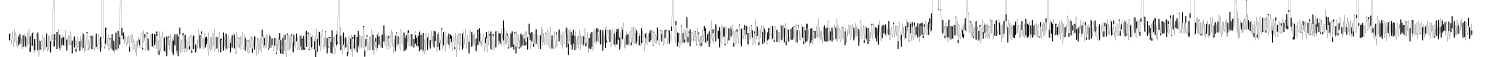

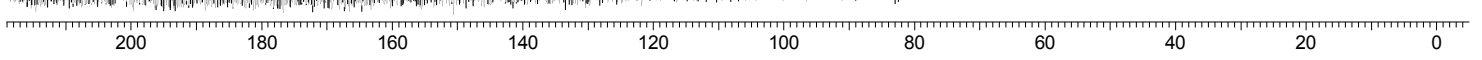
(ppm)
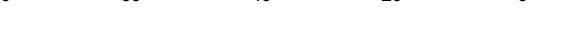

JGE17B-2 - 300MHz - $241106-$
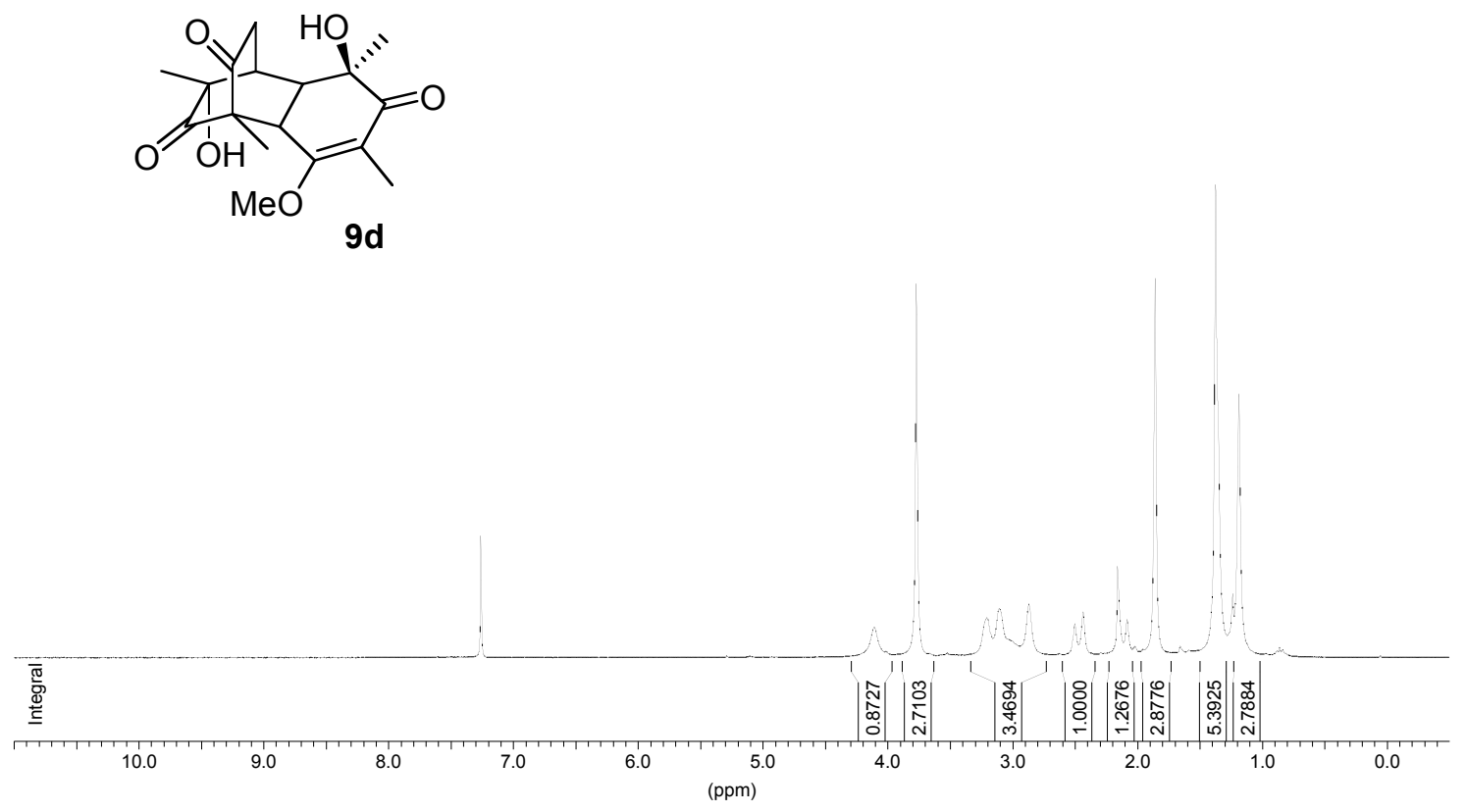


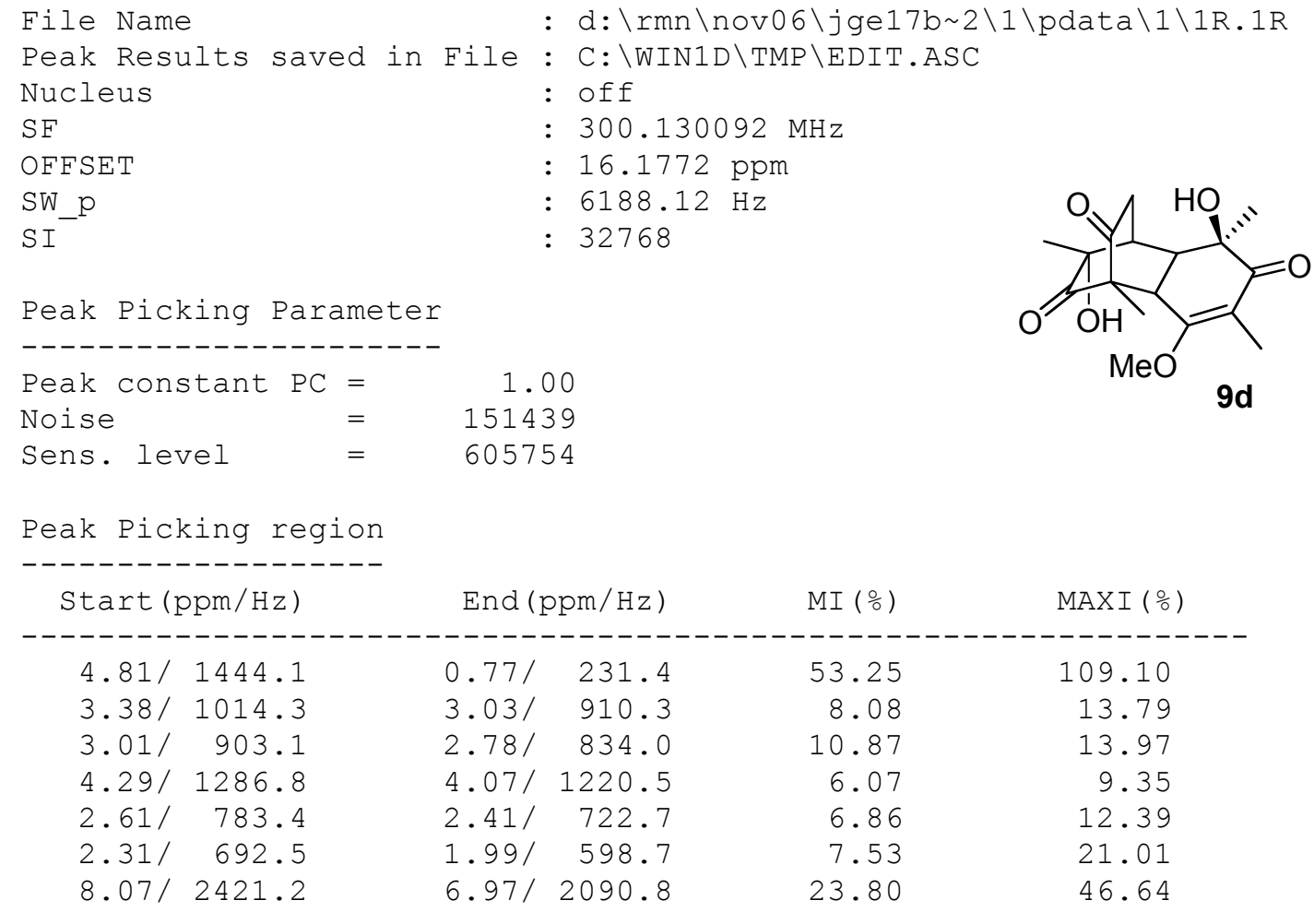

Peak Picking results

\begin{tabular}{|c|c|c|c|c|c|}
\hline Peak & Nr. Data Point & Frequency & PPM & Intensity & \%Int. \\
\hline-1 & ----------- & & -----1 & --------- & ----- \\
\hline 1 & 14172 & 2178.94 & 7.2600 & 76209064 & 25.7 \\
\hline 2 & 19180 & 1233.20 & 4.1089 & 19068232 & 6.4 \\
\hline 3 & 19718 & 1131.60 & 3.7704 & 234802512 & 79.1 \\
\hline 4 & 20618 & 961.64 & 3.2041 & 24944178 & 8.4 \\
\hline 5 & 20774 & 932.18 & 3.1059 & 30768208 & 10.4 \\
\hline 6 & 21152 & 860.80 & 2.8681 & 33345568 & 11.2 \\
\hline 7 & 21733 & 751.08 & 2.5025 & 21251498 & 7.2 \\
\hline 8 & 21840 & 730.87 & 2.4352 & 28756494 & 9.7 \\
\hline 9 & 22278 & 648.16 & 2.1596 & 57132168 & 19.2 \\
\hline 10 & 22401 & 624.93 & 2.0822 & 23918726 & 8.1 \\
\hline 11 & 22756 & 557.89 & 1.8588 & 238028016 & 80.2 \\
\hline 12 & 23527 & 412.29 & 1.3737 & 296919328 & 100.0 \\
\hline 13 & 23822 & 356.58 & 1.1881 & 165477232 & 55.7 \\
\hline
\end{tabular}


JGD52-2 - DPX400 - 050306 -
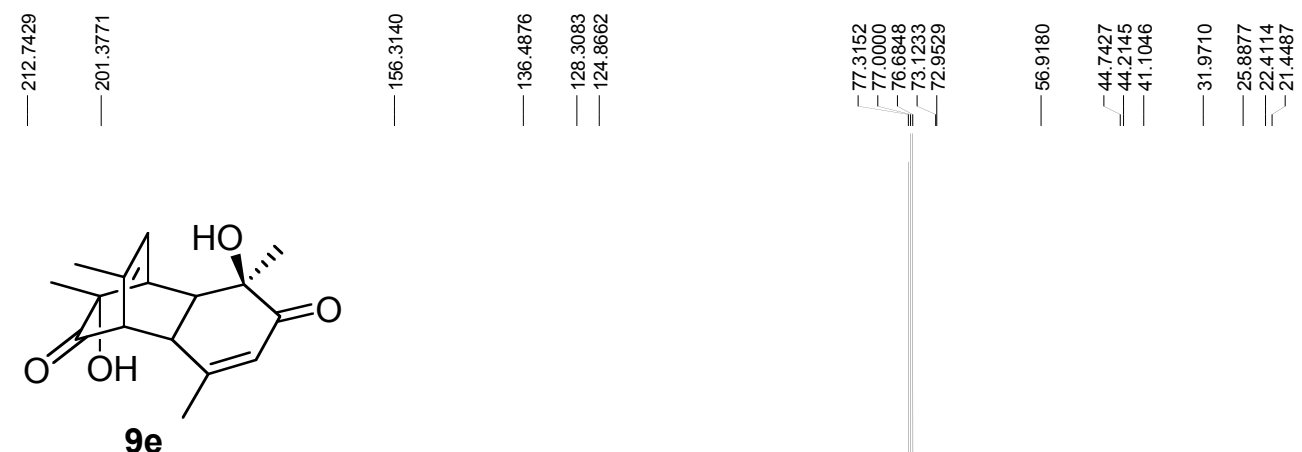

9e

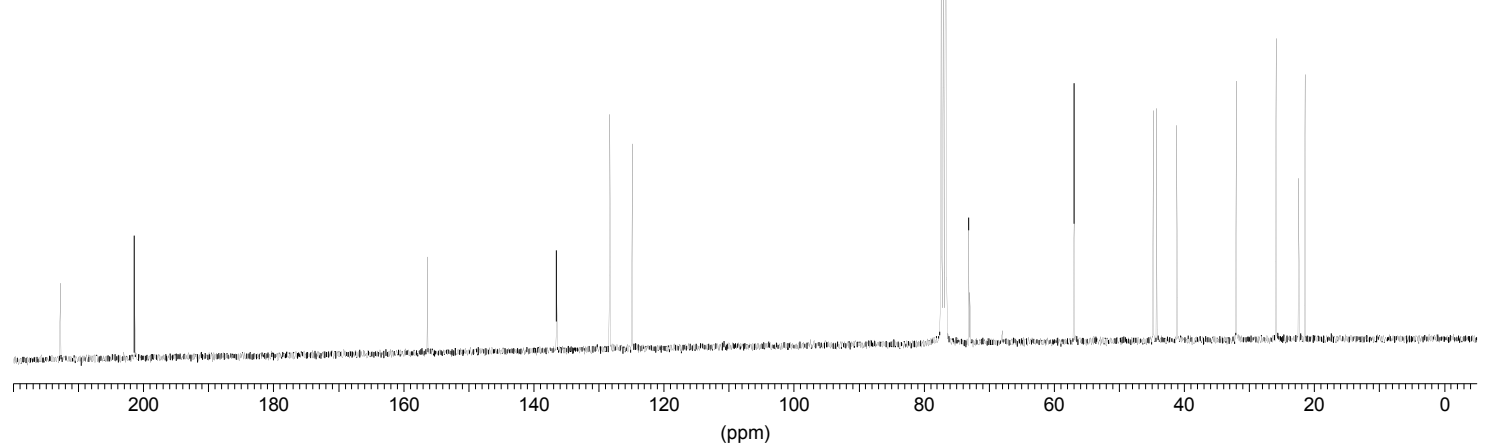

JGD52-2 - 300MHz - 240406 -

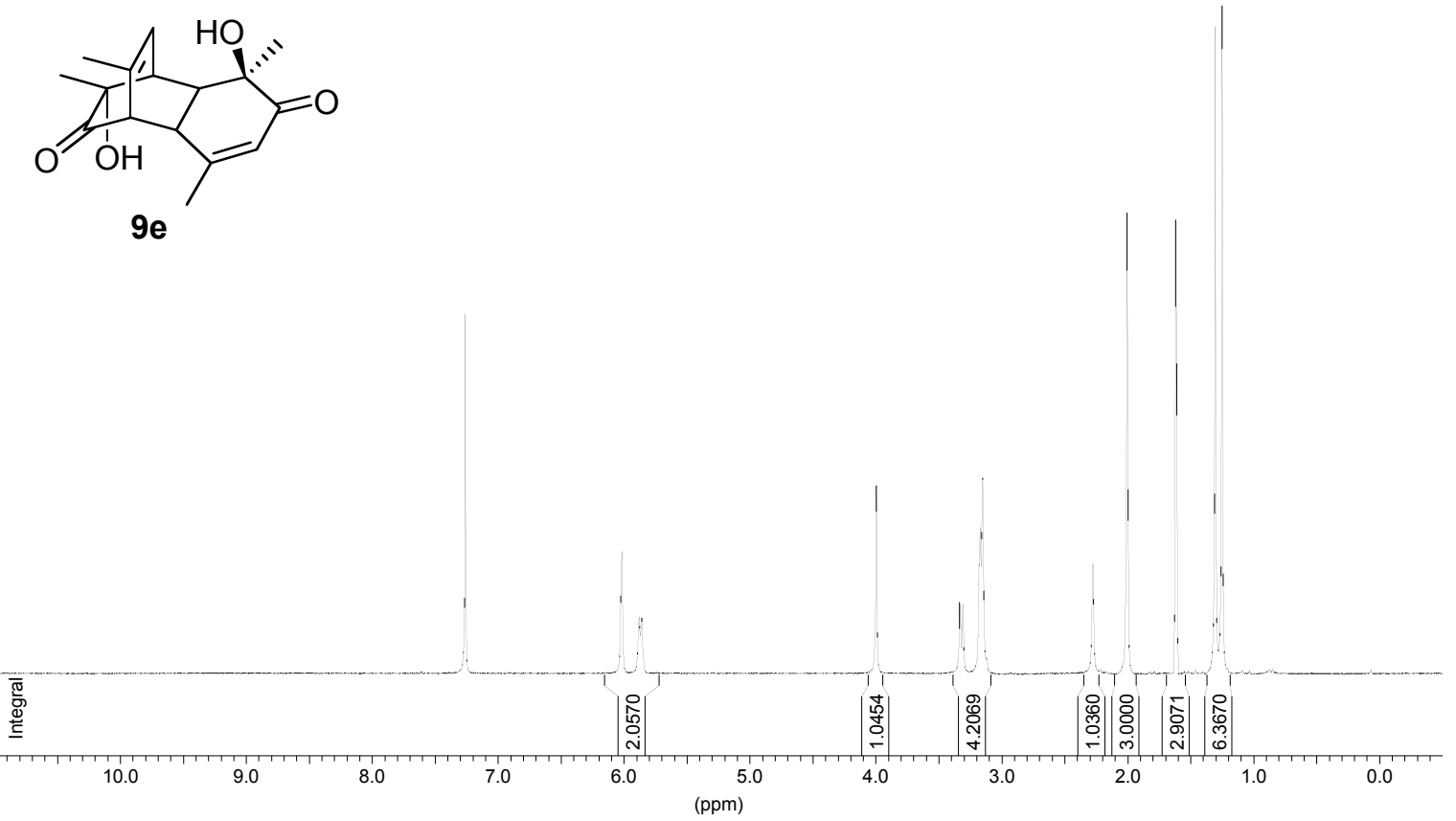




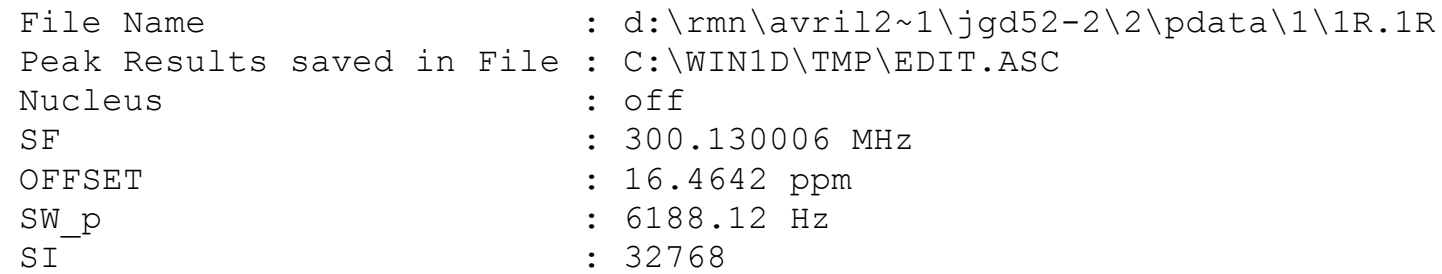

Peak Picking Parameter

$\begin{array}{llr}\text { Peak constant PC } & = & 1.00 \\ \text { Noise } & = & 115425 \\ \text { Sens. level } & = & 461701\end{array}$

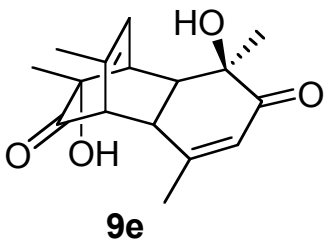

Peak Picking region

\begin{tabular}{|c|c|c|c|c|}
\hline Start (ppm/Hz) & End (ppm/Hz) & MI ( $\%)$ & \multicolumn{2}{|c|}{ MAXI (\%) } \\
\hline \multicolumn{5}{|c|}{----------- - - - - - - - - - - - - - - - - - - - - - - - - - - - - - - - - - - - - - - - - - - - - - } \\
\hline $3.40 / 1021.7$ & $3.29 / 987.5$ & 8.91 & \multicolumn{2}{|c|}{12.16} \\
\hline $7.82 / 2348.3$ & $5.56 / 1667.9$ & 6.60 & \multicolumn{2}{|c|}{64.41} \\
\hline $2.56 / 768.6$ & $2.06 / 618.7$ & 15.63 & \multicolumn{2}{|c|}{24.83} \\
\hline $2.32 / 695.6$ & $1.45 /$ & 66.15 & \multicolumn{2}{|c|}{76.04} \\
\hline $1.72 / 515.0$ & $0.55 / 165.3$ & 92.02 & \multicolumn{2}{|c|}{103.83} \\
\hline $4.80 / 1441.3$ & $3.79 / 1137.6$ & 28.48 & \multicolumn{2}{|c|}{61.12} \\
\hline $4.70 / 1410.5$ & $3.78 / 1133.9$ & 27.09 & \multicolumn{2}{|c|}{60.77} \\
\hline \multicolumn{5}{|c|}{ 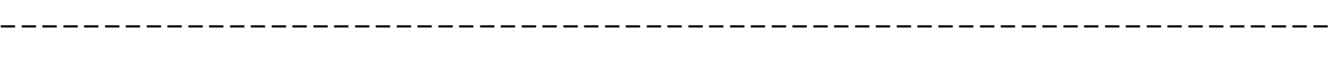 } \\
\hline \multicolumn{5}{|l|}{ Peak Picking results } \\
\hline Peak Nr. Data Point & Frequency & PPM & Intensity & $\circ \operatorname{Int}$. \\
\hline 14628 & 2178.94 & 7.2600 & 154825552 & 53.8 \\
\hline 16603 & 1805.97 & 6.0173 & 52506044 & 18.2 \\
\hline 16822 & 1764.62 & 5.8795 & 24401028 & 8.5 \\
\hline 16858 & 1757.82 & 5.8569 & 23834774 & 8.3 \\
\hline 19815 & 1199.40 & 3.9963 & 80981936 & 28.1 \\
\hline 20868 & 1000.54 & 3.3337 & 30579236 & 10.6 \\
\hline 20875 & 999.22 & 3.3293 & 30421046 & 10.6 \\
\hline 20904 & 993.74 & 3.3110 & 29702136 & 10.3 \\
\hline 20911 & 992.42 & 3.3066 & 28719682 & 10.0 \\
\hline 22548 & 683.28 & 2.2766 & 47104736 & 16.4 \\
\hline 22979 & 601.89 & 2.0054 & 198407904 & 68.9 \\
\hline 22984 & 600.94 & 2.0023 & 196714720 & 68.3 \\
\hline 23596 & 485.37 & 1.6172 & 195402176 & 67.9 \\
\hline 23603 & 484.05 & 1.6128 & 192196544 & 66.8 \\
\hline 24093 & 391.51 & 1.3045 & 278892128 & 96.9 \\
\hline 24182 & 374.71 & 1.2485 & 287810528 & 100.0 \\
\hline
\end{tabular}


AOA44 - jv171f.105 - 250MHz -

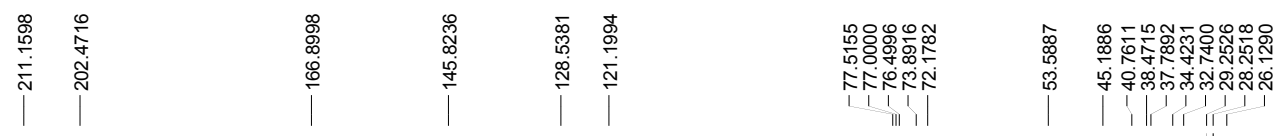

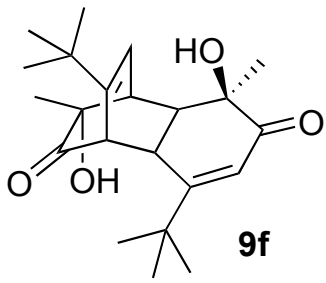

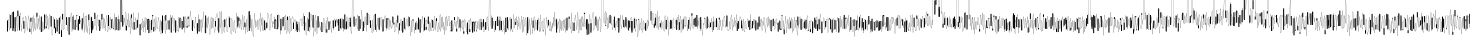

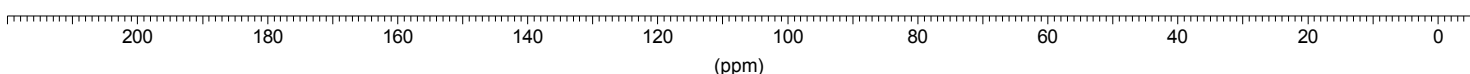

AOA44/CDCl3/popo
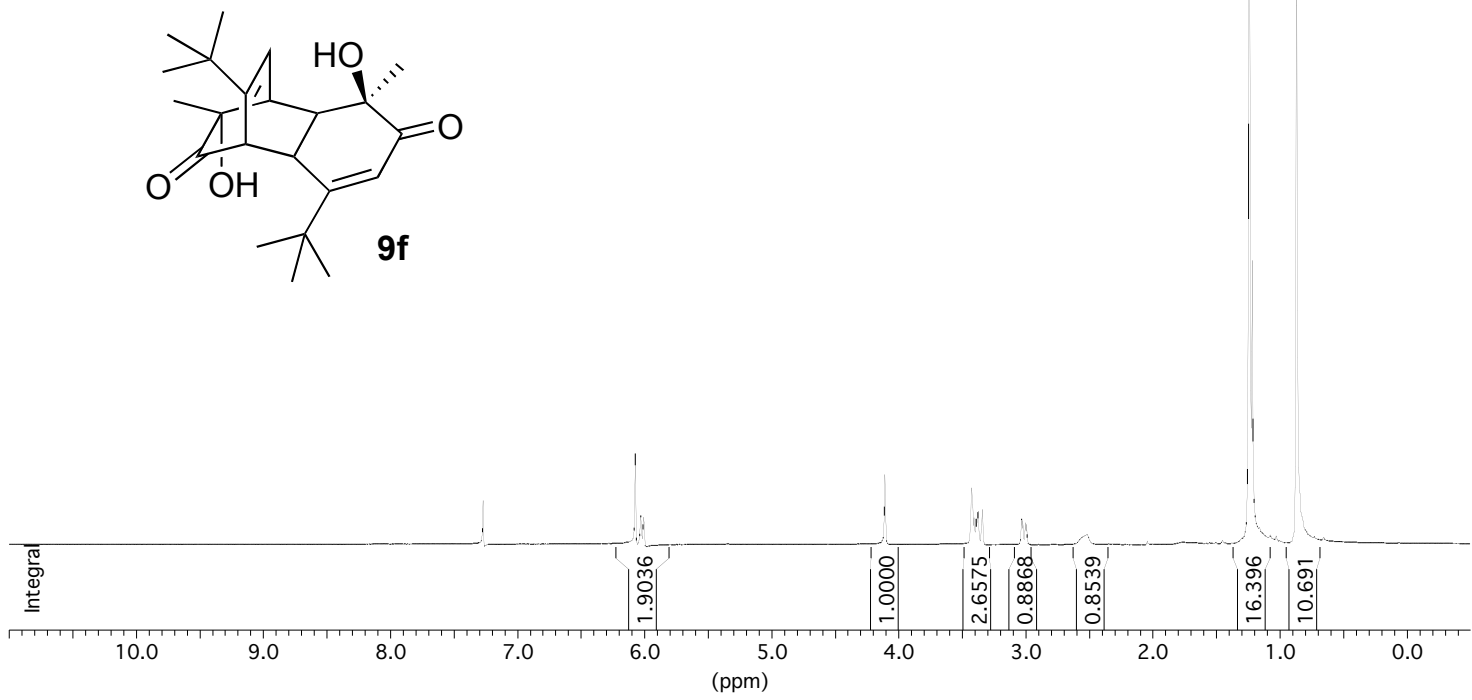
Peak List

File Name $\quad$ : d: Inmr\aoalaoa44\17010318\001001.1R

Peak Results saved in File : $: \backslash W I N 1 D \backslash T M P \backslash E D I T . A S C$

Nucleus

: off

SF $\quad: 300.130003 \mathrm{MHz}$

OFFSET $\quad: 19.7730 \mathrm{ppm}$

SW_p

SI 16384

Peak Picking Parameter

Peak constant $\mathrm{PC}=1.00$

Noise $=10716$

Sens. level $=42862$

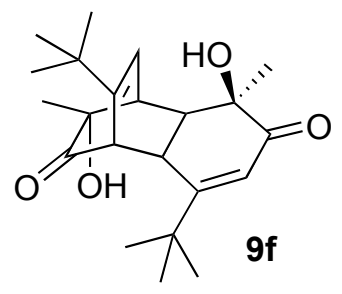

Peak Picking region

\begin{tabular}{lccc} 
Start(ppm/Hz) & End(ppm/Hz) & MI(\%) & MAXI(\%) \\
\hline$-11 / 634.2$ & $0.68 / 205.5$ & 84.14 & 126.68 \\
$2.66 / 797.7$ & $2.42 / 725.4$ & 0.66 & 11.36 \\
$4.19 / 1258.5$ & $3.02 / 907.4$ & 9.08 & 67.54 \\
$3.06 / 919.0$ & $2.98 / 894.9$ & 2.37 & 3.70 \\
$3.48 / 1043.8$ & $3.41 / 1024.5$ & 6.77 & 9.63 \\
$3.41 / 1023.4$ & $3.39 / 1016.6$ & 2.98 & 3.84 \\
$3.39 / 1018.5$ & $3.32 / 997.8$ & 3.99 & 5.26 \\
$4.70 / 1411.5$ & $3.92 / 1177.5$ & 6.15 & 17.71 \\
$1.23 / 367.8$ & $1.21 / 363.3$ & 33.13 & 42.76
\end{tabular}

Peak Picking results

Peak Nr. Data Point Frequency PPM Intensity \%Int.

\begin{tabular}{rrrrrr}
1 & 10915 & 1822.14 & 6.0712 & 36992452 & 11.2 \\
2 & 10948 & 1809.71 & 6.0297 & 11953892 & 3.6 \\
3 & 10967 & 1802.55 & 6.0059 & 11130506 & 3.4 \\
4 & 12479 & 1232.89 & 4.1078 & 28641400 & 8.7 \\
5 & 13022 & 1028.31 & 3.4262 & 23170818 & 7.0 \\
6 & 13039 & 1021.90 & 3.4049 & 10918567 & 3.3 \\
7 & 13050 & 1017.76 & 3.3911 & 10541285 & 3.2 \\
8 & 13064 & 1012.48 & 3.3735 & 13641368 & 4.1 \\
9 & 13088 & 1003.44 & 3.3433 & 14369223 & 4.4 \\
10 & 13336 & 910.00 & 3.0320 & 10556803 & 3.2 \\
11 & 13344 & 906.99 & 3.0220 & 9993312 & 3.0 \\
12 & 13360 & 900.96 & 3.0019 & 8991985 & 2.7 \\
13 & 13368 & 897.95 & 2.9919 & 8278457 & 2.5 \\
14 & 13746 & 755.53 & 2.5173 & 4196589 & 1.3 \\
15 & 14763 & 372.37 & 1.2407 & 329254848 & 100.0 \\
16 & 14781 & 365.58 & 1.2181 & 115452784 & 35.1 \\
17 & 15060 & 260.47 & 0.8679 & 286738240 & 87.1 \\
\hline
\end{tabular}


JGC266-4 - DPX400 - 050805 -

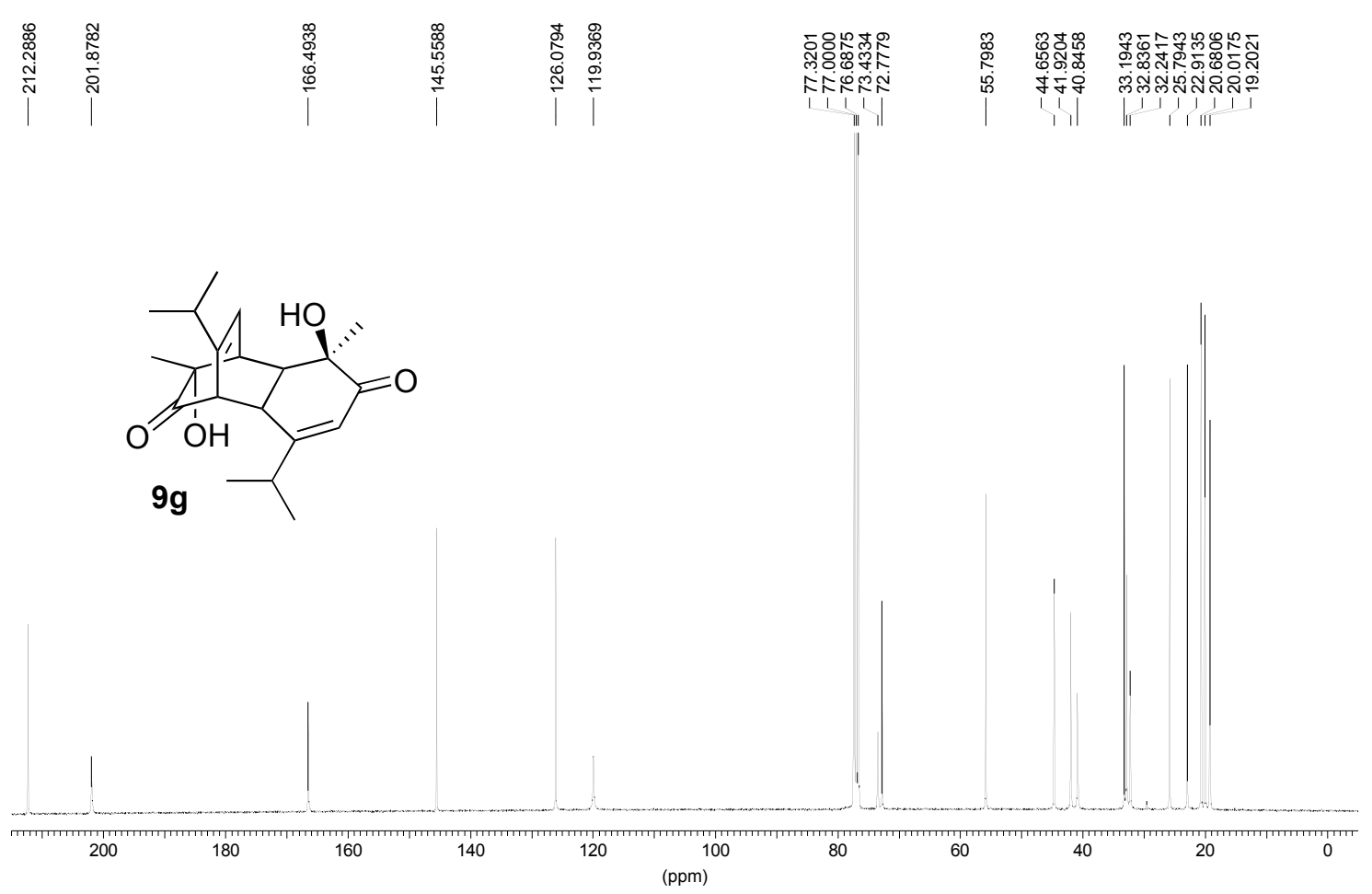

JGE13A-2-recrist - 300MHz - 220406 -

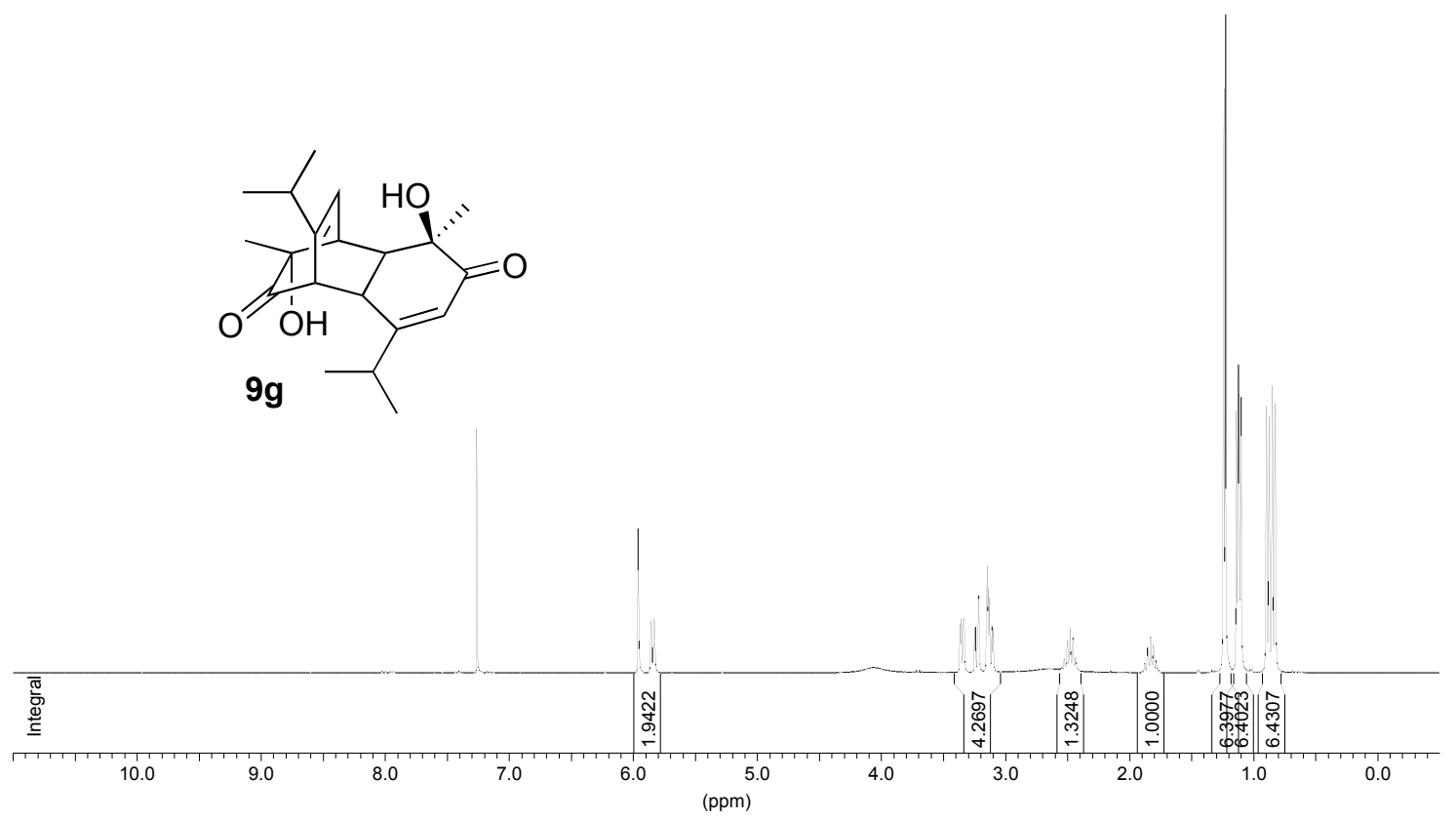




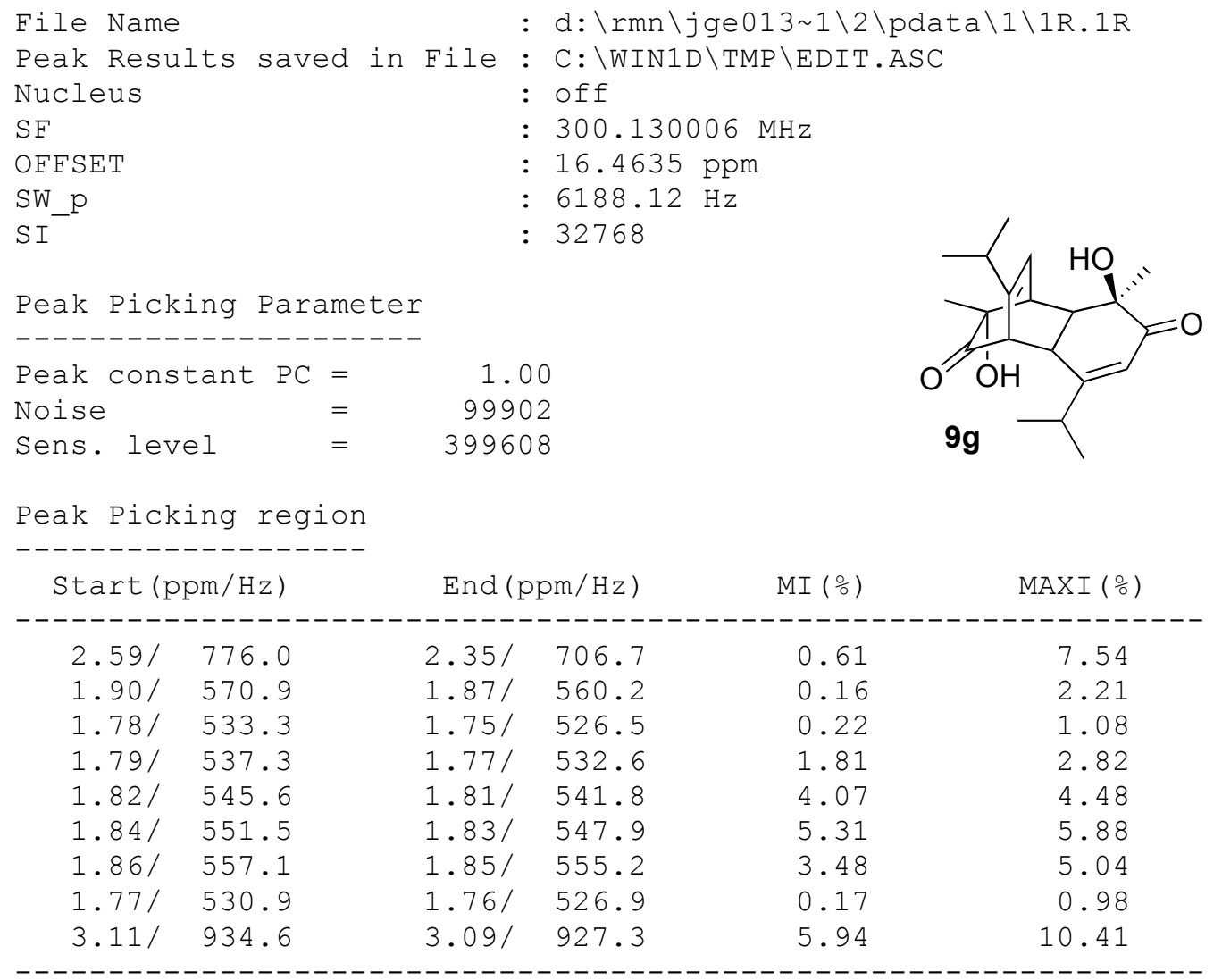

Peak Picking results

\begin{tabular}{|c|c|c|c|c|c|}
\hline Peak & Nr. Data Point & Frequency & PPM & Intensity & $\%$ Int. \\
\hline----- & -------------- & ----------- & ------- & ---------- & ----- \\
\hline 1 & 14627 & 2178.94 & 7.2600 & 118798960 & 37.1 \\
\hline 2 & 16695 & 1788.41 & 5.9588 & 69934056 & 21.8 \\
\hline 3 & 16862 & 1756.87 & 5.8537 & 25119614 & 7.8 \\
\hline 4 & 16898 & 1750.07 & 5.8311 & 26192540 & 8.2 \\
\hline 5 & 20816 & 1010.17 & 3.3658 & 18879232 & 5.9 \\
\hline 6 & 20821 & 1009.23 & 3.3626 & 25221260 & 7.9 \\
\hline 7 & 20828 & 1007.91 & 3.3582 & 22982432 & 7.2 \\
\hline 8 & 20834 & 1006.77 & 3.3545 & 26291602 & 8.2 \\
\hline 9 & 20852 & 1003.38 & 3.3431 & 20041456 & 6.3 \\
\hline 10 & 20857 & 1002.43 & 3.3400 & 25194152 & 7.9 \\
\hline 11 & 20865 & 1000.92 & 3.3350 & 23107572 & 7.2 \\
\hline 12 & 20870 & 999.98 & 3.3318 & 26338258 & 8.2 \\
\hline 13 & 21002 & 975.05 & 3.2488 & 18611726 & 5.8 \\
\hline 14 & 21012 & 973.16 & 3.2425 & 22176124 & 6.9 \\
\hline 15 & 21048 & 966.36 & 3.2198 & 30863660 & 9.6 \\
\hline 16 & 21058 & 964.47 & 3.2135 & 37362020 & 11.7 \\
\hline 17 & 21181 & 941.24 & 3.1361 & 41119756 & 12.8 \\
\hline 18 & 21194 & 938.79 & 3.1279 & 36483872 & 11.4 \\
\hline 19 & 21227 & 932.56 & 3.1072 & 22358040 & 7.0 \\
\hline 20 & 21240 & 930.10 & 3.0990 & 22000848 & 6.9 \\
\hline 21 & 22119 & 764.11 & 2.5459 & 2412906 & 0.8 \\
\hline 22 & 22154 & 757.50 & 2.5239 & 6916284 & 2.2 \\
\hline 23 & 22190 & 750.70 & 2.5012 & 15946376 & 5.0 \\
\hline 24 & 22226 & 743.90 & 2.4786 & 21626308 & 6.8 \\
\hline
\end{tabular}




$\begin{array}{rrrrrr}25 & 22261 & 737.29 & 2.4566 & 16956178 & 5.3 \\ 26 & 22297 & 730.49 & 2.4339 & 7522006 & 2.3 \\ 27 & 22333 & 723.69 & 2.4113 & 1976065 & 0.6 \\ 28 & 23149 & 569.60 & 1.8978 & 683631 & 0.2 \\ 29 & 23185 & 562.80 & 1.8752 & 4528644 & 1.4 \\ 30 & 23221 & 556.00 & 1.8525 & 12173032 & 3.8 \\ 31 & 23257 & 549.20 & 1.8299 & 17320846 & 5.4 \\ 32 & 23293 & 542.40 & 1.8072 & 13733128 & 4.3 \\ 33 & 23329 & 535.60 & 1.7846 & 5845228 & 1.8 \\ 34 & 23365 & 528.80 & 1.7619 & 1077140 & 0.3 \\ 35 & 24197 & 371.68 & 1.2384 & 284078656 & 88.7 \\ 36 & 24218 & 367.72 & 1.2252 & 320151872 & 100.0 \\ 37 & 24359 & 341.09 & 1.1365 & 127368280 & 39.8 \\ 38 & 24381 & 336.94 & 1.1226 & 149856192 & 46.8 \\ 39 & 24394 & 334.48 & 1.1145 & 135602400 & 42.4 \\ 40 & 24418 & 329.95 & 1.0994 & 134062128 & 41.9 \\ 41 & 24743 & 268.57 & 0.8949 & 129483536 & 40.4 \\ 42 & 24778 & 261.96 & 0.8728 & 125000136 & 39.0 \\ 43 & 24819 & 254.22 & 0.8470 & 139360176 & 43.5 \\ 44 & 24855 & 247.42 & 0.8244 & 130770768 & 40.8 \\ -----------------------------------------------------\end{array}$


JGE15-1-1 - 260406 - 300MHz -

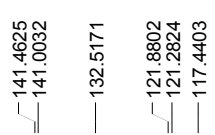

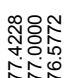

器器 :

Kरำ

กิ ลู่

$10 \mathrm{~g}$<smiles>Cc1ccc(C(C)C)c(O)c1O</smiles>

JGE15-1-1 - 260406 - 300MHz

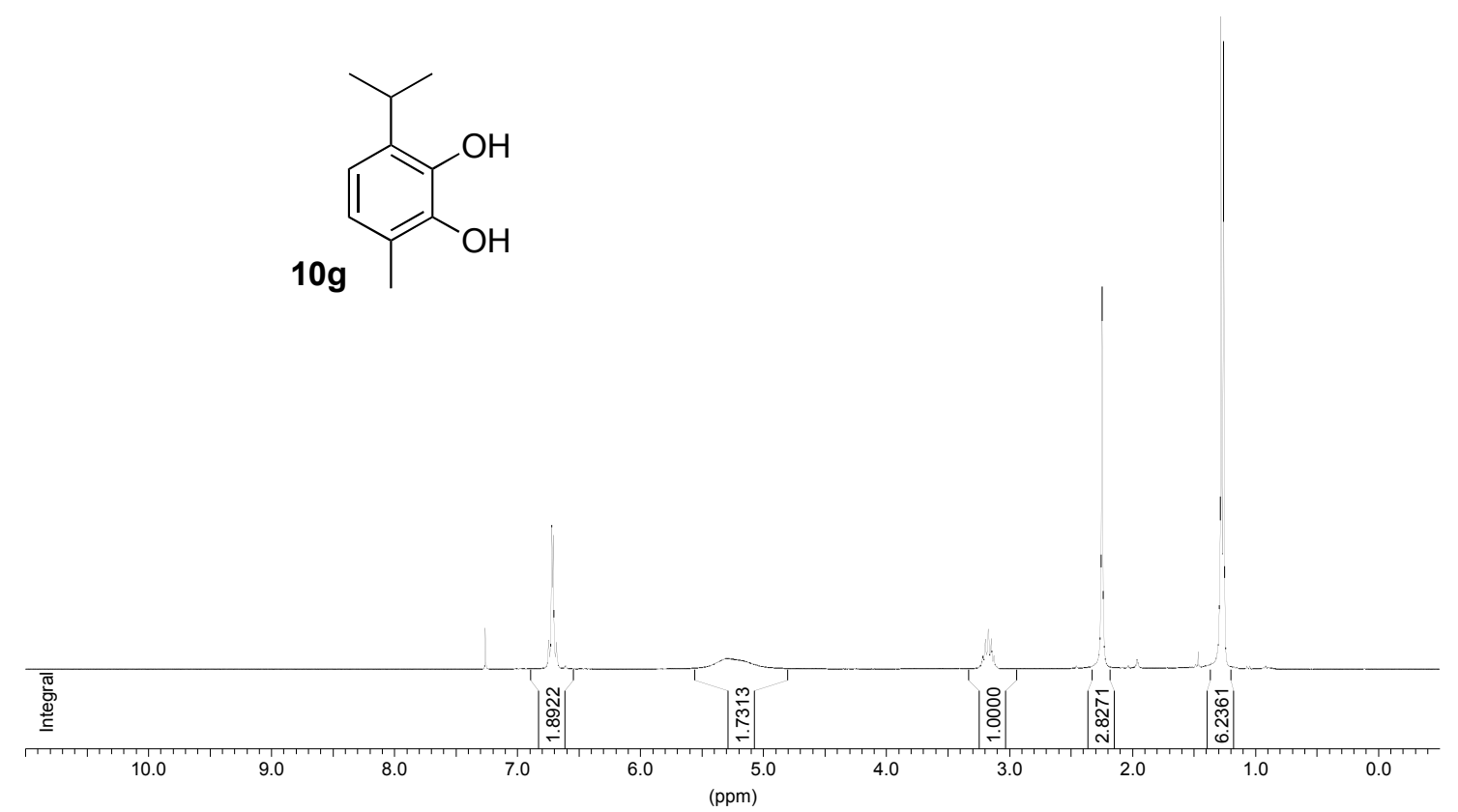


File Name

Peak Results saved in File: Nucleus

SF

OFFSET

SW_P

$\mathrm{SI}^{-}$

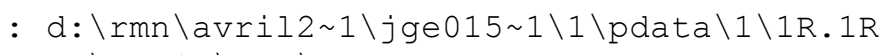

: off

: $300.130006 \mathrm{MHz}$

: $16.4642 \mathrm{ppm}$

: $6188.12 \mathrm{~Hz}$

: 32768

Peak Picking Parameter

$\begin{array}{rrr}\text { Peak constant PC } & = & 1.00 \\ \text { Noise } & = & 60060 \\ \text { Sens. level } & = & 240242\end{array}$

Sens. level = 240242

$10 \mathrm{~g}$<smiles>Cc1ccc(C(C)C)c(O)c1O</smiles>

Peak Picking region

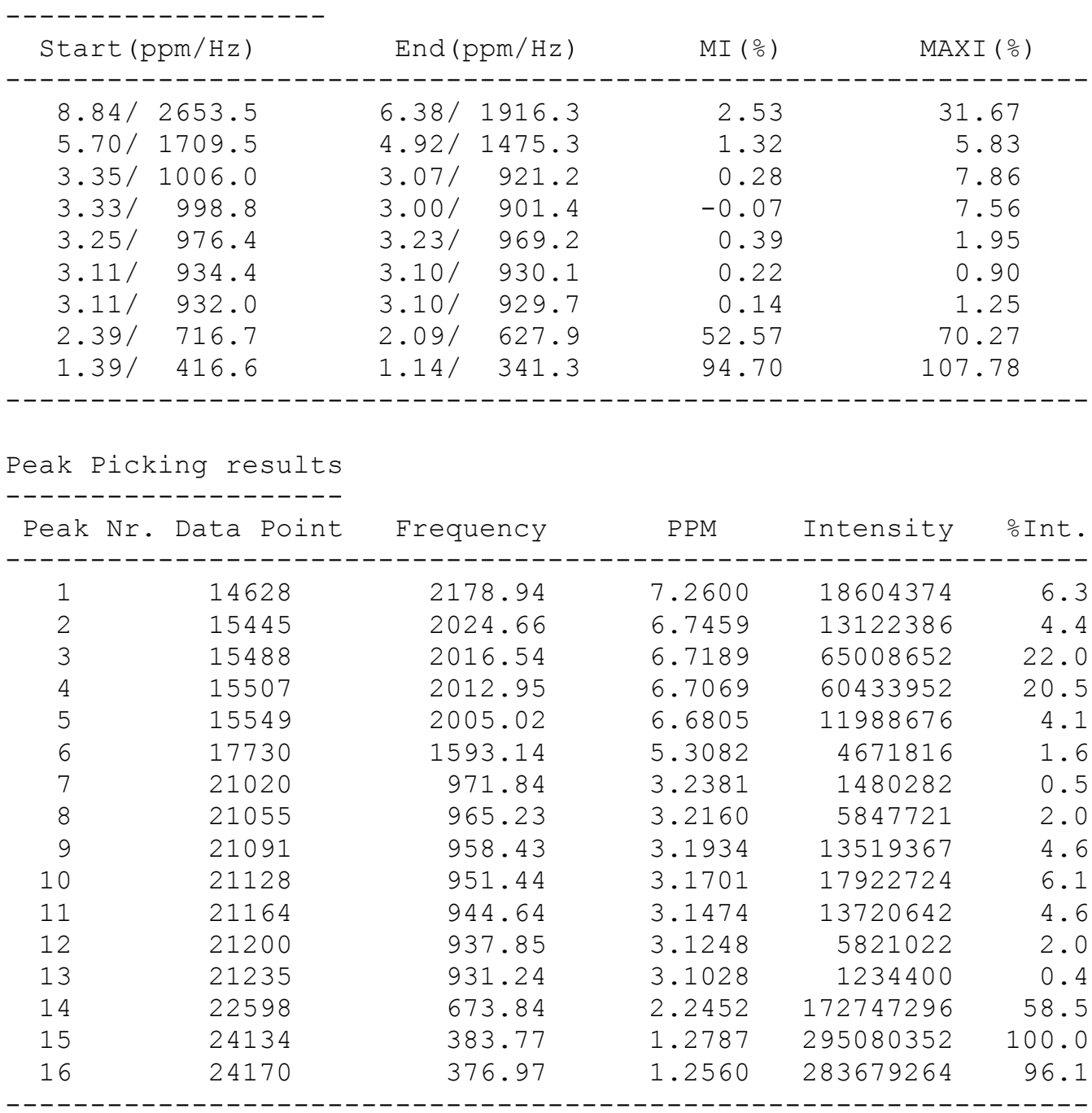


JGC266-2 - DPX400 - 200306 -

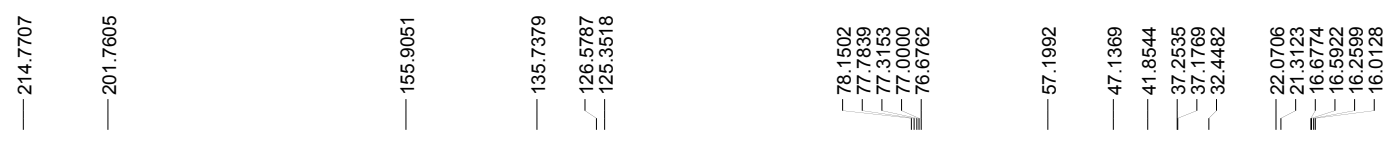

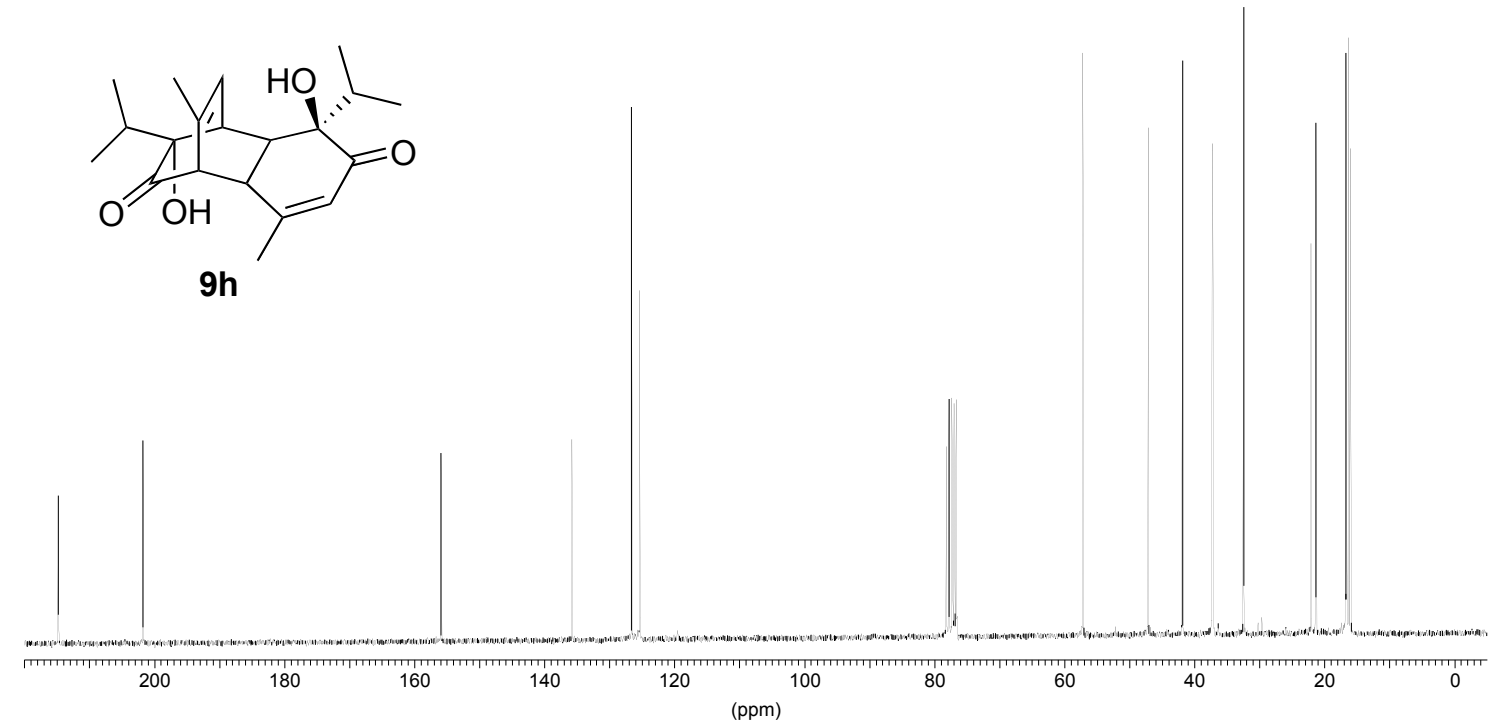

JGC266-2 - DPX400 - 200306 -

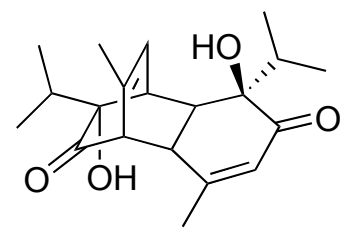

9h

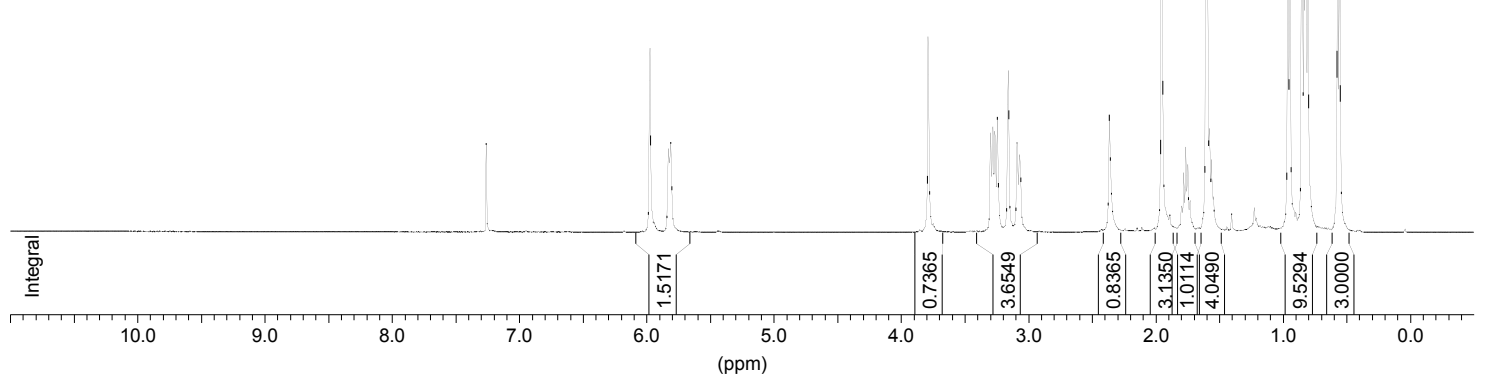




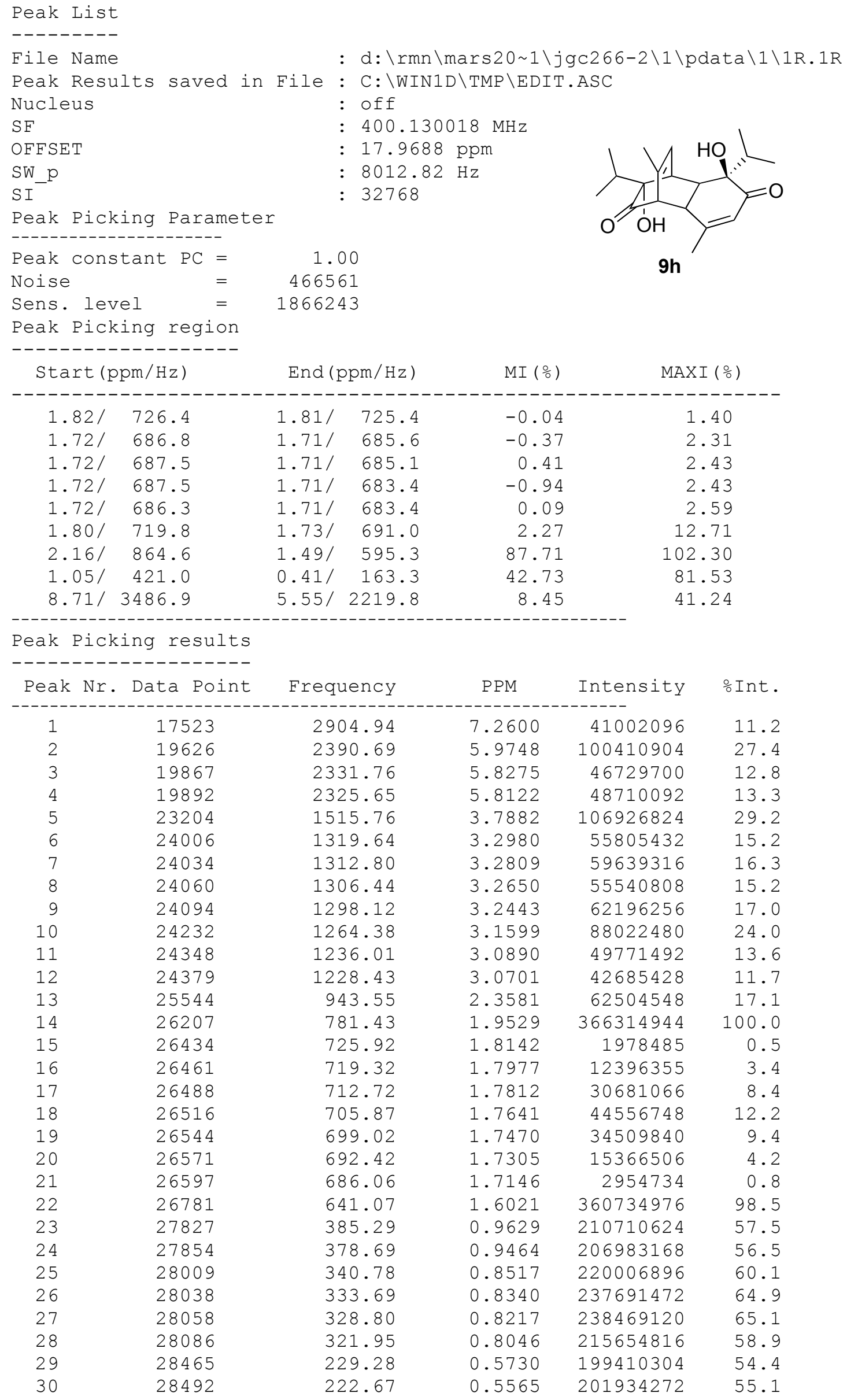




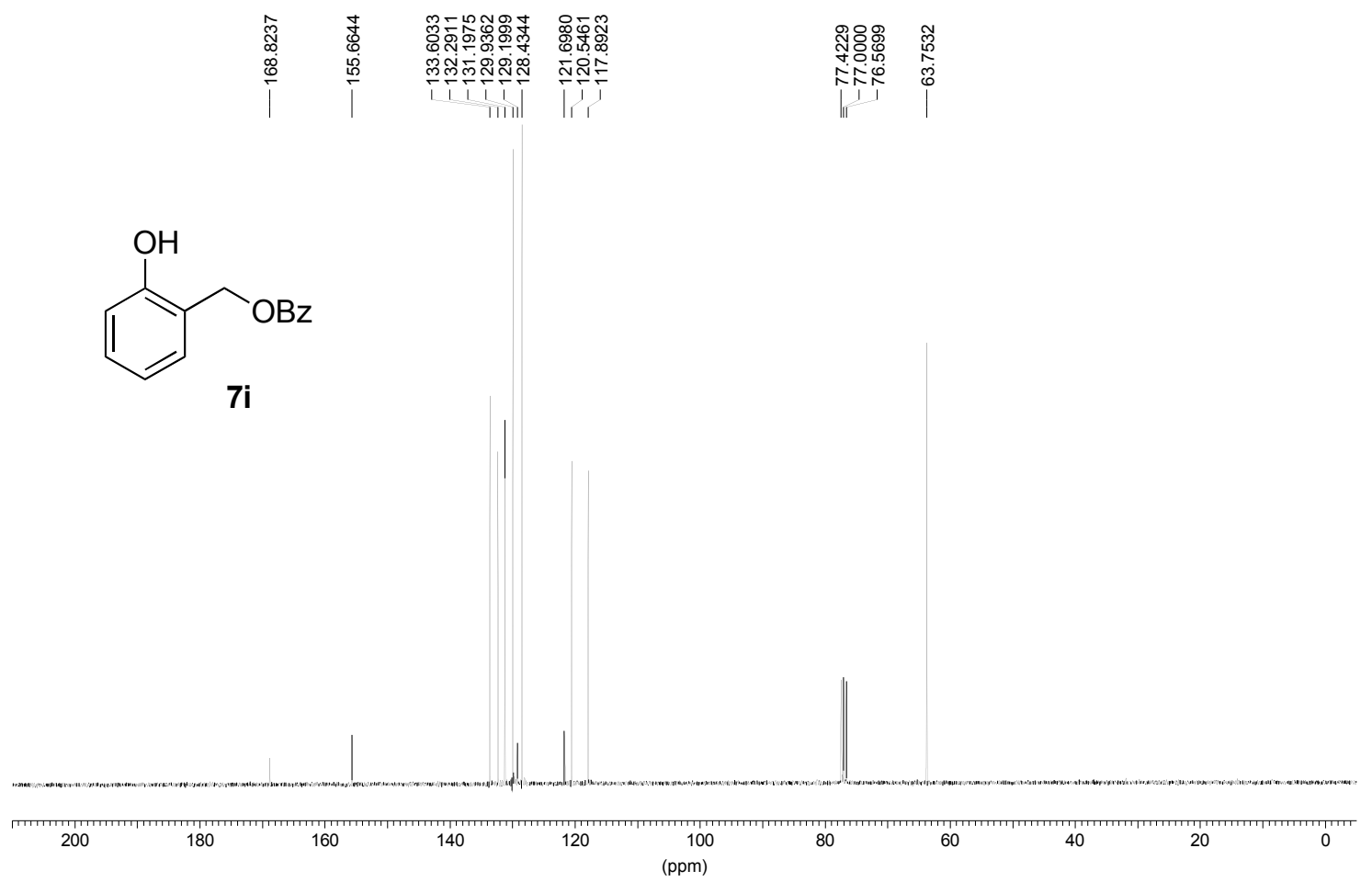

JGE006-2 - DPX300 - 120406 -

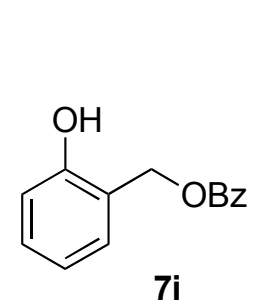

$7 \mathbf{i}$

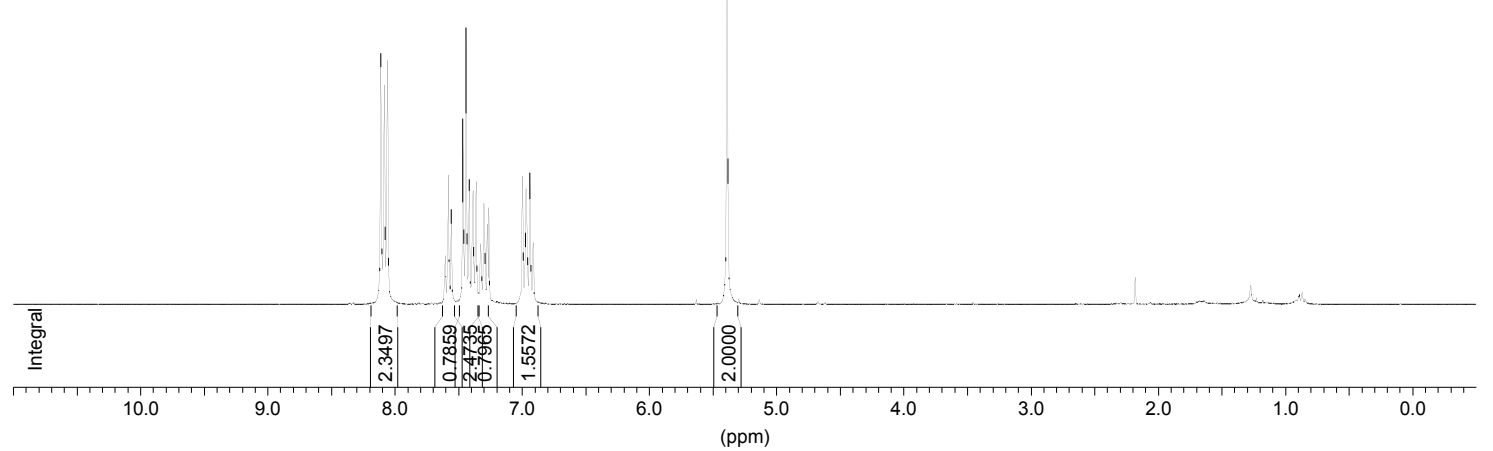


File Name

Peak Results saved in File Nucleus

$\mathrm{SF}$

OFFSET

SW_P

$\mathrm{SI}^{-}$

Peak Picking Parameter

----------------------

Peak constant $\mathrm{PC}=$

Noise

Sens. level=

Peak Picking region

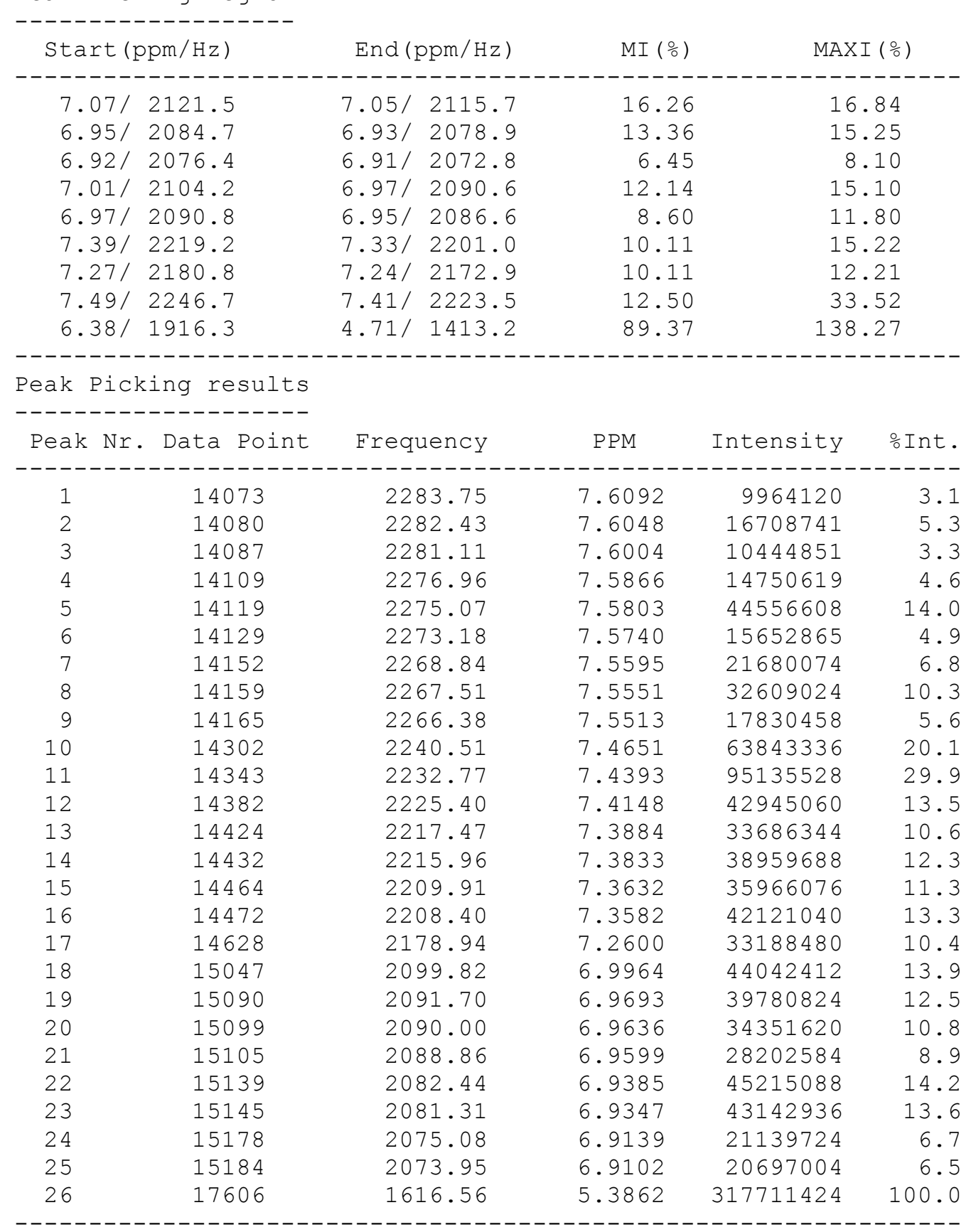

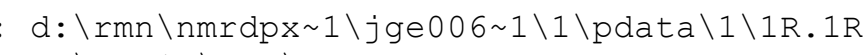

c: \WIN1D TTMP $\backslash E D I T . A S C$

: off

: $300.130006 \mathrm{MHz}$

: $16.4642 \mathrm{ppm}$

: $6188.12 \mathrm{~Hz}$

: 32768

1.00

40369

161478

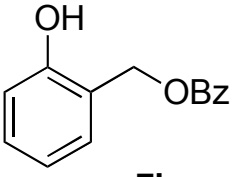

$7 \mathbf{i}$ 
JGE07B-2-R - 300MHz - 230406 -

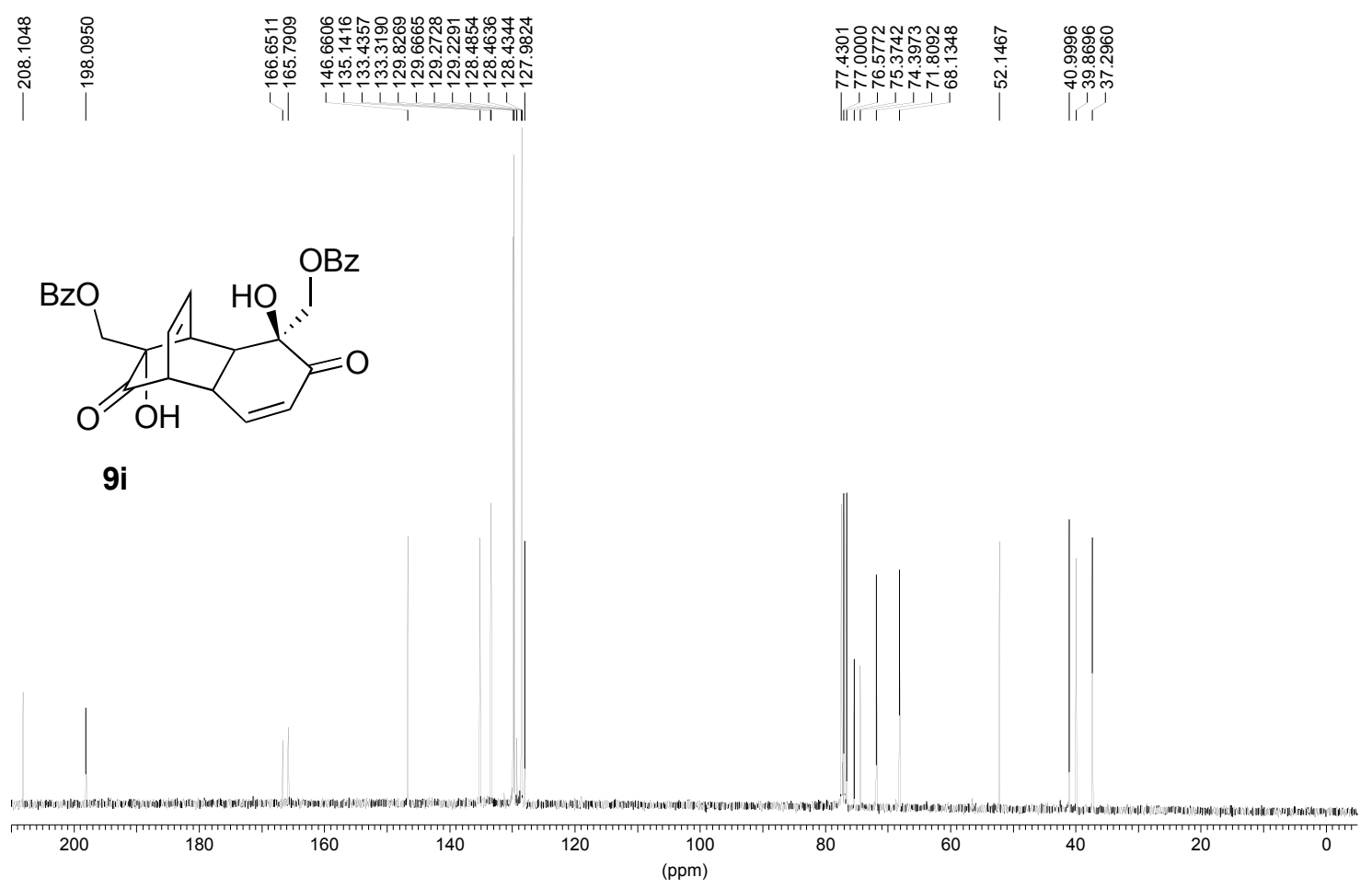

JGE007B-2-R - 300MHz -

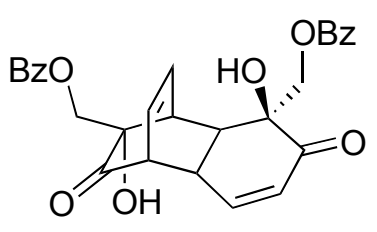

$9 i$

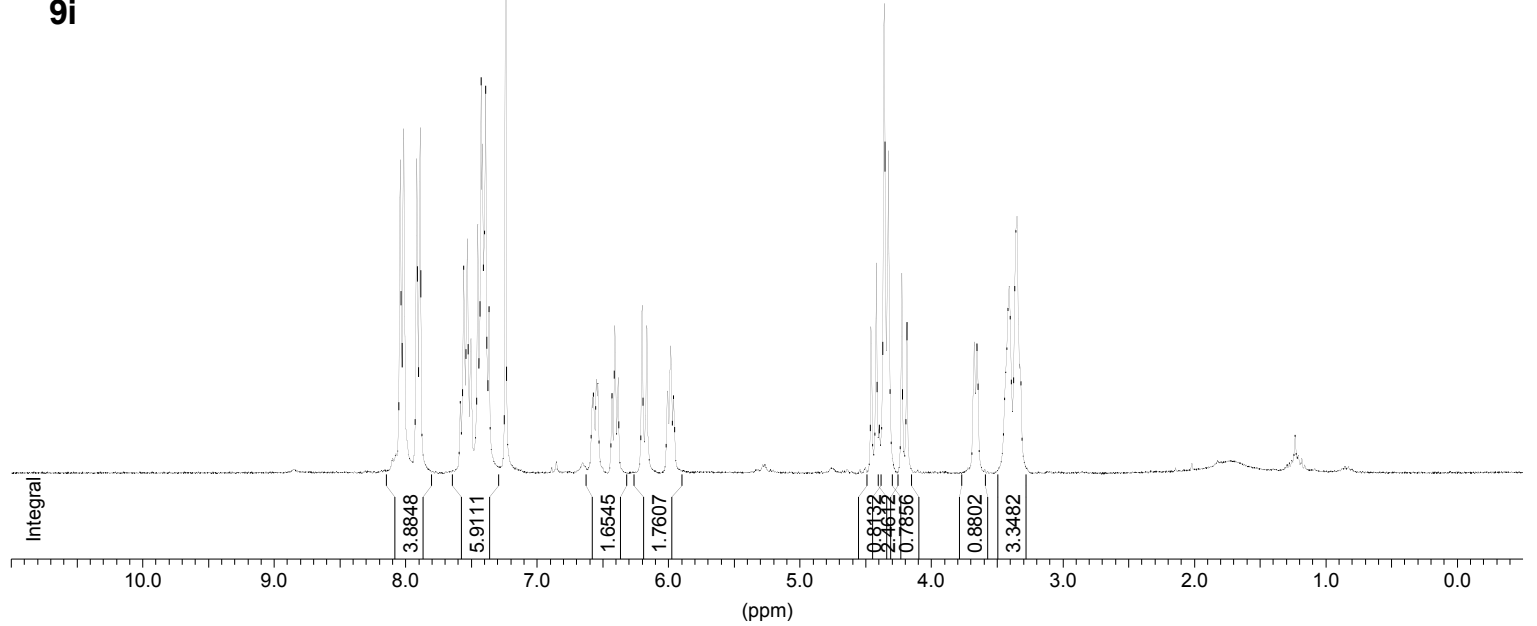




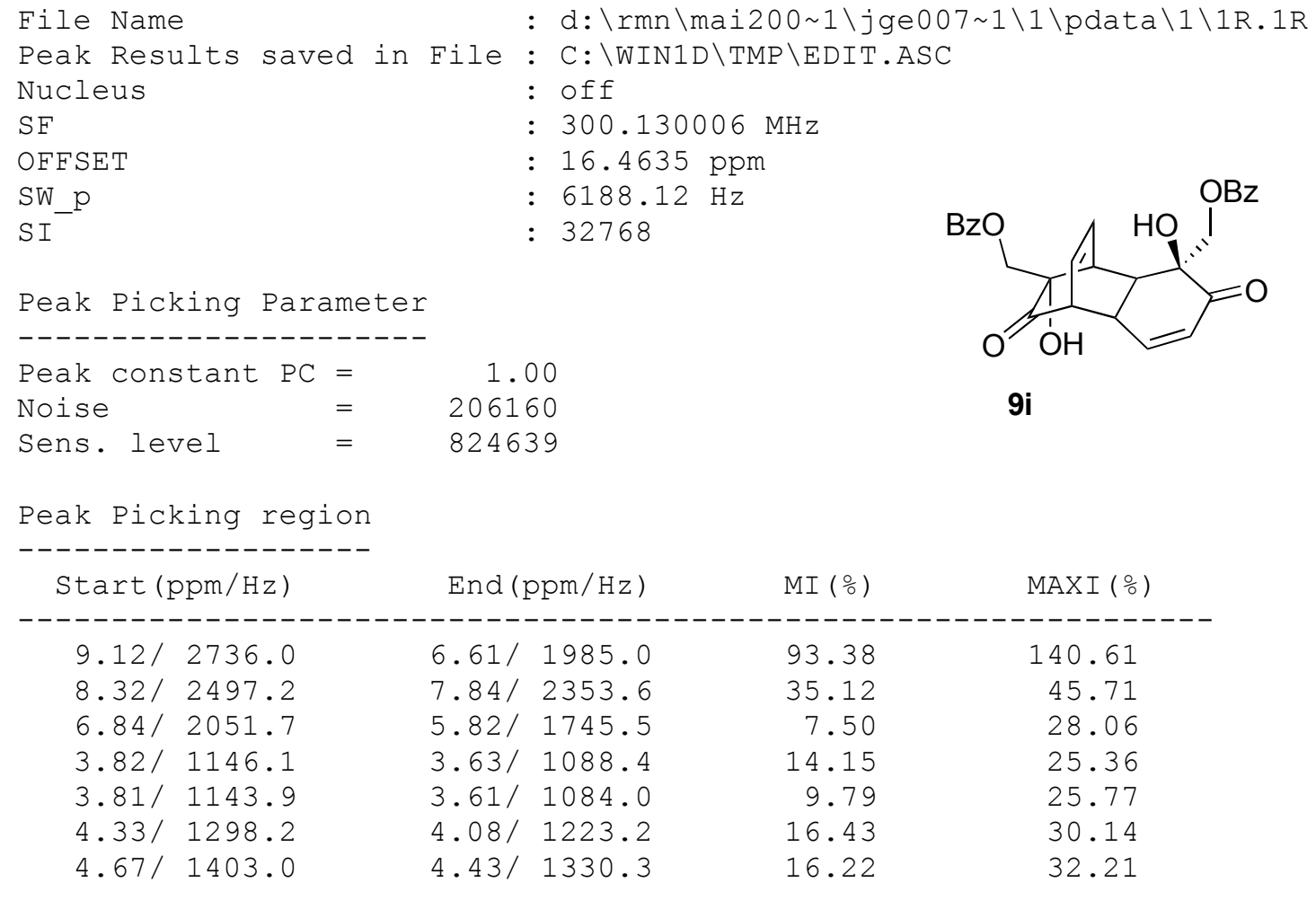

Peak Picking results

\begin{tabular}{|c|c|c|c|c|c|}
\hline Peak & Nr. Data Point & Frequency & PPM & Intensity & $\% \operatorname{Int}$. \\
\hline---- & -------------- & --------- & ---- & --------- & ----- \\
\hline 1 & 13354 & 2419.35 & 8.0610 & 136513184 & 37.5 \\
\hline 2 & 13393 & 2411.98 & 8.0365 & 150018096 & 41.2 \\
\hline 3 & 13552 & 2381.95 & 7.9364 & 137095568 & 37.7 \\
\hline 4 & 13591 & 2374.59 & 7.9119 & 150595264 & 41.4 \\
\hline 5 & 14627 & 2178.94 & 7.2600 & 364004160 & 100.0 \\
\hline 6 & 15671 & 1981.79 & 6.6031 & 34103332 & 9.4 \\
\hline 7 & 15690 & 1978.20 & 6.5911 & 36090004 & 9.9 \\
\hline 8 & 15724 & 1971.78 & 6.5698 & 42200328 & 11.6 \\
\hline 9 & 15743 & 1968.19 & 6.5578 & 40524284 & 11.1 \\
\hline 10 & 15910 & 1936.65 & 6.4527 & 36298164 & 10.0 \\
\hline 11 & 15948 & 1929.48 & 6.4288 & 65546816 & 18.0 \\
\hline 12 & 15986 & 1922.30 & 6.4049 & 42864020 & 11.8 \\
\hline 13 & 16278 & 1867.16 & 6.2212 & 73585480 & 20.2 \\
\hline 14 & 16330 & 1857.34 & 6.1884 & 64611316 & 17.8 \\
\hline 15 & 16585 & 1809.18 & 6.0280 & 35948484 & 9.9 \\
\hline 16 & 16621 & 1802.38 & 6.0053 & 55562332 & 15.3 \\
\hline 17 & 16656 & 1795.77 & 5.9833 & 34015588 & 9.3 \\
\hline 18 & 19045 & 1344.62 & 4.4801 & 66509288 & 18.3 \\
\hline 19 & 19108 & 1332.72 & 4.4405 & 94347032 & 25.9 \\
\hline 20 & 19416 & 1274.56 & 4.2467 & 90293968 & 24.8 \\
\hline 21 & 19479 & 1262.66 & 4.2070 & 68090912 & 18.7 \\
\hline 22 & 20291 & 1109.32 & 3.6961 & 58567752 & 16.1 \\
\hline 23 & 20325 & 1102.90 & 3.6747 & 58082472 & 16.0 \\
\hline
\end{tabular}


AOC97 - 250MHz -

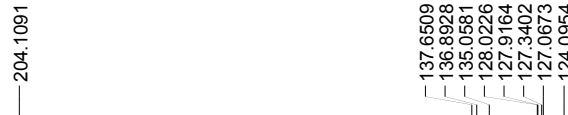

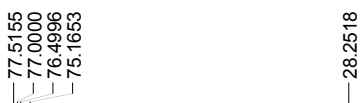<smiles>CC1(O)C=Cc2ccccc2C1=O</smiles>

12

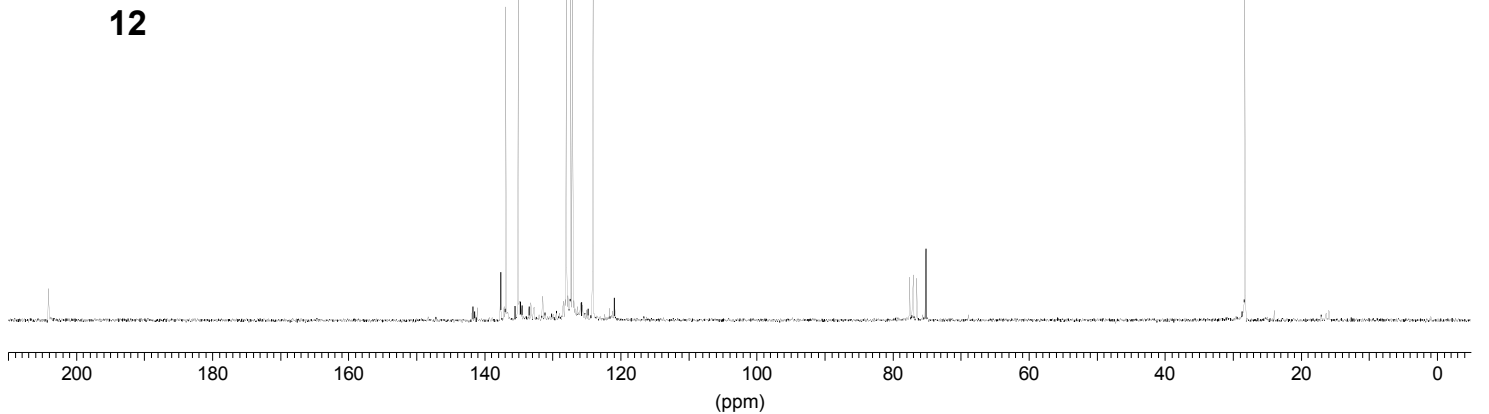

AOC97 - 250MHz -<smiles>CC1(O)C=Cc2ccccc2C1=O</smiles>

12

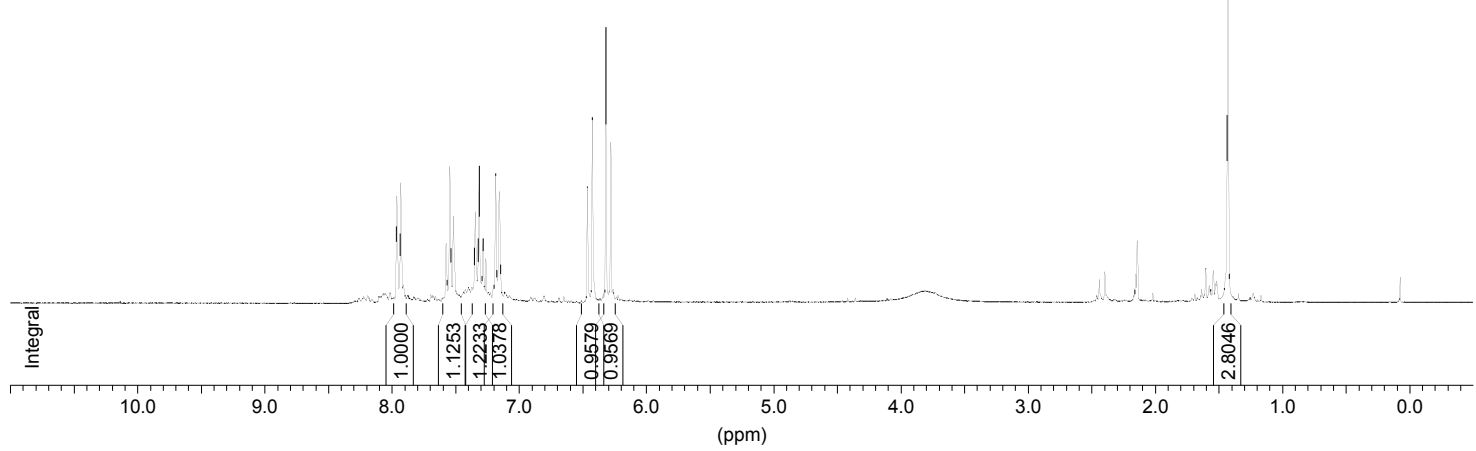




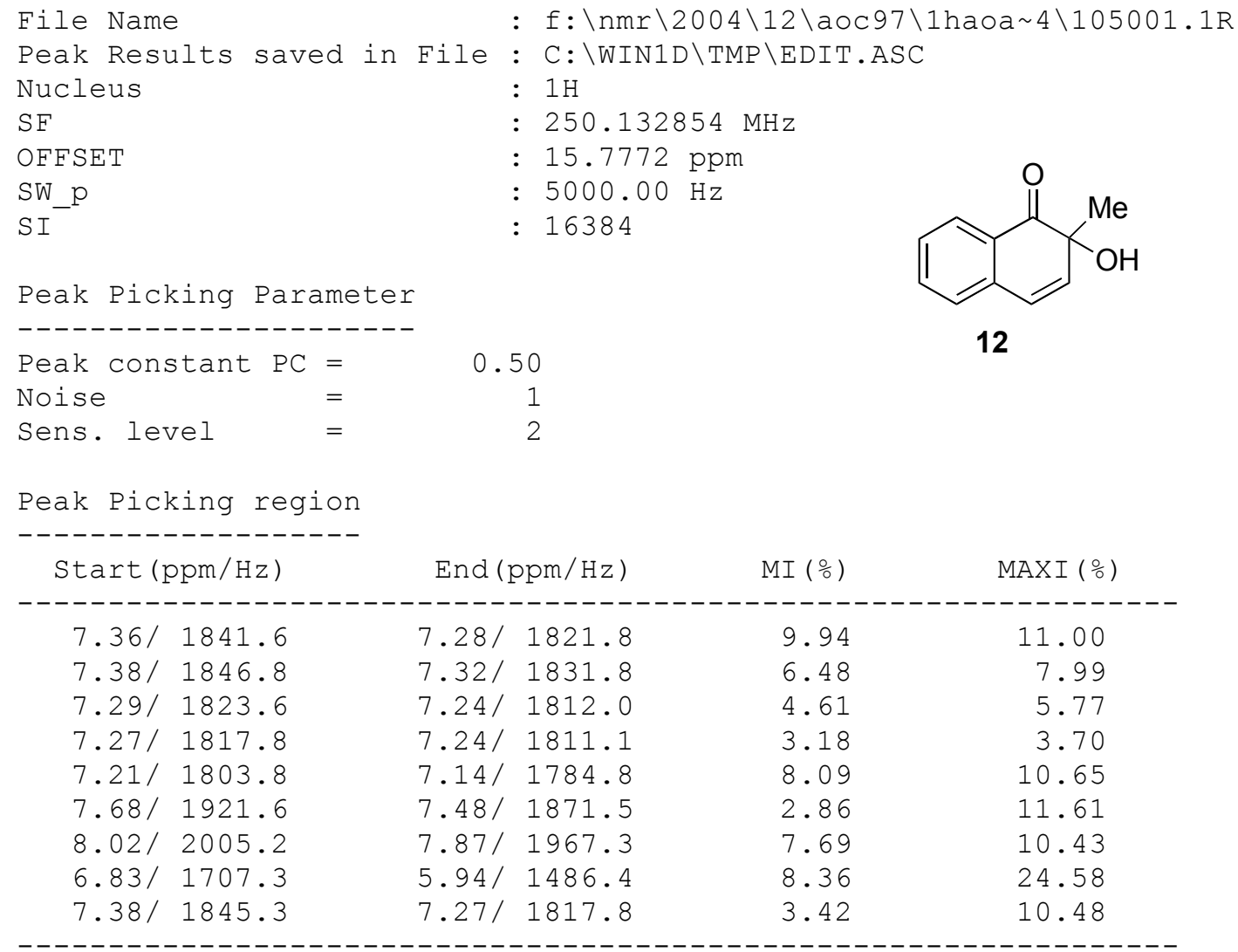

Peak Picking results

\begin{tabular}{|c|c|c|c|c|c|}
\hline Peak & Nr. Data Point & Erequency & PPM & Intensity & Int. \\
\hline--- & -------------- & --------- & ------ & --------- & ----- \\
\hline 1 & 6406 & 1991.44 & 7.9615 & 833 & 7.9 \\
\hline 2 & 6432 & 1983.51 & 7.9298 & 933 & 8.9 \\
\hline 3 & 6720 & 1895.62 & 7.5784 & 311 & 3.0 \\
\hline 4 & 6724 & 1894.39 & 7.5736 & 460 & 4.4 \\
\hline 5 & 6744 & 1888.29 & 7.5492 & 762 & 7.2 \\
\hline 6 & 6749 & 1886.77 & 7.5431 & 1059 & 10.1 \\
\hline 7 & 6769 & 1880.66 & 7.5187 & 547 & 5.2 \\
\hline 8 & 6774 & 1879.14 & 7.5126 & 674 & 6.4 \\
\hline 9 & 6908 & 1838.24 & 7.3491 & 500 & 4.7 \\
\hline 10 & 6912 & 1837.02 & 7.3442 & 706 & 6.7 \\
\hline 11 & 6933 & 1830.61 & 7.3186 & 792 & 7.5 \\
\hline 12 & 6937 & 1829.39 & 7.3137 & 1066 & 10.1 \\
\hline 13 & 6958 & 1822.98 & 7.2881 & 406 & 3.9 \\
\hline 14 & 6962 & 1821.76 & 7.2832 & 497 & 4.7 \\
\hline 15 & 6981 & 1815.96 & 7.2600 & 340 & 3.2 \\
\hline 16 & 7043 & 1797.04 & 7.1844 & 1005 & 9.5 \\
\hline 17 & 7068 & 1789.41 & 7.1539 & 866 & 8.2 \\
\hline 18 & 7634 & 1616.68 & 6.4633 & 903 & 8.6 \\
\hline 19 & 7666 & 1606.92 & 6.4243 & 1442 & 13.7 \\
\hline 20 & 7753 & 1580.37 & 6.3181 & 2147 & 20.4 \\
\hline 21 & 7785 & 1570.60 & 6.2791 & 1249 & 11.9 \\
\hline 22 & 11759 & 357.83 & 1.4306 & 10532 & 100.0 \\
\hline
\end{tabular}


aoc123 - 300MHz - 240105 -

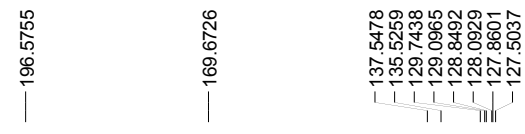

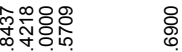

К№<smiles>CC(=O)C1(O)C=Cc2ccccc2C1=O</smiles>

13

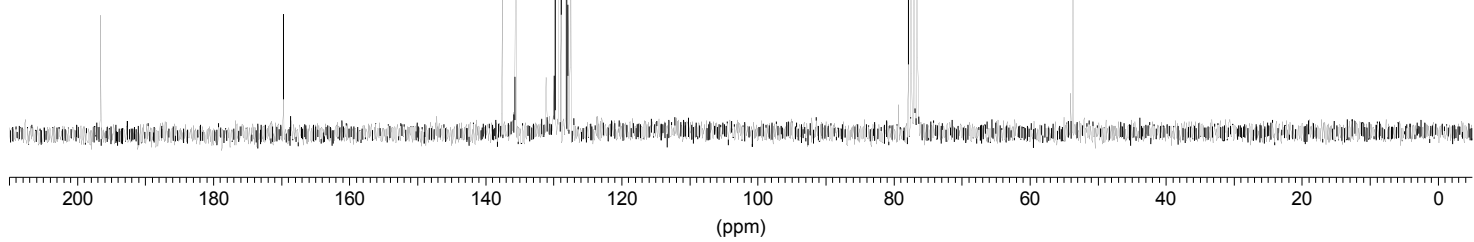

$\mathrm{SQ} 2 / \mathrm{CDCl} 3 / 300 \mathrm{MHz}$<smiles>COC(=O)C1(O)C=Cc2ccccc2C1=O</smiles>

13

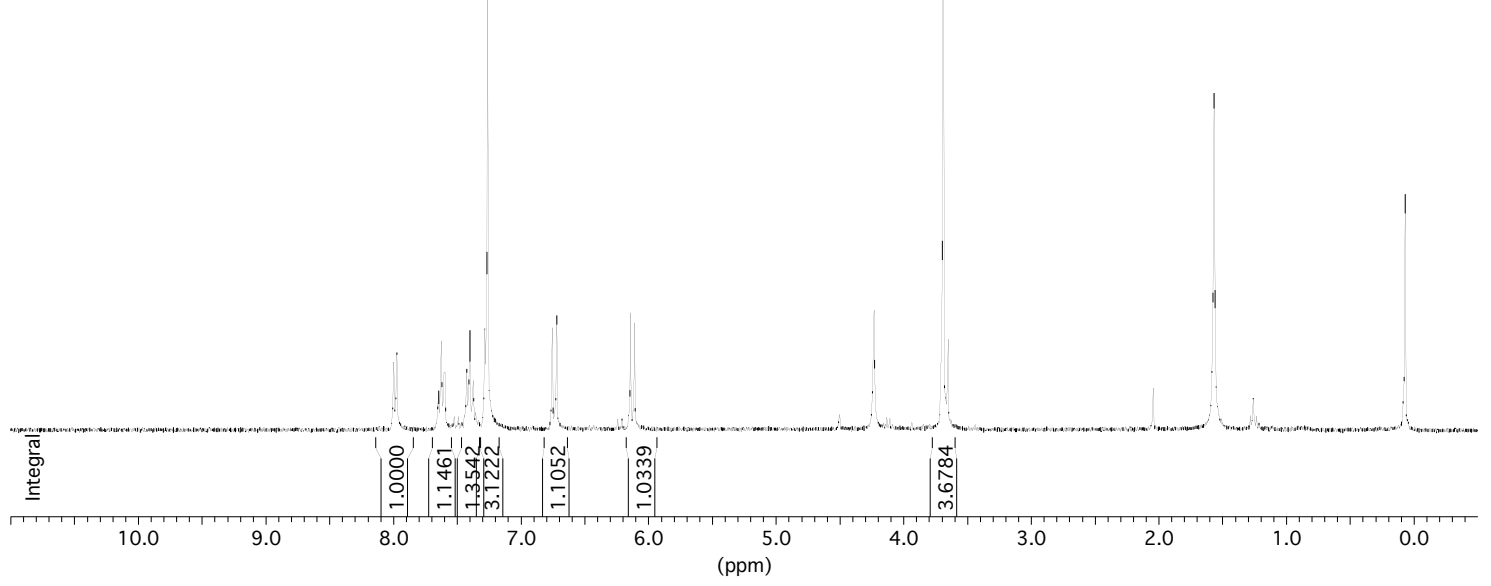




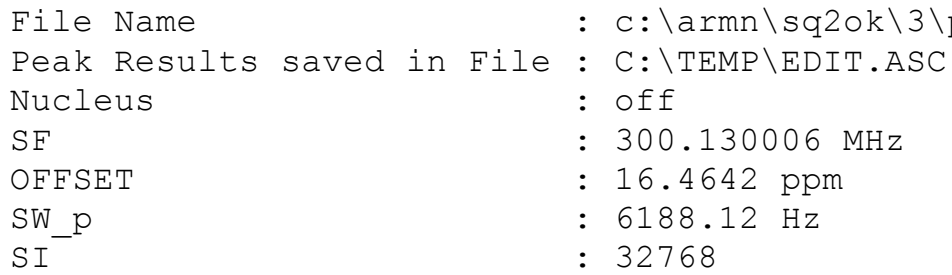

\begin{tabular}{|c|c|c|c|}
\hline Start (ppm/Hz) & End (ppm/Hz) & MI ( $\%)$ & MAXI (응) \\
\hline & 3211 & 6113 & 12701 \\
\hline $6.23 / 1870.0$ & $5.92 / 1776.1$ & $\begin{array}{r}6.13 \\
6.71\end{array}$ & $\begin{array}{r}121.04 \\
25.76\end{array}$ \\
\hline $6.86 / 2057.7$ & $6.64 / 1992.0$ & 8.53 & 27.88 \\
\hline $8.19 / 2458.8$ & $7.35 / 2205.4$ & 3.08 & 29.09 \\
\hline
\end{tabular}

Peak Picking results

\begin{tabular}{|c|c|c|c|c|c|}
\hline Peak & Nr. Data Point & Frequency & PPM & Intensity & \%Int. \\
\hline & & & & & \\
\hline 1 & 13457 & 2400.08 & 7.9968 & 32340990 & 7.6 \\
\hline 2 & 13497 & 2392.53 & 7.9716 & 36739124 & 8.6 \\
\hline 3 & 14015 & 2294.71 & 7.6457 & 18806772 & 4.4 \\
\hline 4 & 14048 & 2288.48 & 7.6249 & 41206592 & 9.7 \\
\hline 5 & 14054 & 2287.34 & 7.6212 & 42302108 & 9.9 \\
\hline 6 & 14087 & 2281.11 & 7.6004 & 27609236 & 6.5 \\
\hline 7 & 14094 & 2279.79 & 7.5960 & 27321884 & 6.4 \\
\hline 8 & 14365 & 2228.61 & 7.4255 & 29093712 & 6.8 \\
\hline 9 & 14406 & 2220.87 & 7.3997 & 47473564 & 11.1 \\
\hline 10 & 14444 & 2213.69 & 7.3758 & 23633632 & 5.5 \\
\hline 11 & 15434 & 2026.73 & 6.7529 & 48508960 & 11.4 \\
\hline 12 & 15486 & 2016.91 & 6.7201 & 54421760 & 12.8 \\
\hline 13 & 16405 & 1843.36 & 6.1419 & 55750972 & 13.1 \\
\hline 14 & 16457 & 1833.54 & 6.1092 & 50983800 & 12.0 \\
\hline 15 & 20300 & 1107.81 & 3.6911 & 426480288 & 100.0 \\
\hline
\end{tabular}




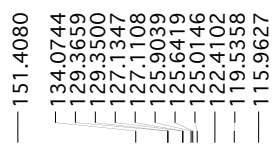<smiles>CC1(Cc2ccc3ccccc3c2O)CO1</smiles>

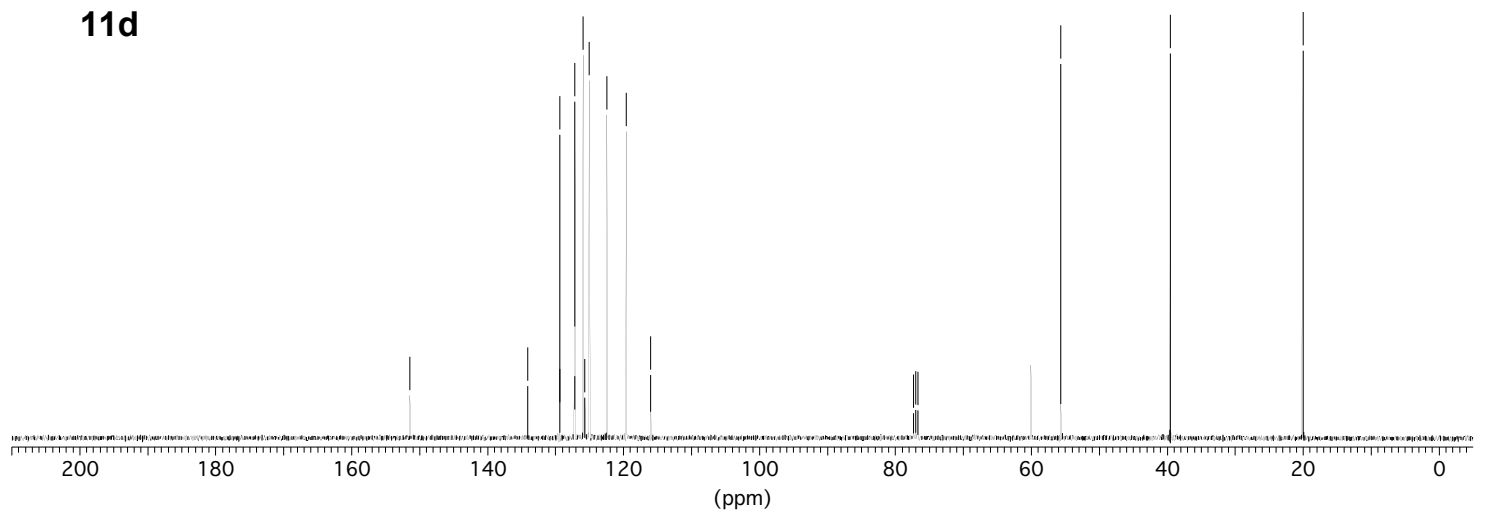<smiles>CC1(Cc2ccc3ccccc3c2O)CO1</smiles>

11d

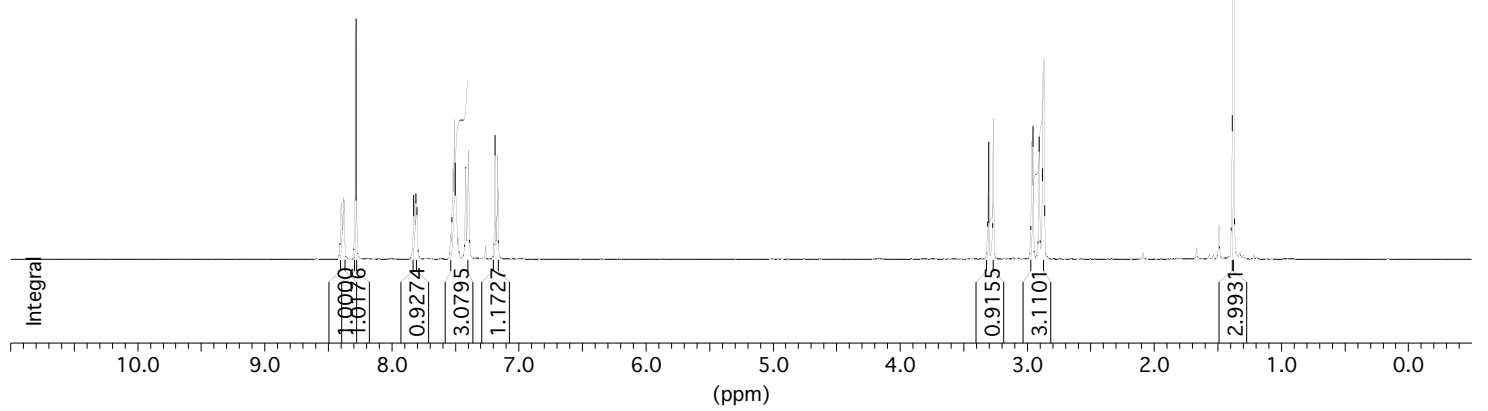




\begin{tabular}{rrrrrr}
\hline Peak Nr. & Data Point & Frequency & PPM & Intensity & \%Int. \\
\hline 1 & 10821 & 3360.74 & 8.3991 & 12709 & 8.0 \\
2 & 10830 & 3358.54 & 8.3936 & 12047 & 7.5 \\
3 & 10858 & 3351.69 & 8.3765 & 13693 & 8.6 \\
4 & 11013 & 3313.79 & 8.2818 & 54516 & 34.1 \\
5 & 11759 & 3131.37 & 7.8259 & 14383 & 9.0 \\
6 & 11787 & 3124.52 & 7.8088 & 14782 & 9.3 \\
7 & 11796 & 3122.32 & 7.8033 & 14420 & 9.0 \\
8 & 12256 & 3009.84 & 7.5221 & 17963 & 11.3 \\
9 & 12263 & 3008.12 & 7.5179 & 20485 & 12.8 \\
10 & 12268 & 3006.90 & 7.5148 & 21801 & 13.7 \\
11 & 12282 & 3003.48 & 7.5063 & 31587 & 19.8 \\
12 & 12295 & 3000.30 & 7.4983 & 21658 & 13.6 \\
13 & 12307 & 2997.36 & 7.4910 & 14383 & 9.0 \\
14 & 12430 & 2967.29 & 7.4158 & 20734 & 13.0 \\
15 & 12464 & 2958.97 & 7.3950 & 24577 & 15.4 \\
16 & 12806 & 2875.34 & 7.1860 & 28019 & 17.5 \\
17 & 12840 & 2867.03 & 7.1652 & 23470 & 14.7 \\
18 & 19159 & 1321.83 & 3.3035 & 26513 & 16.6 \\
19 & 19220 & 1306.92 & 3.2662 & 31827 & 19.9 \\
20 & 19716 & 1185.63 & 2.9631 & 26658 & 16.7 \\
21 & 19732 & 1181.72 & 2.9533 & 30025 & 18.8 \\
22 & 19812 & 1162.15 & 2.9044 & 27643 & 17.3 \\
23 & 19861 & 1150.17 & 2.8745 & 42613 & 26.7 \\
24 & 19876 & 1146.50 & 2.8653 & 45467 & 28.5 \\
25 & 22313 & 550.58 & 1.3760 & 159662 & 100.0 \\
\hline & & & & & \\
& & & & &
\end{tabular}<smiles>CC1(Cc2ccc3ccccc3c2O)CO1</smiles>
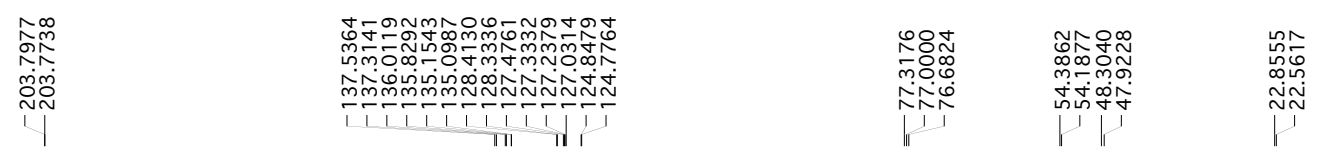<smiles>CC1(CC2(O)C=Cc3ccccc3C2=O)CO1</smiles>

14

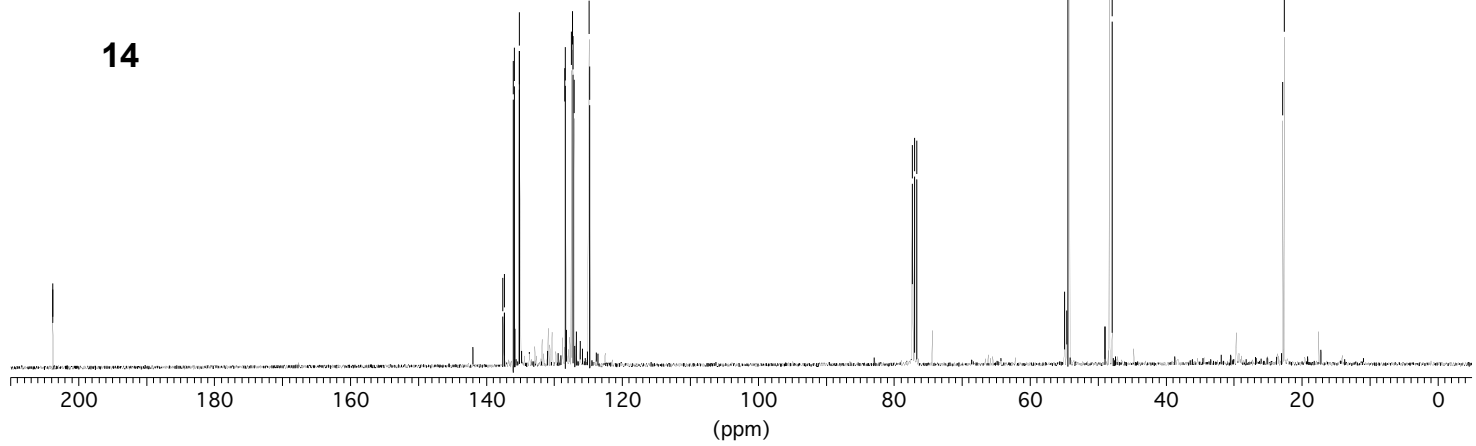


<smiles>CC1(CC2(O)C=Cc3ccccc3C2=O)CO1</smiles>

14

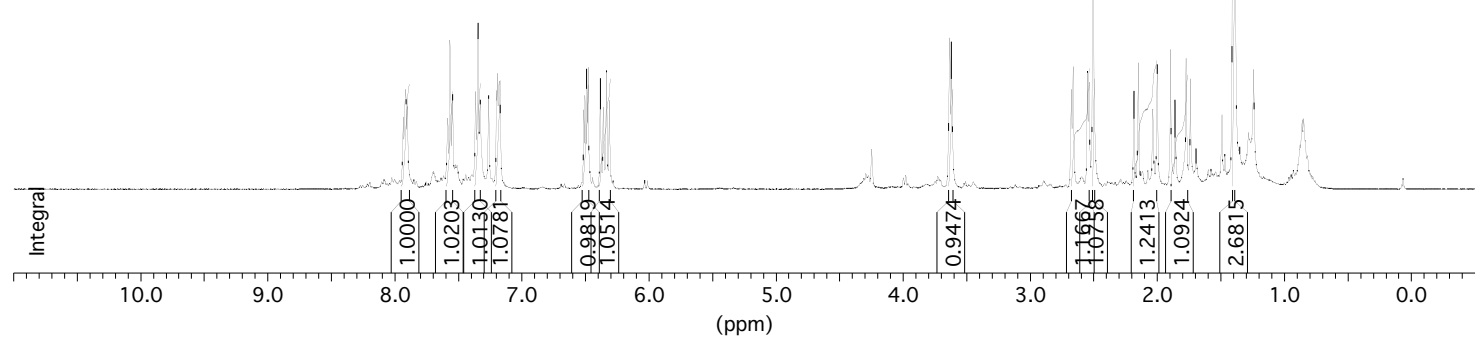

Peak Picking results

Peak Nr. Data Point Frequency PPM Intensity \%Int.

$\begin{array}{ccccccc}1 & 11580 & 3173.93 & 7.9322 & 6102 & 9.9 \\ 2 & 11609 & 3166.84 & 7.9145 & 8558 & 13.9 \\ 3 & 11630 & 3161.70 & 7.9017 & 7054 & 11.4 \\ 4 & 12151 & 3034.30 & 7.5833 & 6006 & 9.7 \\ 5 & 12182 & 3026.72 & 7.5643 & 12895 & 20.9 \\ 6 & 12212 & 3019.39 & 7.5460 & 8135 & 13.2 \\ 7 & 12511 & 2946.27 & 7.3633 & 8336 & 13.5 \\ 8 & 12542 & 2938.69 & 7.3443 & 14425 & 23.4 \\ 9 & 12573 & 2931.11 & 7.3254 & 7640 & 12.4 \\ 10 & 12680 & 2904.94 & 7.2600 & 7996 & 13.0 \\ 11 & 12781 & 2880.25 & 7.1983 & 8466 & 13.7 \\ 12 & 12794 & 2877.07 & 7.1903 & 9976 & 16.2 \\ 13 & 12811 & 2872.91 & 7.1799 & 9273 & 15.0 \\ 14 & 12824 & 2869.73 & 7.1720 & 8982 & 14.6 \\ 15 & 13902 & 2606.13 & 6.5132 & 6722 & 10.9 \\ 16 & 13919 & 2601.97 & 6.5028 & 8024 & 13.0 \\ 17 & 13942 & 2596.34 & 6.4888 & 10365 & 16.8 \\ 18 & 13959 & 2592.19 & 6.4784 & 10513 & 17.0 \\ 19 & 14117 & 2553.55 & 6.3818 & 9548 & 15.5 \\ 20 & 14157 & 2543.77 & 6.3574 & 7008 & 11.4 \\ 21 & 14195 & 2534.48 & 6.3341 & 10214 & 16.6 \\ 22 & 14235 & 2524.70 & 6.3097 & 7580 & 12.3 \\ 23 & 18613 & 1454.14 & 3.6342 & 13124 & 21.3\end{array}$




$\begin{array}{cccccc}24 & 18639 & 1447.78 & 3.6183 & 12768 & 20.7 \\ 25 & 20184 & 1069.98 & 2.6741 & 8554 & 13.9 \\ 26 & 20203 & 1065.33 & 2.6625 & 10562 & 17.1 \\ 27 & 20390 & 1019.60 & 2.5482 & 10186 & 16.5 \\ 28 & 20409 & 1014.96 & 2.5366 & 9194 & 14.9 \\ 29 & 20464 & 1001.51 & 2.5030 & 37163 & 60.2 \\ 30 & 20986 & 873.86 & 2.1839 & 8428 & 13.7 \\ 31 & 21045 & 859.44 & 2.1479 & 10895 & 17.7 \\ 32 & 21229 & 814.44 & 2.0354 & 6802 & 11.0 \\ 33 & 21287 & 800.26 & 2.0000 & 10744 & 17.4 \\ 34 & 21461 & 757.71 & 1.8937 & 12085 & 19.6 \\ 35 & 21519 & 743.53 & 1.8582 & 7634 & 12.4 \\ 36 & 21659 & 709.29 & 1.7727 & 11303 & 18.3 \\ 37 & 21717 & 695.11 & 1.7372 & 9463 & 15.3 \\ 38 & 22262 & 561.84 & 1.4041 & 61549 & 99.8 \\ 39 & 22285 & 556.22 & 1.3901 & 61697 & 100.0\end{array}$




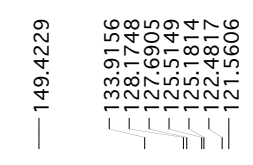<smiles>FC(F)(F)Oc1c(CC2CO2)ccc2ccccc12</smiles>

17

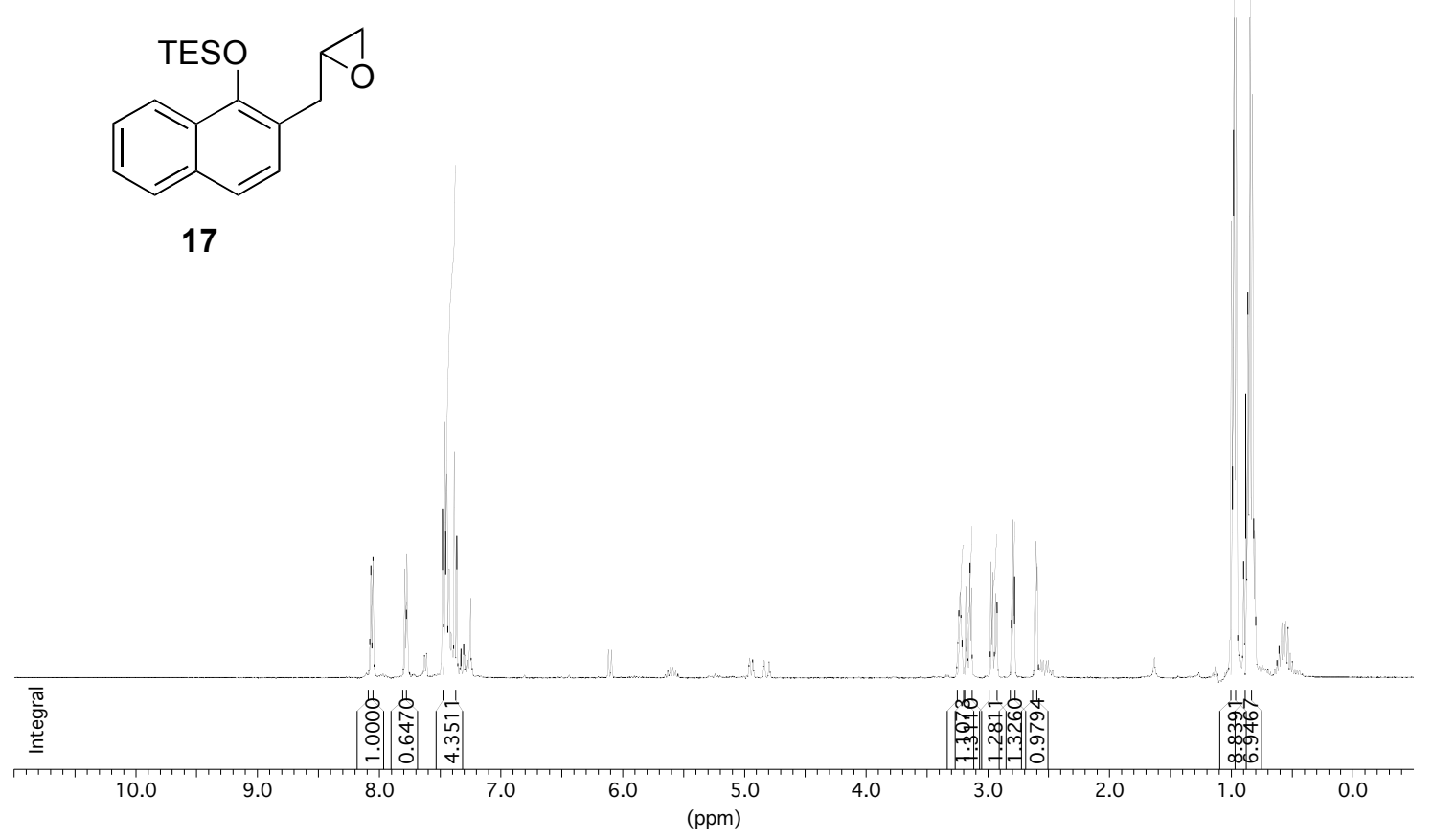


Peak Picking results

$\begin{array}{llccccc}\text { Peak Nr. Data Point } & \text { Frequency } & \text { PPM } & \text { Intensity } & \text { \%Int. } \\ -1 & 11339 & 3233.59 & 8.0814 & 3486 & 7.8 \\ 2 & 11371 & 3225.77 & 8.0618 & 3747 & 8.4 \\ 3 & 11797 & 3121.60 & 7.8015 & 3375 & 7.6 \\ 4 & 11826 & 3114.51 & 7.7837 & 3868 & 8.7 \\ 5 & 12307 & 2996.89 & 7.4898 & 5299 & 11.9 \\ 6 & 12340 & 2988.82 & 7.4696 & 8063 & 18.0 \\ 7 & 12359 & 2984.17 & 7.4580 & 6404 & 14.3 \\ 8 & 12366 & 2982.46 & 7.4537 & 5880 & 13.2 \\ 9 & 12390 & 2976.59 & 7.4391 & 3373 & 7.5 \\ 10 & 12395 & 2975.37 & 7.4360 & 3363 & 7.5 \\ 11 & 12464 & 2958.50 & 7.3938 & 7112 & 15.9 \\ 12 & 12498 & 2950.18 & 7.3731 & 4434 & 9.9 \\ 13 & 12683 & 2904.94 & 7.2600 & 2453 & 5.5 \\ 14 & 19258 & 1297.15 & 3.2418 & 2634 & 5.9 \\ 15 & 19337 & 1277.83 & 3.1935 & 2807 & 6.3 \\ 16 & 19358 & 1272.69 & 3.1807 & 1627 & 3.6 \\ 17 & 19395 & 1263.65 & 3.1581 & 3571 & 8.0 \\ 18 & 19417 & 1258.27 & 3.1446 & 2776 & 6.2 \\ 19 & 19677 & 1194.69 & 2.9857 & 3605 & 8.1 \\ 20 & 19699 & 1189.31 & 2.9723 & 3276 & 7.3 \\ 21 & 19735 & 1180.51 & 2.9503 & 2595 & 5.8 \\ 22 & 19758 & 1174.88 & 2.9362 & 2312 & 5.2 \\ 23 & 19957 & 1126.22 & 2.8146 & 3033 & 6.8 \\ 24 & 19974 & 1122.06 & 2.8042 & 4945 & 11.1 \\ 25 & 19993 & 1117.42 & 2.7926 & 3140 & 7.0\end{array}$



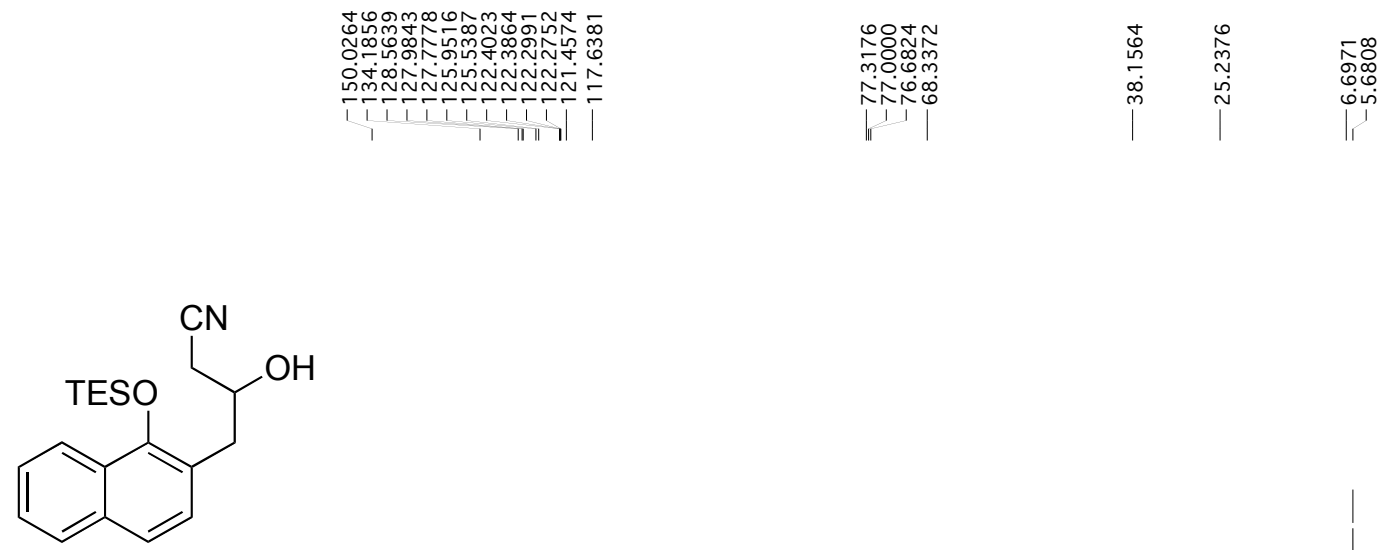

$18 a$
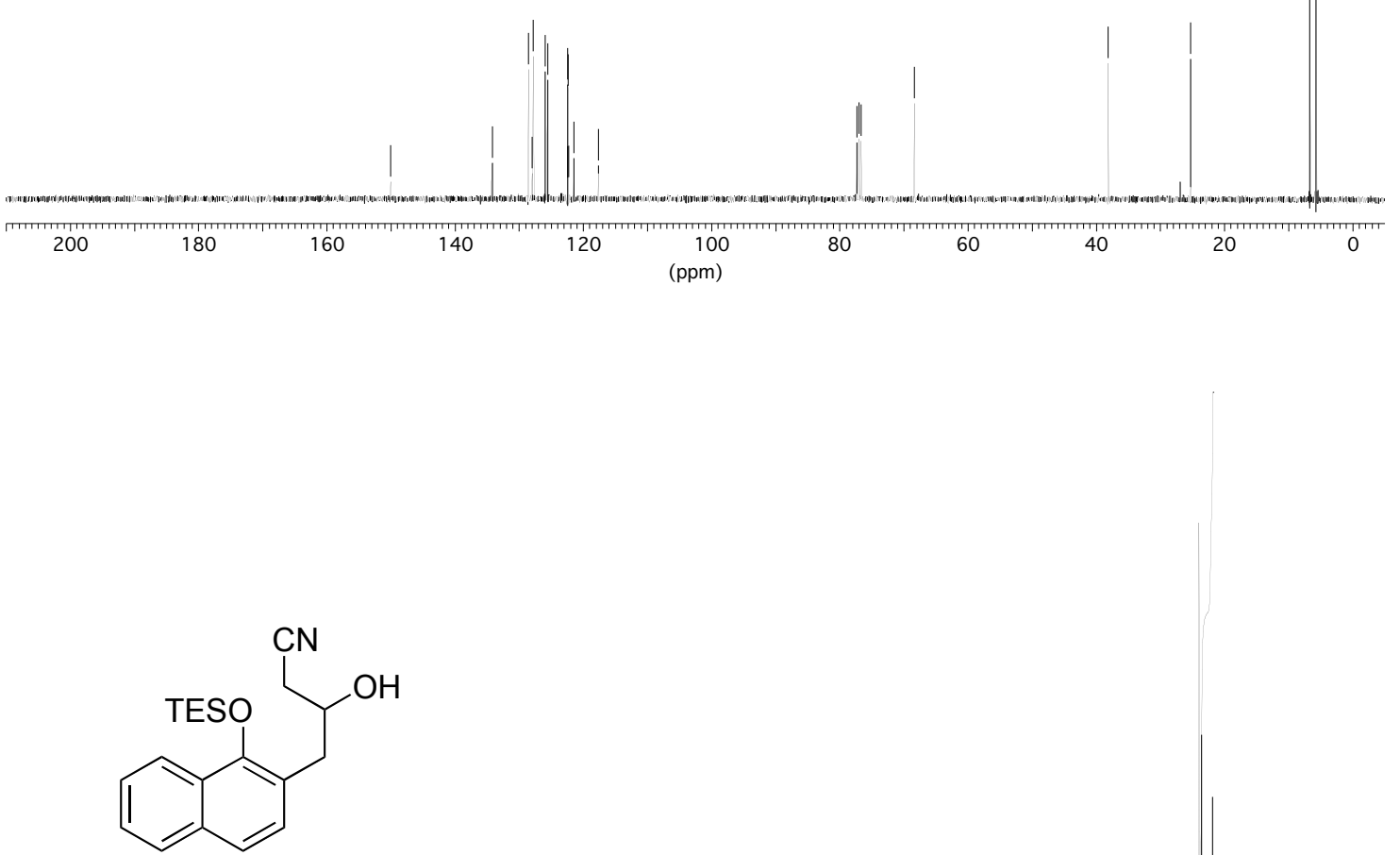

$18 a$

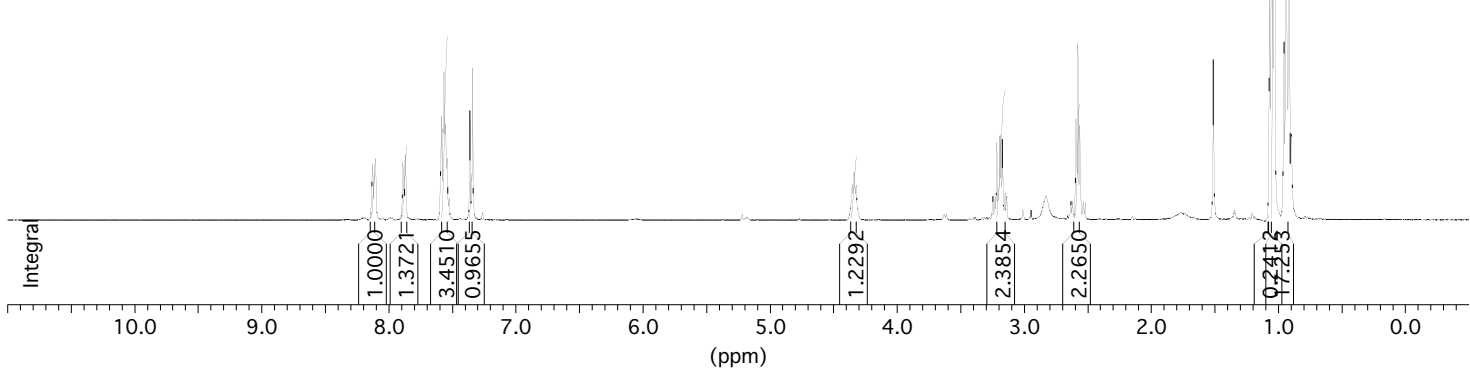


Peak Picking results

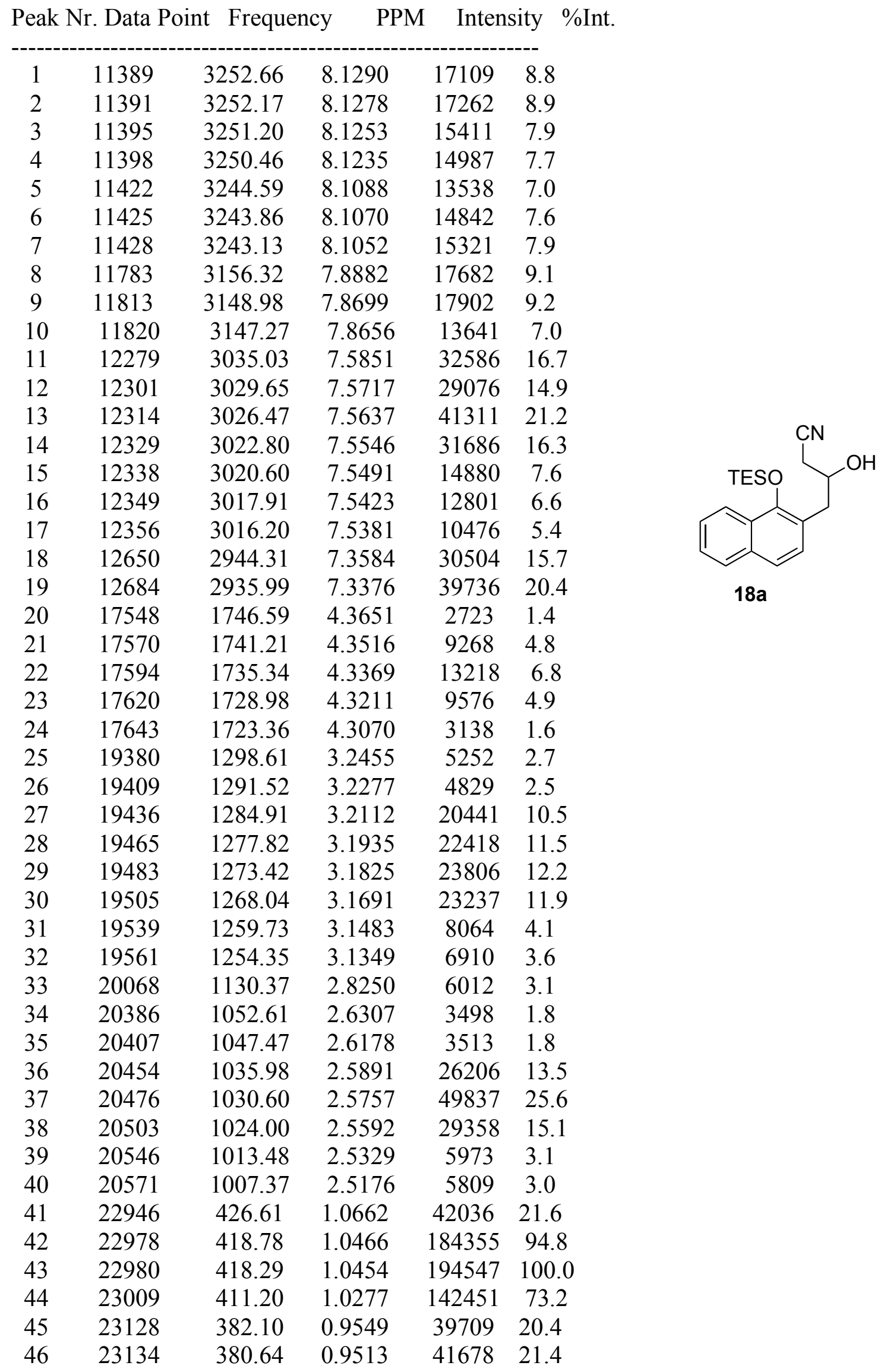


$\begin{array}{llllll}47 & 23160 & 374.28 & 0.9354 & 109850 & 56.5\end{array}$

$\begin{array}{llllll}48 & 23194 & 365.96 & 0.9146 & 93039 & 47.8\end{array}$

$\begin{array}{llllll}49 & 23223 & 358.87 & 0.8969 & 36967 & 19.0\end{array}$

$\begin{array}{llllll}50 & 23231 & 356.92 & 0.8920 & 36679 & 18.9\end{array}$

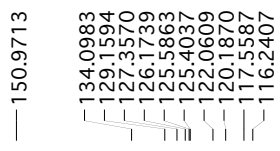<smiles>N#CCC(O)Cc1ccc2ccccc2c1O</smiles>

$18 \mathrm{~b}$<smiles>N#CCC(O)Cc1ccc2ccccc2c1O</smiles>

18b

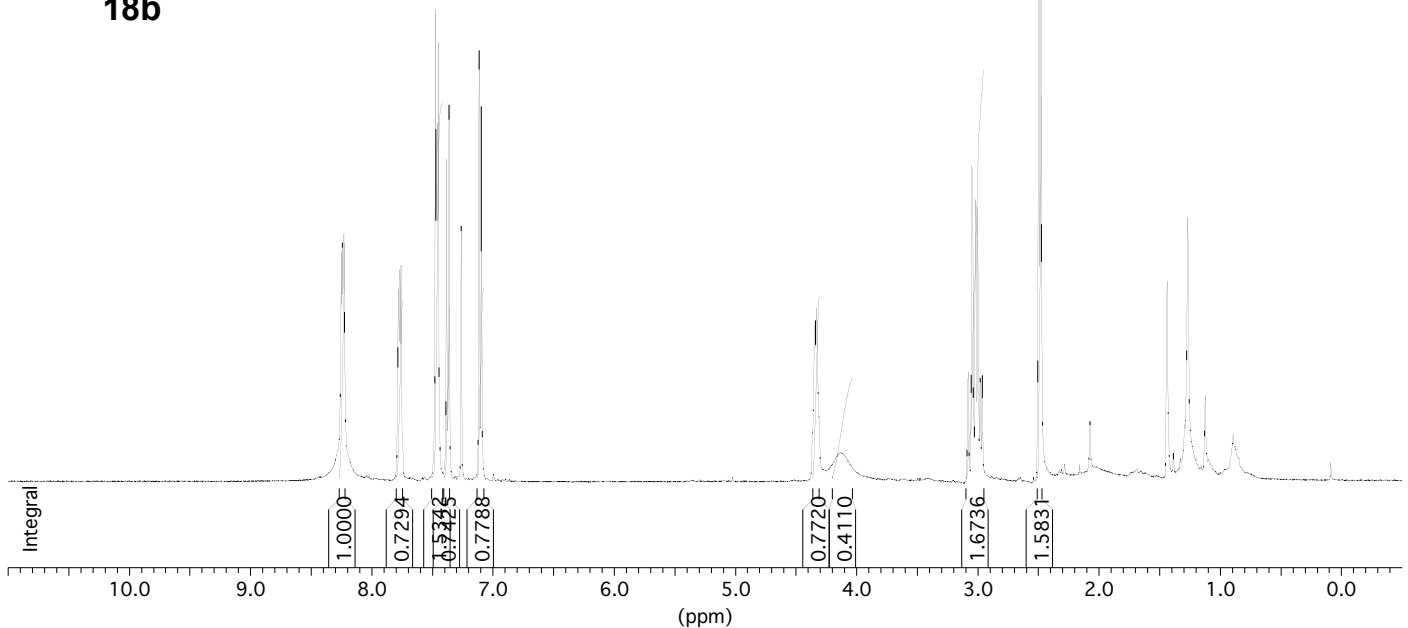


Peak Nr. Data Point Frequency PPM Intensity \%Int.

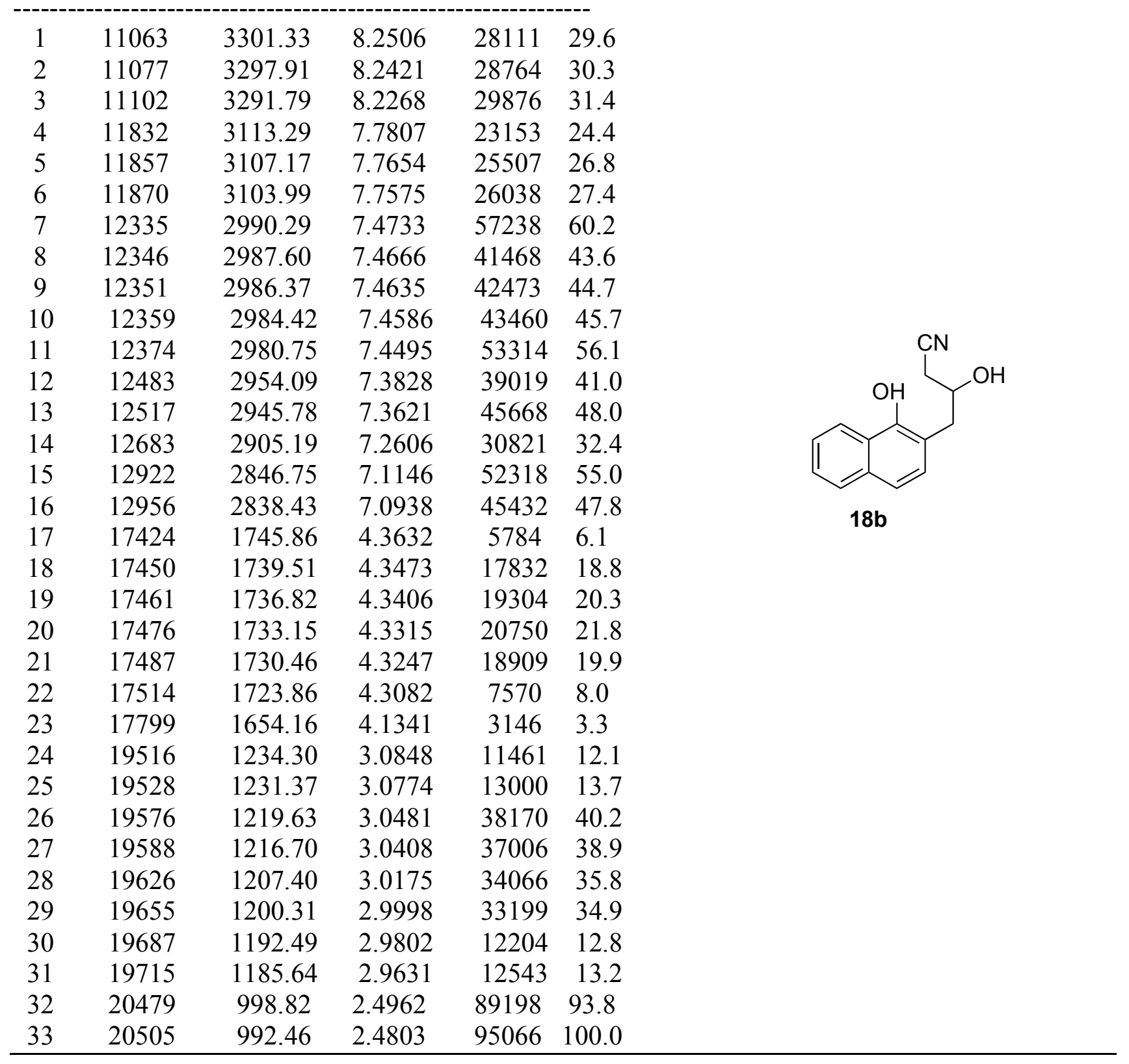



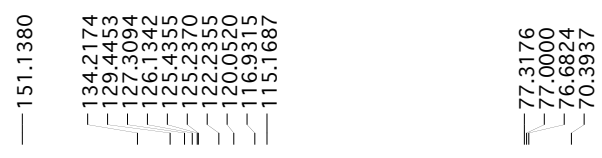

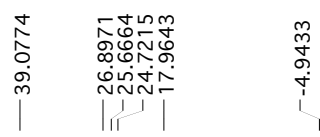<smiles>CC(C)[O+]SC(CC#N)Cc1ccc2ccccc2c1O</smiles>

$11 \mathrm{e}$<smiles>CC(C)(C)OC(CC#N)Cc1ccc2ccccc2c1O</smiles>

$11 \mathrm{e}$

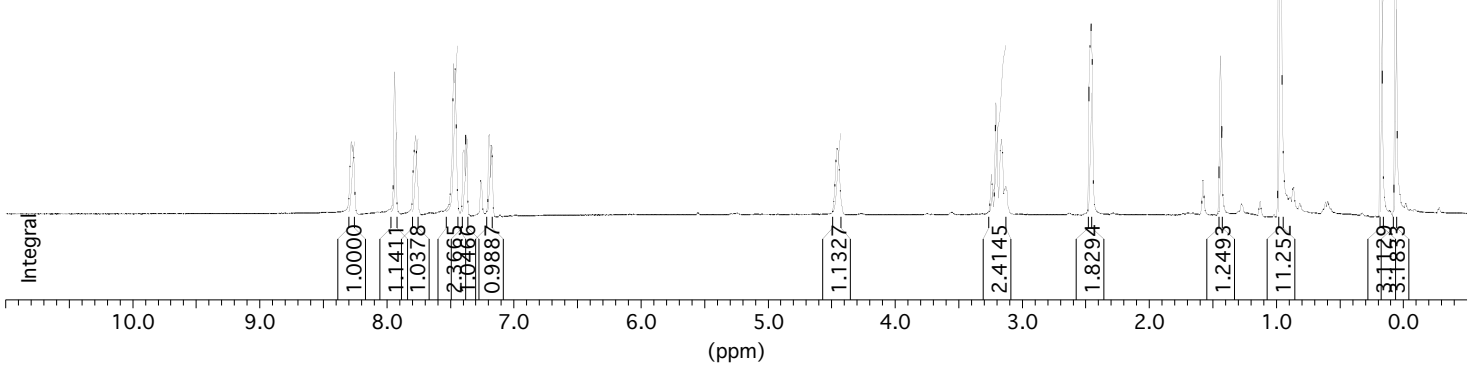


Peak Picking results

\begin{tabular}{cccccc} 
Peak Nr. Data Point & Frequency & PPM & Intensity & \%Int. \\
\hline 1 & 10995 & 3312.58 & 8.2788 & 12303 & 5.1 \\
2 & 11015 & 3307.69 & 8.2665 & 11751 & 4.8 \\
3 & 11548 & 3177.35 & 7.9408 & 24449 & 10.0 \\
4 & 11557 & 3175.15 & 7.9353 & 23476 & 9.6 \\
5 & 11811 & 3113.04 & 7.7801 & 12034 & 4.9 \\
6 & 11830 & 3108.39 & 7.7685 & 13352 & 5.5 \\
7 & 12312 & 2990.53 & 7.4739 & 25914 & 10.6 \\
8 & 12331 & 2985.88 & 7.4623 & 25058 & 10.3 \\
9 & 12446 & 2957.76 & 7.3920 & 10816 & 4.4 \\
10 & 12470 & 2951.89 & 7.3773 & 13446 & 5.5 \\
12 & 12662 & 2904.94 & 7.2600 & 5537 & 2.3 \\
13 & 12674 & 2902.01 & 7.2527 & 5384 & 2.2 \\
14 & 12763 & 2880.25 & 7.1983 & 13195 & 5.4 \\
15 & 12796 & 2877.56 & 7.1916 & 13566 & 5.6 \\
16 & 17253 & 1782.30 & 4.4543 & 11111 & 4.6 \\
17 & 17264 & 1779.61 & 4.4476 & 11103 & 4.6 \\
18 & 19236 & 1297.39 & 3.2424 & 6572 & 2.7 \\
19 & 19298 & 1282.23 & 3.2045 & 19085 & 7.8 \\
20 & 19365 & 1265.85 & 3.1636 & 12721 & 5.2 \\
21 & 19404 & 1256.31 & 3.1398 & 4101 & 1.7 \\
22 & 19427 & 1250.69 & 3.1257 & 4522 & 1.9 \\
23 & 20513 & 985.12 & 2.4620 & 31769 & 13.0 \\
24 & 20519 & 983.66 & 2.4583 & 32861 & 13.5 \\
25 & 22191 & 574.80 & 1.4365 & 27287 & 11.2 \\
26 & 22953 & 388.47 & 0.9709 & 243452 & 100.0 \\
27 & 24257 & 69.60 & 0.1739 & 75573 & 31.0 \\
28 & 24446 & 23.38 & 0.0584 & 76037 & 31.2 \\
\hline
\end{tabular}




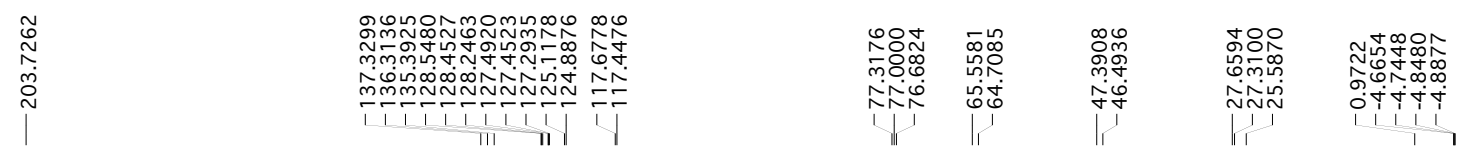<smiles>CCC(C)(CC1(O)C=Cc2ccccc2C1=O)C([Se])(CC#N)[Se]O</smiles>

15

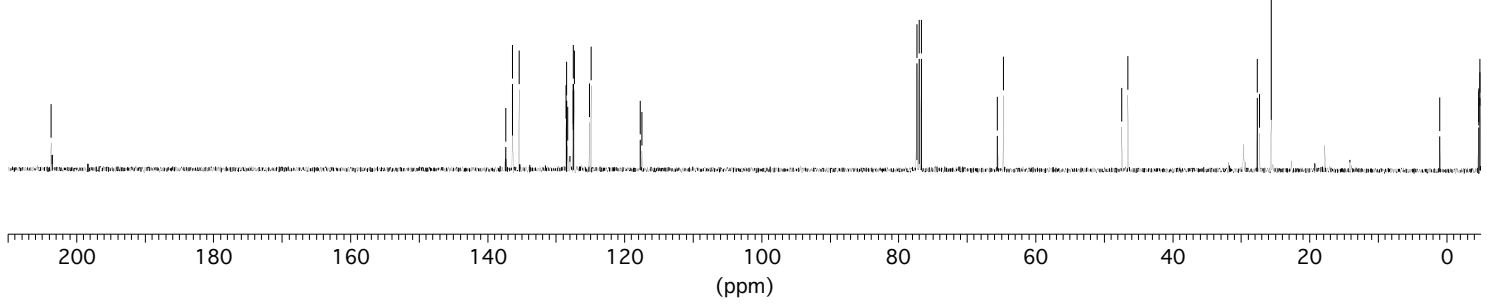<smiles>CCC(C)(CC1(O)C=Cc2ccccc2C1=O)C([Se])[Se-]</smiles>

15

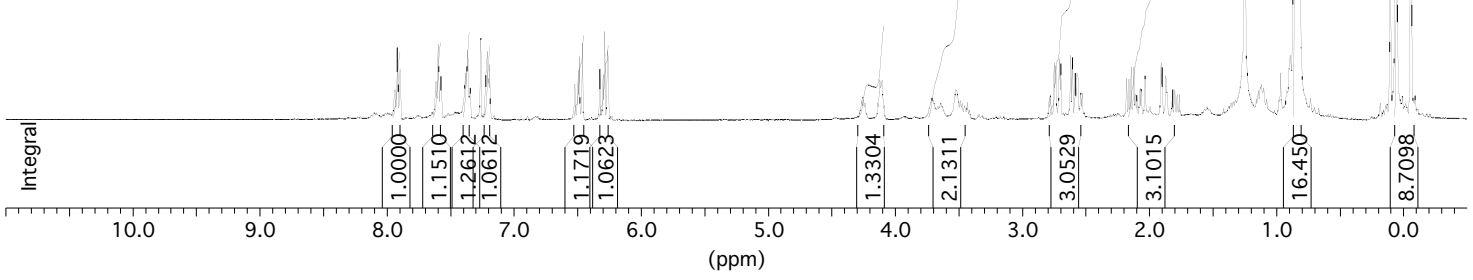


Peak Nr. Data Point Frequency PPM Intensity \%Int.

$\begin{array}{cccccc}------------------------------- \\ 1 & 11576 & 3175.64 & 7.9365 & 4085 & 1.7 \\ 2 & 11610 & 3167.33 & 7.9157 & 10380 & 4.3 \\ 3 & 11643 & 3159.26 & 7.8956 & 7137 & 2.9 \\ 4 & 12106 & 3046.04 & 7.6126 & 5325 & 2.2 \\ 5 & 12111 & 3044.82 & 7.6096 & 4403 & 1.8 \\ 6 & 12136 & 3038.70 & 7.5943 & 10816 & 4.5 \\ 7 & 12142 & 3037.24 & 7.5906 & 7984 & 3.3 \\ 8 & 12167 & 3031.12 & 7.5753 & 6099 & 2.5 \\ 9 & 12173 & 3029.66 & 7.5717 & 4201 & 1.7 \\ 10 & 12473 & 2956.30 & 7.3883 & 4791 & 2.0 \\ 11 & 12484 & 2953.61 & 7.3816 & 6581 & 2.7 \\ 12 & 12487 & 2952.87 & 7.3798 & 6569 & 2.7 \\ 13 & 12504 & 2948.72 & 7.3694 & 7782 & 3.2 \\ 14 & 12514 & 2946.27 & 7.3633 & 9969 & 4.1 \\ 15 & 12531 & 2942.11 & 7.3529 & 3604 & 1.5 \\ 16 & 12535 & 2941.13 & 7.3504 & 3567 & 1.5 \\ 17 & 12545 & 2938.69 & 7.3443 & 4363 & 1.8 \\ 18 & 12683 & 2904.94 & 7.2600 & 11752 & 4.9 \\ 19 & 12750 & 2888.56 & 7.2191 & 6230 & 2.6 \\ 20 & 12765 & 2884.89 & 7.2099 & 9746 & 4.0 \\ 21 & 12779 & 2881.47 & 7.2013 & 5788 & 2.4 \\ 22 & 12795 & 2877.56 & 7.1916 & 7836 & 3.2 \\ 23 & 13893 & 2609.06 & 6.5205 & 4894 & 2.0 \\ 24 & 13934 & 2599.03 & 6.4955 & 6961 & 2.9 \\ 25 & 13953 & 2594.39 & 6.4839 & 9028 & 3.7 \\ 26 & 13993 & 2584.61 & 6.4594 & 11022 & 4.6 \\ 27 & 14217 & 2529.83 & 6.3225 & 7151 & 3.0 \\ 28 & 14257 & 2520.05 & 6.2981 & 6352 & 2.6 \\ 29 & 14278 & 2514.92 & 6.2852 & 12760 & 5.3 \\ 30 & 14318 & 2505.13 & 6.2608 & 9869 & 4.1 \\ 31 & 17563 & 1711.63 & 4.2777 & 962 & 0.4 \\ 32 & 17584 & 1706.49 & 4.2648 & 2463 & 1.0 \\ 33 & 17604 & 1701.60 & 4.2526 & 2930 & 1.2 \\ 34 & 17632 & 1694.76 & 4.2355 & 2096 & 0.9 \\ 35 & 17795 & 1654.90 & 4.1359 & 2093 & 0.9 \\ 36 & 17825 & 1647.56 & 4.1176 & 5567 & 2.3 \\ 37 & 17843 & 1643.16 & 4.1066 & 4856 & 2.0 \\ 38 & 17851 & 1641.20 & 4.1017 & 5482 & 2.3 \\ 39 & 17881 & 1633.87 & 4.0833 & 1696 & 0.7 \\ 40 & 18495 & 1483.72 & 3.7081 & 2862 & 1.2 \\ 41 & 18567 & 1466.12 & 3.6641 & 1643 & 0.7 \\ 42 & 18606 & 1456.58 & 3.6403 & 2031 & 0.8 \\ 43 & 18797 & 1409.88 & 3.5235 & 4095 & 1.7 \\ 44 & 18857 & 1395.20 & 3.4869 & 2432 & 1.0 \\ 45 & 18877 & 1390.31 & 3.4747 & 1864 & 0.8 \\ 46 & 18884 & 1388.60 & 3.4704 & 2034 & 0.8 \\ 48 & 18911 & 1382.00 & 3.4539 & 1722 & 0.7 \\ & 18946 & 1373.44 & 3.4325 & 2187 & 0.9\end{array}$

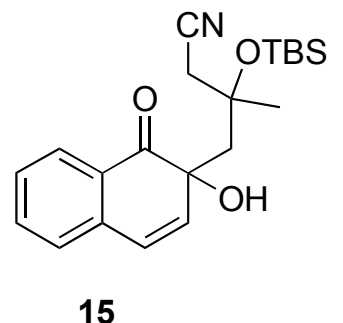




\begin{tabular}{|c|c|c|c|c|c|}
\hline 49 & 18974 & 1366.59 & 3.4154 & 1082 & 0.4 \\
\hline 50 & 20001 & 1115.46 & 2.7877 & 2987 & 1.2 \\
\hline 51 & 20019 & 1111.06 & 2.7767 & 3239 & 1.3 \\
\hline 52 & 20068 & 1099.08 & 2.7468 & 8047 & 3.3 \\
\hline 53 & 20085 & 1094.92 & 2.7364 & 7654 & 3.2 \\
\hline 54 & 20134 & 1082.94 & 2.7065 & 8720 & 3.6 \\
\hline 55 & 20152 & 1078.54 & 2.6955 & 7973 & 3.3 \\
\hline 56 & 20278 & 1047.72 & 2.6185 & 9190 & 3.8 \\
\hline 57 & 20302 & 1041.86 & 2.6038 & 8873 & 3.7 \\
\hline 58 & 20340 & 1032.56 & 2.5806 & 6576 & 2.7 \\
\hline 59 & 20345 & 1031.34 & 2.5775 & 6350 & 2.6 \\
\hline 60 & 20362 & 1027.18 & 2.5671 & 6452 & 2.7 \\
\hline 61 & 20370 & 1025.23 & 2.5622 & 5891 & 2.4 \\
\hline 62 & 20408 & 1015.94 & 2.5390 & 3640 & 1.5 \\
\hline 63 & 20430 & 1010.56 & 2.5256 & 3566 & 1.5 \\
\hline 64 & 20998 & 871.66 & 2.1784 & 5779 & 2.4 \\
\hline 65 & 21029 & 864.08 & 2.1595 & 5677 & 2.3 \\
\hline 66 & 21058 & 856.99 & 2.1418 & 7456 & 3.1 \\
\hline 67 & 21090 & 849.16 & 2.1222 & 7519 & 3.1 \\
\hline 68 & 21110 & 844.27 & 2.1100 & 3439 & 1.4 \\
\hline 69 & 21131 & 839.14 & 2.0972 & 3271 & 1.4 \\
\hline 70 & 21169 & 829.85 & 2.0739 & 4339 & 1.8 \\
\hline 71 & 21189 & 824.96 & 2.0617 & 4167 & 1.7 \\
\hline 72 & 21234 & 813.95 & 2.0342 & 6177 & 2.6 \\
\hline 73 & 21300 & 797.81 & 1.9939 & 1593 & 0.7 \\
\hline 74 & 21442 & 763.09 & 1.9071 & 8024 & 3.3 \\
\hline 75 & 21455 & 759.91 & 1.8992 & 7395 & 3.1 \\
\hline 76 & 21502 & 748.42 & 1.8704 & 6170 & 2.5 \\
\hline 77 & 21516 & 744.99 & 1.8619 & 5608 & 2.3 \\
\hline 78 & 21589 & 727.14 & 1.8173 & 4094 & 1.7 \\
\hline 79 & 21618 & 720.05 & 1.7995 & 4063 & 1.7 \\
\hline 80 & 21647 & 712.96 & 1.7818 & 3405 & 1.4 \\
\hline 81 & 21677 & 705.62 & 1.7635 & 3391 & 1.4 \\
\hline 82 & 23158 & 343.47 & 0.8584 & 140333 & 58.0 \\
\hline 83 & 23216 & 329.29 & 0.8230 & 242069 & 100.0 \\
\hline 84 & 24394 & 41.23 & 0.1030 & 38594 & 15.9 \\
\hline 85 & 24458 & 25.58 & 0.0639 & 50226 & 20.7 \\
\hline 86 & 24475 & 21.42 & 0.0535 & 89023 & 36.8 \\
\hline 87 & 24661 & -24.06 & -0.0601 & 70120 & 29.0 \\
\hline
\end{tabular}

